\title{
Sixteenth
}

International

Kidney Cancer

Symposium

November 3-4, 2017

National Doral Miami, Miami, Florida

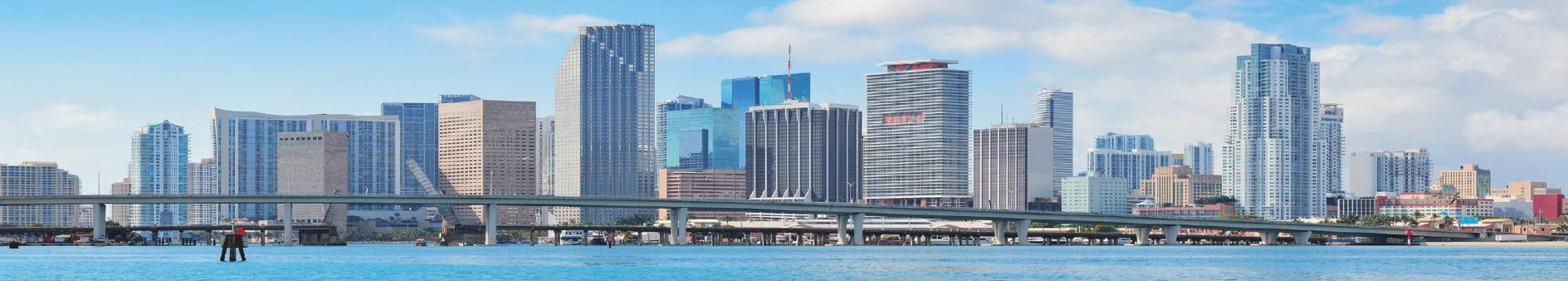

KidneyCancer.org

www.kidneycancersymposium.com

For more information about the Kidney Cancer Association and about the Sixteenth International Kidney Cancer Symposium in Miami go to:

www.kidneycancer.org

www.kidneycancersymposium.com 
Supplement

\section{Abstracts from the Sixteenth International Kidney Cancer Symposium, 2nd-3rd November 2017, Miami, Florida}

\section{CONTENTS}

01 A Phase 1b/2 Trial of Lenvatinib+Pembrolizumab in Patients With Renal Cell Carcinoma..........S1

02 A Phase 2 Trial of Lenvatinib $18 \mathrm{mg}$ vs $14 \mathrm{mg}$ Once Daily (QD) in Combination With

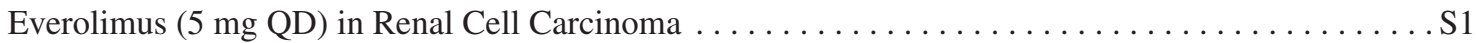

03 A Phase 3 Trial to Compare Efficacy and Safety of Lenvatinib in Combination With Everolimus or Pembrolizumab vs Sunitinib Alone in First-line Treatment of Patients With Metastatic

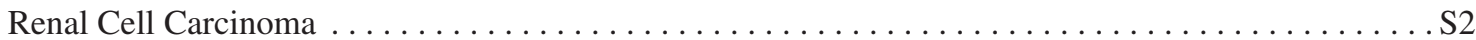

04 A phase I, open label, dose escalation and cohort expansion study to evaluate the safety and immune response to autologous dendritic cells transduced with AdGMCA9 in patients with metastatic renal cell carcinoma. . . . . . . . . . . . . . . . . . . . . . . . S3

05 A Phase II Study of the Efficacy and Safety of Axitinib (Axi) Given on an Individualized Schedule for metastatic renal cell carcinoma (mRCC) after treatment with PD-1 / PD-L1

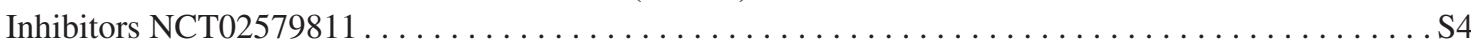

06 Adjuvant sunitinib (SU) in patients (pts) with high risk renal cell carcinoma (RCC): Safety

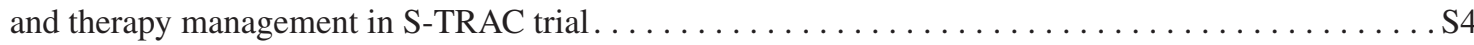

07 Association between pretreatment neutrophil-to-lymphocyte ratio and outcome of patients with metastatic renal cell carcinoma treated with nivolumab $\ldots \ldots \ldots \ldots \ldots \ldots \ldots \ldots \ldots \ldots \ldots$

08 Association between stool bacteriomic profile and response to sunitinib in metastatic renal

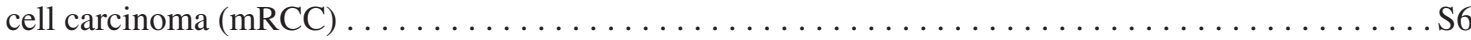

09 Axitinib and Cabozantinib in the treatment of sunitinib-refractory patients with metastatic renal cell carcinoma (mRCC): Results of matching adjusted indirect treatment comparison (MAIC)

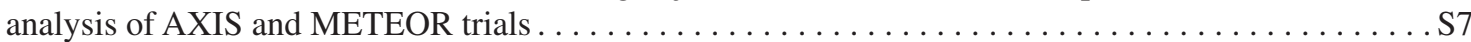

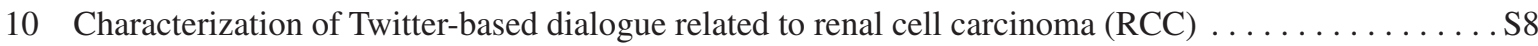

11 Checkpoint Inhibitors in the Management of Renal Cell Carcinoma with Sarcomatoid Features .......S9

12 Clinical Activity of Nivolumab in Patients with Non-Clear Cell Renal Cell Carcinoma . . . . . . . . . . S9

13 Clinical outcome of patients with metastatic Renal Cell Carcinoma (mRCC) progressing on front-line combination regimens that include checkpoint inhibitors $\ldots \ldots \ldots \ldots \ldots \ldots \ldots$. 10

14 Clinical outcomes of patients treated with local therapies with oligometastatic renal

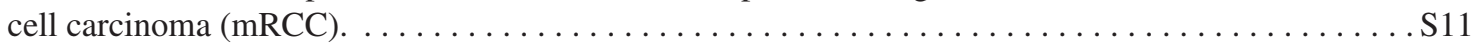

15 Clinicopathological characterization and oncologic outcomes of metastatic small renal masses......S12

16 Comparative Genomic Profiling of Matched Primary and Metastatic Tumors

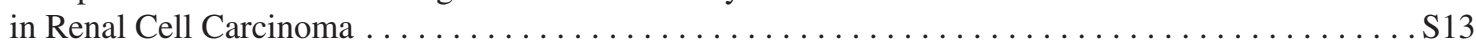

17 DART Study: A phase 2 randomized trial of dalantercept plus axitinib versus placebo plus axitinib in advanced renal cell carcinoma (RCC): Results from the part 2 placebo-controlled trial. . . S14

18 Deferred Systemic Therapy (DST) for Metastatic Renal Cell Carcinoma: Preliminary

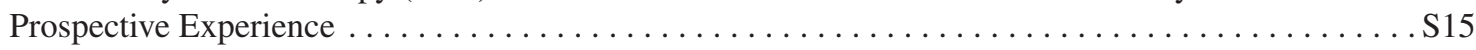

19 Do CT perfusion measures differ in primary renal tumors versus metastatic lesions in patients

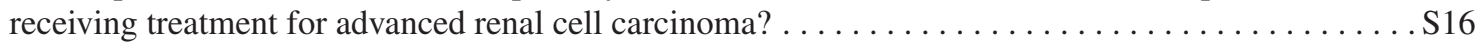


20 Effects of pazopanib (PAZ) and sunitinib (SUN) dose modification on safety and efficacy in patients with metastatic renal cell carcinoma $(\mathrm{mRCC})$ from COMPARZ . . . . . . . . . . S18

21 Estimating the Social Value Generated by Immunotherapy for Renal Cell Carcinoma Patients. . . . . . S18

22 Genomic Heterogeneity and the Small Renal Mass . . . . . . . . . . . . . . . . . . . . . . S19

23 Germline Mutations in Cancer-Susceptibility Genes in Patients with Advanced

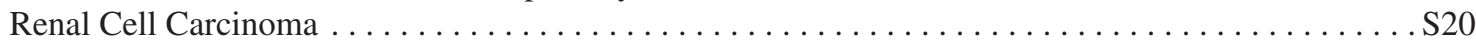

24 IMmotion150: Novel Radiological Endpoints and Updated Data From a Randomized Phase II Trial Investigating Atezolizumab With or Without Bevacizumab vs Sunitinib

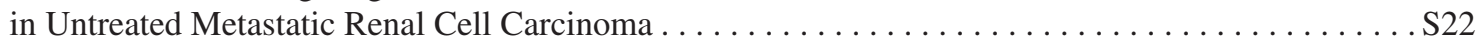

25 Immune Cell Infiltration within Differing Renal Cell Carcinoma Primary Histologies: Preliminary Report. . . . . . . . . . . . . . . . . 23

26 Impact of antibiotics on outcome in patients with metastatic renal cell carcinoma treated

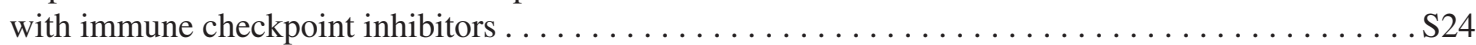

27 Integrated biomarker analysis for 412 renal cell cancer (RCC) patients (pts) treated on the phase 3 COMPARZ trial: Correlating common mutation events in PBRM1 and BAP1 with angiogenesis expression signatures and outcomes on tyrosine kinase inhibitor (TKI) . . . . . S25

28 Long-term response and time to response to pazopanib (PAZ) and sunitinib (SUN) in metastatic

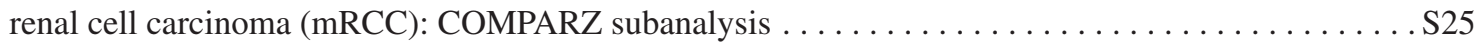

29 Physician Treatment Selection in the Prospective Metastatic Renal Cell Cancer (MaRCC) Registry . . S26

30 Plasma glycosaminoglycan scores in early stage renal cell carcinoma . . . . . . . . . . . S27

31 Predictive genomic markers of response to VEGF targeted therapy (TT) in metastatic renal cell carcinoma (mRCC): Role of VHL and TP53 mutation, and FLT1 germline variant . . . . . . . S28

32 Productivity, Satisfaction, and Health-Related Quality of Life in Advanced Renal Cell Carcinoma Patients Receiving 2 or More Lines of Treatment: Results from a United

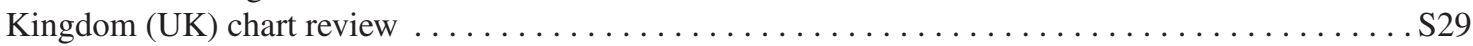

33 PT2977, a Novel HIF-2a Antagonist, Affords Potent Anti-Tumor Activity and Remodels the Immunosuppressive Tumor Microenvironment in Clear Cell Renal Cell Cancer . . . . . . . . . . . . . S29

34 Quality-adjusted survival of nivolumab vs. everolimus in patients with previously treated

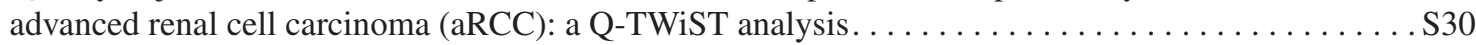

35 Results of lymph node dissection for locally advanced and metastatic renal cell carcinoma . . . . . . S31

36 Rheumatologic adverse events in patients with metastatic renal cell carcinoma treated with immune checkpoint inhibitors . . . . . . . . . . . . . . . . . . . . 31

37 Safety of Nivolumab in Patients With Clear Cell (CC) or Non-Clear Cell (NCC) Renal Cell Carcinoma (RCC): Results From the Phase IIIb/IV CheckMate 374 Study . . . . . . . . . . . S32

38 Savolitinib versus sunitinib in patients with MET-driven, unresectable and locally advanced or metastatic papillary renal cell carcinoma: SAVOIR, a randomised, phase III trial . . . . . . . S33

39 Second-Line Treatment of Metastatic Renal Cell Carcinoma: Systematic Review

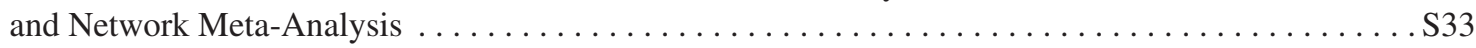

40 Systemic therapy for oligo-progressive, metastatic renal cell carcinoma ( $\mathrm{mRCC}$ ) treated

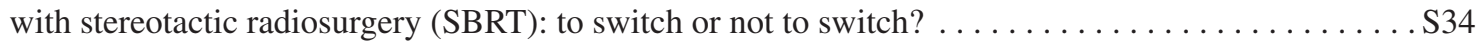

41 The Association between Insurance Status and Survival in Metastatic Renal Cell Carcinoma

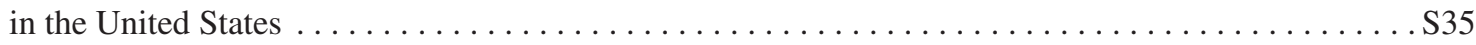


42 The association of sarcopenia and tumor aggressiveness in clear cell renal cell carcinoma . . . . . . S40

43 The impact of bone metastasis location in the clinical outcome of patients with metastatic renal cell carcinoma (mRCC): an analysis from the Latin American Renal Cancer Group (LARCG) . . . . . S40

44 Three-Year Efficacy and Safety Update From the Phase III CheckMate 025 Study of Nivolumab Versus Everolimus in Patients With Advanced Renal Cell Carcinoma (aRCC) . . . . . . . . . S4

45 TiNiVo: A Phase Ib Dose Escalation Trial of Tivozanib and Nivolumab in Renal Cell Carcinoma . . . S42

46 Treatment of metastatic renal cell carcinoma with the mushroom toxin orellanine . . . . . . . . . S43

47 Treatment patterns among patients with metastatic renal cell carcinoma receiving systemic therapies in US real-world settings between 2006 and $2017 \ldots \ldots \ldots \ldots \ldots \ldots \ldots \ldots \ldots \ldots \ldots$

48 Updated Results From a Phase I Study of Nivolumab in Combination With Ipilimumab in Metastatic Renal Cell Carcinoma: The CheckMate 016 Study . . . . . . . . . . . . . . . . . . . S44

49 Updated Results From a Phase I Study of Nivolumab in Combination With Sunitinib or Pazopanib in Metastatic Renal Cell Carcinoma: The CheckMate 016 Study . . . . . . . . . . . S45

50 Validation of the Preoperative Nomogram Predicting 12-Year Probability of Metastatic Renal Cancer . . . . . . . . . . . . . . . . . . . . . . . . . . . . . . . . . S46

51 Variations in treatment patterns for metastatic renal cell carcinoma (mRCC) between

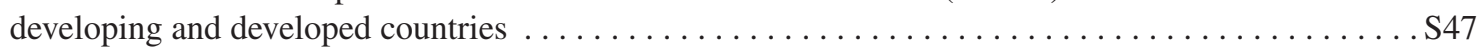

52 Active Surveillance for von Hippel-Lindau-Related Renal Tumors using Size-Based Risk Stratification: Long-term Results. 
01

\section{A Phase 1b/2 Trial of Lenvatinib+Pembrolizumab in Patients With Renal Cell Carcinoma}

Lee, Chung-Han (Memorial Sloan-Kettering Cancer Center, New York, United States); Makker, Vicky (Memorial Sloan-Kettering Cancer Center, New York, United States); Rasco, Drew (START, San Antonio, TX, United States); Taylor, Matthew (Knight Cancer Institute, Portland, OR, United States); Dutcus, Corina; Shumaker, Robert (Eisai Inc., Woodcliff Lake, NJ, United States); Schmidt, Emmett V. (Merck \& Co., Inc., Kenilworth, NJ, United States); Stepan, Daniel E.; Li, Di (Eisai Inc., Woodcliff Lake, NJ, United States); Motzer, Robert (Memorial Sloan-Kettering Cancer Center, New York, NY, United States)

Introduction/objective: Lenvatinib (LEN) is a multikinase inhibitor of vascular endothelial growth factor (VEGF) receptor 1-3, fibroblast growth factor receptor 1-4, platelet-derived growth factor receptor alpha, RET, and KIT. LEN was approved in combination with everolimus to treat advanced renal cell carcinoma (RCC) after 1 prior VEGF-targeted treatment. We report results for the RCC cohort of a phase $1 \mathrm{~b} / 2$ trial of LEN+pembrolizumab (PEM) in patients with selected solid tumors (NCT02501096).

Methods: This was a multicenter open-label study. Patients had metastatic clear cell RCC, measurable disease according to immune-related Response Evaluation Criteria in Solid Tumors (irRECIST), and Eastern Cooperative Oncology Group performance status $\leq 1$. LEN $20 \mathrm{mg} / \mathrm{d}$ plus PEM $200 \mathrm{mg}$ intravenously every 3 weeks was assessed as the maximum tolerated dose and recommended phase 2 dose in phase $1 \mathrm{~b}$. Tumor assessments were performed by trial investigators using irRECIST. The primary phase 2 endpoint was objective response

\begin{tabular}{|c|c|c|}
\hline Outcome & $\mathbf{n}=\mathbf{3 0}$ & $95 \% \mathrm{CI}$ \\
\hline Objective response rate, $\mathrm{n}(\%)$ & $19(63.3)$ & $43.9 \%-80.1 \%$ \\
\hline $\begin{array}{l}\text { Median progression-free } \\
\text { survival, months }\end{array}$ & NE & 9.9-NE \\
\hline $\begin{array}{l}\text { Median duration of response, } \\
\text { months } \\
\text { NE, not estimable. }\end{array}$ & $\mathrm{NE}$ & 8.4-NE \\
\hline
\end{tabular}

rate at 24 weeks. Secondary endpoints included objective response rate, progression-free survival, and duration of response.

Results: 30 Patients were enrolled in either the phase $1 \mathrm{~b}$ ( 8 patients) or phase 2 cohort (22 patients). Data cutoff was March 1, 2017. 12 (40\%) Patients had $0,10(33 \%)$ patients had 1 , and $8(27 \%)$ patients had $\geq 2$ prior anti-cancer therapies. Of patients who received prior medication $(\mathrm{n}=18[60 \%]), 16(53 \%)$ received prior VEGF-targeted therapy. Efficacy outcomes are summarized in the table. At data cutoff, $17(57 \%)$ patients were still receiving treatment, $8(27 \%)$ completed treatment due to disease progression, and $5(17 \%)$ discontinued treatment. The most common any-grade treatment-emergent adverse events were diarrhea $(83 \%)$, fatigue $(70 \%)$, hypothyroidism $(67 \%)$, stomatitis $(60 \%)$, hypertension $(57 \%)$, and nausea $(57 \%)$. Toxicities were manageable with dose interruption and/or modification and no new safety signals were found.

Conclusions: Combination treatment with LEN+PEM showed promising antitumor activity and an acceptable safety profile. A phase 3 trial of LEN+PEM and LEN+everolimus, vs sunitinib in first-line treatment for metastatic clear cell RCC is ongoing.

02

\section{A Phase 2 Trial of Lenvatinib $18 \mathrm{mg}$ vs 14 mg Once Daily (QD) in Combination With Everolimus (5 mg QD) in Renal Cell Carcinoma}

Pal, Sumanta K (City of Hope, Duarte, CA United States); Puente, Javier (Hospital Clinico Universitario, Madrid, Spain); Heng, Daniel (Tom Baker Cancer Center, Calgary, AB, Canada); Rha, Sun Young (Yonsei Cancer Center, Yonsei University College of Medicine, Seoul, Korea, Republic of (South)); Li, Di (Eisai Inc., Woodcliff Lake, NJ, United States); Stepan, Daniel E; Dutcus, Corina E (Eisai Inc, Woodcliff Lake, NJ, United States); Glen, Hilary (Beatson West of Scotland Cancer Centre, Glasgow, SC, United Kingdom)

Introduction/objective: Based on findings from a phase 2 study, lenvatinib (LEN) plus everolimus 
(EVE) was approved in the United States and European Union for patients with advanced renal cell carcinoma (RCC) following 1 prior antiangiogenic therapy. In that study, LEN $18 \mathrm{mg}$ QD plus EVE $5 \mathrm{mg}$ QD significantly prolonged progression-free survival (PFS) compared with either monotherapy. In the LEN+EVE cohort, grades 3 and 4 treatment-emergent adverse events (TEAEs) occurred in $71 \%$ of patients. We report the design of a multicenter, randomized, double-blind, phase 2 study to evaluate if a lower LEN starting dosage regimen provides similar efficacy with a better safety profile than LEN $18 \mathrm{mg}$ plus EVE $5 \mathrm{mg}$ (NCT03173560).

Methods: Eligible patients are aged $\geq 18$ years with histologic or cytologic confirmation of predominantly clear cell RCC, advanced RCC, 1 prior anti-vascular endothelial growth factor therapy for advanced $\mathrm{RCC}, \geq 1$ measurable target lesion according to RECIST 1.1, and a Karnofsky Performance Status score of $\geq 70$. Patients will receive LEN $18 \mathrm{mg}$ or $14 \mathrm{mg}$ QD plus EVE $5 \mathrm{mg}$ QD in 28-day cycles until disease progression, unacceptable toxicity, or withdrawal of consent. The LEN 14-mg dose will be escalated to $18 \mathrm{mg}$ if no intolerable grade 2 , or any grade $\geq 3$, TEAEs requiring dose reduction occur in cycle 1 . The primary endpoints are objective response rate $(\mathrm{ORR})$ at week $24\left(\mathrm{ORR}_{24 \mathrm{~W}}\right)$ and the proportion of patients with intolerable grade 2, and any grade $\geq 3$, TEAEs within 24 weeks after randomization. Secondary endpoints include PFS and ORR. An estimated 306 patients will be randomized. Sample size is based on detecting noninferiority of $\mathrm{ORR}_{24 \mathrm{~W}}$ and superiority of the primary safety endpoint. Two interim analyses will be performed when 150 and 200 patients have completed 24 weeks of follow-up or discontinue earlier. Each analysis will test noninferiority and futility of the LEN 14-mg arm $\mathrm{ORR}_{24 \mathrm{~W}}$ versus the 18 -mg arm $\mathrm{ORR}_{24 \mathrm{w}}$. An O'Brien-Fleming boundary will be used for noninferiority. If the 1 -sided $P$-value is $\leq 0.005$ at the first interim analysis, $\leq 0.014$ at the second interim analysis, or $\leq 0.045$ at the final analysis, then noninferiority in $\mathrm{ORR}_{24 \mathrm{~W}}$ will be claimed. If the futility boundary is crossed (ie, 1 -sided $P$-value is $\geq 0.776$ at the first interim analysis or $\geq 0.207$ at the second interim analysis), then futility will be claimed.

\section{A Phase 3 Trial to Compare Efficacy and Safety of Lenvatinib in Combination With Everolimus or Pembrolizumab vs Sunitinib Alone in First-line Treatment of Patients With Metastatic Renal Cell Carcinoma}

Motzer, Robert (Memorial Sloan-Kettering Cancer Center, New York United States); Grünwald, Viktor (Hannover Medical School, Niedersachsen, Hannover, Germany); Hutson, Thomas E (Baylor University Medical Center, Dallas, United States); Porta, Camillo (IRCCS San Matteo University Hospital Foundation, Pavia, Italy); Powles, Thomas (Barts Cancer Institute, London, United Kingdom); Eto, Masatoshi (Kyushu University, Fukuoka, Japan); Dutcus, Corina E; Baig, Mahadi A; Dutta, Lea; Li, Di (Eisai Inc., Woodcliff Lake, United States); Choueiri, Toni K (Dana Farber Cancer Institute, Boston, United States)

Introduction/objective: Lenvatinib (LEN) is a multikinase inhibitor of vascular endothelial growth factor (VEGF) receptor 1-3, fibroblast growth factor receptor 1-4, platelet-derived growth factor receptor alpha, RET and KIT. Based on a phase 2 study (Motzer et al. Lancet Oncol. 2015), LEN was approved in combination with everolimus (EVE) for the treatment of metastatic renal cell carcinoma (RCC) following 1 prior VEGF-targeted therapy. A phase $1 \mathrm{~b} / 2$ study of LEN in combination with pembrolizumab (PEM) in patients with RCC LEN is also underway. We report the design of a multicenter, open-label, phase 3 trial of LEN plus EVE or PEM vs sunitinib (SUN; a standard therapy for RCC) as first-line treatment for advanced RCC (NCT02811861).

Methods: Patients aged $\geq 18$ years with confirmed advanced RCC diagnosis, $\geq 1$ measurable lesion per Response Evaluation Criteria in Solid Tumors (RECIST) v1.1, Karnofsky Performance Status $\geq 70$, controlled blood pressure, and adequate blood coagulation, renal, hepatic, and bone marrow function are eligible. Patients will be randomized 1:1:1 to receive LEN $18 \mathrm{mg} / \mathrm{d}$ + EVE $5 \mathrm{mg} / \mathrm{d}$, LEN $20 \mathrm{mg} / \mathrm{d}+$ PEM $200 \mathrm{mg}$ every 3 weeks, or SUN $50 \mathrm{mg} / \mathrm{d}$ (on a schedule of 4 weeks on treatment followed by 2 weeks off) until disease progression, unacceptable toxicity, withdrawal of consent, or study end. The primary endpoint is to show superiority of LEN+EVE or LEN+PEM over single-agent SUN as 
first-line treatment for advanced RCC in improving progression-free survival (PFS). Secondary endpoints include comparison of objective response rate, overall survival, PFS on next-line therapy, health-related quality of life, and safety and tolerability in patients receiving LEN+EVE or LEN+PEM vs SUN. Exploratory endpoints include PFS in the LEN+PEM arm using immune-related RECIST, comparison of duration of response, disease control rate, and clinical benefit rate in patients treated with LEN+EVE or LEN+PEM vs SUN, and analysis of the relationship between blood biomarkers and outcome. No interim analysis is planned for efficacy or futility. Enrollment of 735 patients is planned to achieve $90 \%$ power at 2 -sided $\alpha=0.05$ to detect a difference in $\geq 1$ of the primary comparisons.

\section{4}

\section{A phase I, open label, dose escalation and cohort expansion study to evaluate the safety and immune response to autologous dendritic cells transduced with AdGMCA9 in patients with metastatic renal cell carcinoma}

Faiena, Izak (UCLA, Los Angeles, CA, United States); Zomorodian, Nazy (UCLA, David Geffen School of Medicine, Los Angeles, CA, United States); CominAnduix, Begoña; Sachdeva, Ankush; Bot, Adrian; Kabinnavar, Fairouz; Said, Jonathan (UCLA, Los Angeles, $C A$, United States); Cheung-Lau, Gardenia (UCLA, United States); Macabali, Mignonette (UCLA, Los Angeles, CA, United States); Cabrera, Paula (UCLA, Mexico, DF, Mexico); Kaplan-Lefko, Paula; BerentMaoz, Beata (UCLA, Los Angeles, CA, United States); Pantuck, Allan J. (University of California, Los Angeles, Los Angeles, CA, United States); Belldegrun, Arie S.; Drakaki, Alexandra (UCLA, Los Angeles, CA, United States)

Introduction: Ubiquitous membranous expression of carbonic anhydrase IX (CAIX) in renal cell carcinoma (RCC) makes it an attractive vaccine target. We developed a fusion gene construct, GM-CSF + CAIX, transduced by a replication deficient adenovirus into autologous dendritic cells (DC) that are injected in patients with metastatic RCC in this phase 1 study targeting CAIX overexpressed on RCC tumors.
Methods: A recombinant adenovirus encoding the GMCSF-CAIX fusion gene (AdGMCAIX) was manufactured per GMP in collaboration with the NCI Rapid Access to Intervention Development (RAID) program. The final product was produced using DCs cultured ex vivo from patients' peripheral blood mononuclear cells (PBMC) and engineered with AdGMCAIX prior to intradermal injection. These injected transduced DCs were expected to stimulate an antigen specific immune response against CAIX expressing RCC. Three dose escalation cohorts $\left(5,15\right.$ and $50 \times 10^{6}$ cells/administration) were injected based on $3+3$ design. DC-AdGMCAIX was given intradermally Q2 weeks $\mathrm{X} 3$ doses. The primary aim is safety of the injections. Secondary aims are to evaluate immune responses \& antitumor effects per RECIST 1.1. Eligibility criteria included patients with clear cell mRCC with ECOG 0-1, measurable disease, and adequate organ function.

Results: Fifteen patients with clear cell $\mathrm{mRCC}$ were enrolled. Nine patients received all 3 planned vaccine doses. No SAE's were seen. Grade 1/2 AEs include fatigue (3/1), leukopenia (1/1) and flu-like symptoms $(0 / 1)$. Of the nine patients who received treatment, one expired of progressive disease, two patients were lost to follow-up and six patients are alive. Of the six patients, five have progressive disease and are currently receiving standard-of-care therapies, and one has completed treatment with stable disease at 6 mon follow up and is being evaluated for retreatment.

Conclusions: These early data show that autologous DC transduced by Ad-GMCAIX vector can be safely given to $\mathrm{mRCC}$ patients without any SAE's noted at the doses tested. These data support further development of Ad-GMCAIX vaccine strategies either alone or in combination with approved therapies.

Funding: Supported by NCI RAID Initiative NSC 740833 and Kite Pharma 
05

\section{A Phase II Study of the Efficacy and Safety of Axitinib (Axi) Given on an Individualized Schedule for metastatic renal cell carcinoma ( $m R C C)$ after treatment with PD-1 / PD-L1 Inhibitors NCT02579811}

\begin{abstract}
Wood, Laura (Cleveland Clinic Cancer Center, Cleveland, $\mathrm{OH}$, United States); Allman, Kimberly; Ornstein, Moshe; Martin, Allison (Cleveland Clinic Cancer Center, Cleveland, OH, United States); Garcia, Jorge (9500 Euclid Ave CA-6, Cleveland, OH, United States); Gilligan, Timothy; Grivas, Petros; Company, Donna (Cleveland Clinic Cancer Center, Cleveland, $\mathrm{OH}$, United States); Olencki, Thomas (The Ohio State University Wexner Medical Center, Columbus, $\mathrm{OH}$, United States); Sumanta, Pal (City of Hope Cancer Center, Duarte, CA, United States); Rathmell, Wendy (Vanderbilt-Ingram Cancer Center, Nashville, TN, United States); Rini, Brian (Cleveland Clinic Cancer Center, Cleveland, $\mathrm{OH}$, United States)
\end{abstract}

Background: Axitinib is a vascular endothelial growth factor receptor (VEGFR) tyrosine kinase inhibitor (TKI) approved for the treatment of $\mathrm{mRCC}$ after failure of 1 systemic therapy. Pharmacokinetics (PKs) demonstrate significant inter-patient variability, and clinical data indicate that higher exposure is associated with improved clinical outcomes. ${ }^{1,2}$ The current-recommended Axi titration from $5 \mathrm{mg}$ to $7 \mathrm{mg}$ to $10 \mathrm{mg}$ BID is often not tolerated by many patients (pts). As such, many pts do not undergo dose-titration resulting in lower than necessary drug plasma levels. Further, no prospective data exists on the efficacy of Axi in the post-PD-1/PD-L1 setting. This study aims to identify a more individualized dosetitration algorithm and to prospectively assess the clinical efficacy of Axi after PD-1/PD-L1 inhibition.

Methods: Eligibility criteria include clear cell $\mathrm{mRCC}$ following progression on PD-1/PD-L1 therapy, measurable disease, and adequate organ function. Pts will be treated with Axi 5mg BID, with dose titration in $1 \mathrm{mg}$ increments every 14 days if no grade (G) 2 Axi-related mucositis, diarrhea, hand-footsyndrome, or fatigue (other toxicities are not considered). Instead of dose reduction for $\mathrm{G} 2$ adverse events (AEs), pts will have a brief break (i.e. 3 days per physician discretion), then resume the same dose if AE becomes G1 or less. Dose reduction in $1 \mathrm{mg}$ increments will be done for recurrent G2 AEs in spite of treatment break, and per physician discre- tion. The intent is to rapidly titrate Axi with smaller dosing increments and utilize occasional, brief breaks in order to maximize dose intensity with tolerable AEs. Response will be assessed by standard imaging studies every 8 weeks. To date, 24/50 pts have been enrolled, with the goal of 44 evaluable pts to test the hypothesis that individualized dose titration will lead to $40-45 \%$ increase in median PFS (from 7 to 10 months) in the post PD-1 / PD-L1 inhibitor setting.

\section{References}

[1] Klumpen HJ, Samer CF, Mathijssen RH, et al. Moving towards dose individualization of tyrosine kinase inhibitors. Cancer Treat Rev 2011;37:251-260.

[2] Rini BI, Garrett M, Poland B, et al. Axitinib in metastatic renal cell carcinoma: Results of a pharmacokinetic and pharmacodynamics analysis. $J$ Clin Pharmacol 2013;53:491-504.

\section{6}

\section{Adjuvant sunitinib (SU) in patients (pts) with high risk renal cell carcinoma (RCC): Safety and therapy management in S-TRAC trial}

Daniel J George ${ }^{1}$, Robert J Motzer ${ }^{2}$, Michael Staehler ${ }^{3}$, Hardev S Pandha ${ }^{4}$, Frede Donskov ${ }^{5}$, Bernard Escudier ${ }^{6}$, Jan Kliment ${ }^{7}$, Allan J Pantuck ${ }^{8}$, Anup Patel ${ }^{9}$, Liza DeAnnuntis ${ }^{10}$, Helen Bhattacharyya ${ }^{11}$, Xun Lin ${ }^{12}$, Mariajose Lechuga ${ }^{13}$, Lucile Serfass ${ }^{14}$, Jean-Jacques Patard $^{15}$, Alain Ravaud ${ }^{16}$

${ }^{1}$ Duke Cancer Center, Division of Oncology, Durham, NC, USA;

${ }^{2}$ Memorial Sloan Kettering Cancer Center, Department of Oncology, New York, NY, USA,

${ }^{3}$ University Hospital of Munich, Department of Urology, Munich, Germany;

${ }^{4}$ Department of Clinical and Experimental Medicine, University of Surrey, Department of Microbial Sciences, Surrey, UK;

${ }^{5}$ Department of Oncology, Aarhus University Hospital, Aarhus, Denmark;

${ }^{6}$ Institut Gustave Roussy, Department of Medical

Oncology, Villejuif, France,

${ }^{7}$ Department of Urology, University Hospital, Martin, Slovakia;

${ }^{8}$ Department of Urology, Ronald Reagan UCLA Medical Center, Los Angeles, CA, USA,

${ }^{9}$ Spire Roding Hospital, London, UK;

${ }^{10}$ Pfizer Inc, Collegeville, PA, USA;

${ }^{11}$ Pfizer Inc, New York, NY, USA;

${ }^{12}$ Pfizer Inc, La Jolla, CA, USA;

${ }^{13}$ Pfizer S.r.L, Milan, Italy, 
Table. Most common AEs leading to TDC, dose RED and INT*

\begin{tabular}{|c|c|c|c|c|c|c|c|c|}
\hline \multicolumn{3}{|l|}{ Treatment DC } & \multicolumn{3}{|l|}{ Dose RED } & \multicolumn{3}{|l|}{ Dose INT } \\
\hline $\mathrm{AE}, \%$ & SU & PBO & $\mathrm{AE}, \%$ & SU & PBO & $\mathrm{AE}, \%$ & SU & PBO \\
\hline PPE & 4.2 & 0 & PPE & 11.8 & 0.7 & PPE & 6.2 & 0 \\
\hline Hypertension & 2.0 & 0 & Fatigue & 3.9 & 0.3 & Hypertension & 5.6 & 0 \\
\hline Asthenia & 1.3 & 0 & Diarrhoea & 2.6 & 0 & Neutropenia & 5.2 & 0 \\
\hline Fatigue & 1.0 & 0.3 & Mucosal inflammation & 2.6 & 0 & Nausea & 4.9 & 1.0 \\
\hline Pulmonary embolism & 1.0 & 0.3 & Neutropenia & 2.6 & 0 & Diarrhoea & 4.6 & 1.3 \\
\hline
\end{tabular}

*Many of the AEs leading to DC and INT were grade $1 / 2$

$\mathrm{TDC}=$ treatment discontinuation; $\mathrm{RED}=$ reduction; $\mathrm{INT}=$ interruption; $\mathrm{PPE}=$ palmar-plantar erythrodysesthesia syndrome.

${ }^{14}$ Pfizer Oncology, Paris, France;

${ }^{15}$ Centre Hospitalier De Mont De Marsan, Mont-de-

Marsan, France;

${ }^{16}$ Department of Medical Oncology, Bordeaux University Hospital, Bordeaux, France.

Background: Pts with locoregional RCC at high risk ( $\geq \mathrm{T} 3$ and/or $\mathrm{N}+$ ) of tumour recurrence post nephrectomy treated with adjuvant SU (50 mg daily; schedule 4/2) had significantly longer disease-free survival (DFS) vs. placebo (PBO; HR, 0.76; 95\% CI, $0.59-0.98 ; P=0.03)$. We report safety and therapy management data.

Methods: Reasons for SU treatment discontinuation (TDC), dose reduction (RED), dose interruption (INT), and pts TDC due to AEs by cycle, were summarized. Median time to SU TDC was calculated.

Results: Of the 615 pts enrolled, 306 were treated with SU at a median (range) daily dose of 45.9 (8.952.6) $\mathrm{mg}$. $71 \%$ of pts remained on SU treatment for $\geq 8$ months (mo) and 56\% completed the full 1-year treatment. Most common reasons for TDC were AEs $(28.1 \%)$ in SU arm, and relapse (19.4\%) in PBO arm. Common AEs leading to TDC, RED and INT are summarized in the Table. TDC due to AEs in cycles $1,3,6$, and 9 , respectively: $7.8 \%, 3.3 \%, 2.6 \%$, and $1.6 \%$ in SU arm, and $0.3 \%, 1.3 \%, 0.3 \%$, and $0 \%$ in PBO arm. In the 86 pts who DC SU, median time to TDC was 4.5 mo. Median time to first RED and INT in SU-treated pts was 2.9 and 3.0 mo, respectively. More data, including time on RED/INT, time to onset of common AEs and maximum severity and reversibility of AEs leading to permanent discontinuation will be presented.

Conclusions: No new safety signals were identified with sunitinib use in the adjuvant RCC setting. Effective therapy management, including dose RED/ INT if necessary, is important as it optimizes the possibility of receiving effective treatment.

Clinicaltrials.gov identifier: NCT00375674
07

\section{Association between pretreatment neutrophil-to-lymphocyte ratio and outcome of patients with metastatic renal cell carcinoma treated with nivolumab}

Dutcher, Giselle (Emory University, Atlanta, GA, United States); Liu, Yuan (Department of Biostatistics and Bioinformatics, Atlanta, GA, United States); Ravindranathan, Deepak ( $J$ Willis Hurst Internal Medicine Residency Program, Atlanta, GA, United States); Carthon, Bradley (Winship Cancer Institute of Emory University, Atlanta, GA, United States); Kissick, Haydn (Department of Urology, Emory University, Atlanta, GA, United States); Harris, Wayne; Kucuk, Omer (Winship Cancer Institute of Emory University, Atlanta, GA, United States); Master, Viraj (Department of Urology, Emory University, Atlanta, GA, United States); Bilen, Mehmet (Winship Cancer Institute of Emory University, Atlanta, GA, United States)

Background: Biomarkers to guide treatment in metastatic renal cell carcinoma (mRCC) are lacking. Existing literature shows that neutrophil-to-lymphocyte ratio (NLR) predicts prognosis for $\mathrm{mRCC}$ patients receiving targeted therapy. We aimed to investigate the association between pretreatment NLR and prediction of outcome in patients with $\mathrm{mRCC}$ receiving nivolumab.

Methods: We performed a retrospective chart review of 38 patients with $\mathrm{mRCC}$ treated with nivolumab as standard of care between 2015 - 2016 at Winship Cancer Institute of Emory University. NLR was determined from complete blood count collected prior to starting treatment and imaging was performed to assess progression. We defined clinical benefit as complete response (CR) or partial response (PR) or stable disease (SD) of greater than 4 months. Progression-free survival (PFS) was defined as time from nivolumab initiation to date of 
progression, hospice referral, or death from any cause. Overall survival (OS) was defined as time from nivolumab initiation to death or hospice referral. The NLR cutoff value of 5.5 was determined by $\log$ rank test, and the univariate association with OS or PFS was assessed by Cox proportional hazard model and Kaplan-Meier method.

Results: The 38 patients had a median age of 68.5 years; 29 (76\%) were men and 9 (24\%) were women. Within the cohort, tumor histology includes 20 (53\%) non-clear cell and $18(47 \%)$ clear cell. The majority of patients (45\%) had KPS score of 80-100. MSKCC score within the cohort showed 4 (10\%) patients with good risk, $20(53 \%)$ patients with intermediate risk, and 14 (37\%) with poor risk. Response was evaluable for 32 patients; the other six patients did not complete two cycles ( 8 weeks) of treatment. One patient experienced CR, one patient had PR, and 15 patients (40\%) experienced SD. Among those 15 patients with SD, 12 patients $(80 \%)$ had stable disease for greater than 4 months. In our cohort, a total of 17 patients $(53 \%)$ had clinical benefit after nivolumab treatment. The PFS and OS for all patients at 12 months was $54 \%$ and $69 \%$, respectively. The median PFS was 2.6 months in the high NLR group but not reached in the low NLR group (Figure 1A). Low NLR was strongly associated with increased PFS with hazard ratio HR of 0.26 (95\% CI 0.09-0.74; $p=0.012$ ). The median OS was 2.7 months in the high NLR group but not reached in the low NLR group (Figure 1B). Again, low NLR was

Figure 1A. Kaplan-Meier Curve of Progression Free Survival

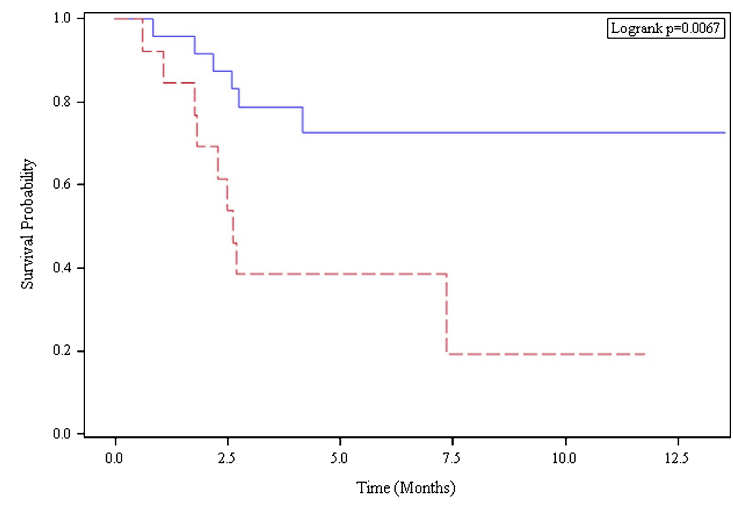

Baseline Neutrophil-Lymphocyte Ratio (C) $\quad<=5.5--->5.5$

\begin{tabular}{lcc}
\hline $\begin{array}{l}\text { Baseline Neutrophil- } \\
\text { Lymphocyte-Ratio }(\mathbf{C})\end{array}$ & $\begin{array}{c}\text { Number of } \\
\text { Subjects }\end{array}$ & $\begin{array}{c}\text { Median Survival } \\
\mathbf{( 9 5 \%} \mathbf{C I})\end{array}$ \\
\hline$<=5.5$ & 25 & NA $(4.2, \mathrm{NA})$ \\
$>5.5$ & 13 & $2.6(1.8, \mathrm{NA})$ \\
\hline
\end{tabular}

Figure 1B. Kaplan-Meier Curve of Overall Survival

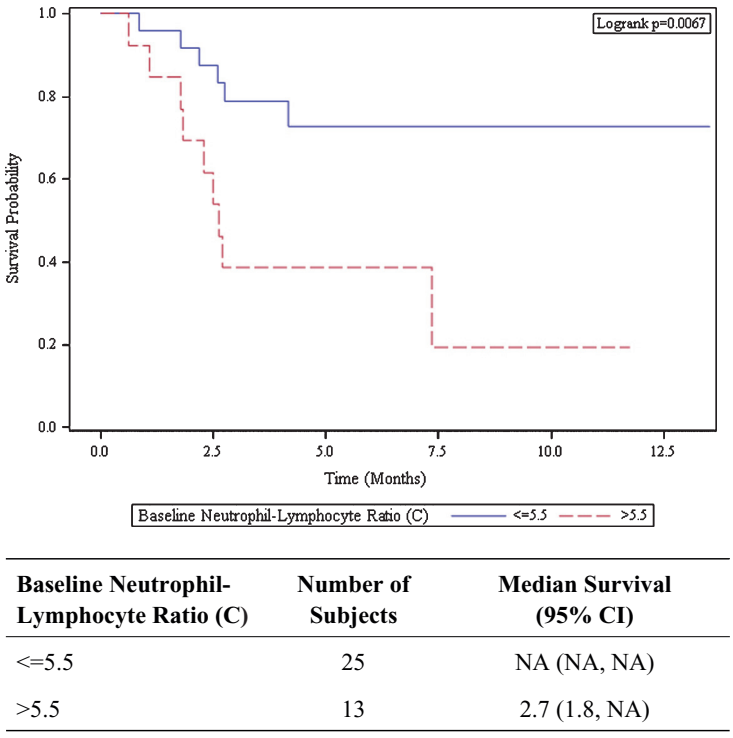

significantly associated with a prolonged OS with a hazard ratio of 0.06 (95\% CI $0.01-0.049 ; \mathrm{p}=0.009)$.

Conclusion: Pre-treatment NLR less than or equal to 5.5 is associated with superior PFS and OS. NLR can be used as a biomarker for prognosis in patients with $\mathrm{mRCC}$ and should be further validated in larger cohorts and in prospective studies.

\section{8}

\section{Association between stool bacteriomic profile and response to sunitinib in metastatic renal cell carcinoma ( $\mathrm{mRCC}$ )}

Dizman, Nazli (City of Hope Comprehensive Cancer Center, Duarte, CA, USA); Poroyko, Valeriy (Department of Medical Oncology and Experimental Therapeutics, City of Hope Comprehensive Cancer Center, Duarte, CA, USA); Wong, Hae Jung (Beckman Research Institute, City of Hope Comprehensive Cancer Center, Duarte, CA, USA); Decat Bergerot, Cristiane (Department of Medical Oncology and Experimental Therapeutics, City of Hope Comprehensive Cancer Center, Duarte, CA, USA); Gustavo Bergerot, Paulo; Maia Caitano, Manuel (Department of Medical Oncology and Experimental Therapeutics, City of Hope Comprehensive Cancer Center, Duarte, CA, USA); Hsu, JoAnn (Department of Medical Oncology and Experimental Therapeutics, City of Hope Comprehensive Cancer Center, Duarte, CA, USA); Frankel, Paul (Division of Biostatistics, Department of Information Sciences, City of Hope Comprehensive Cancer Center, Duarte, CA, USA); Jones, Jeremy (Beckman Research Institute, City of Hope 
Comprehensive Cancer Center, Duarte, CA, USA); Salgia, Ravi (Department of Medical Oncology and Experimental Therapeutics, City of Hope Comprehensive Cancer Center, Duarte, CA, USA); Pal, Sumanta Kumar (Department of Medical Oncology and Experimental Therapeutics, City of Hope Comprehensive Cancer Center, Duarte, CA, USA)

Background: Emerging clinical evidence suggests a link between stool microbiome composition and immunotherapy response (Wargo et al ASCO-SITC 2017). In the context of mRCC, where vascular endothelial growth factor (VEGF)-targeted therapies represent a cornerstone of treatment, it is unclear how the microbiome may influence clinical outcome.

Methods: Patients (pts) with mRCC being treated with sunitinib were included. Five consecutive stool samples were collected at baseline and at weeks 2, 3, 4 and 12 of therapy. Gut microbiota composition were assessed in responders (R: complete/partial response and stable disease) and non-responders (P: primary progression). To assess microbiota composition; microbial DNA was extracted, 16s RNA gene tags (v4) were generated by PCR amplification and sequenced using MiSeq (Illumina). Sequence reads were processed by Mothur software, as described in MiSeq SOP, assembled in Operational Taxonomic Units (OUT), taxonomically annotated to the level of genus and used to construct Bray-Curtis dissimilarity matrix. The similarity of samples was visualized by principle coordinate (PCo) analysis and further confirmed by $\mathrm{k}$-means clustering $(\mathrm{k}=2)$ and ANOSIM tests. Differentially abundant taxa were determined by METASTATS.

Results: Of 6 pts, 4 were evaluable for response. Stool bacteriomic profiling shows that 25,304 OTUs were attributed to 165 genera from 8 phyla. PCo analysis reveals that first two PCo's can explain $51.5 \%$ of data set variation. Subsequent k-means clustering confirms the difference of microbiota in $\mathrm{R}$ and $\mathrm{P}$ groups. The produced clusters are perfectly aligned with R and P groups. ANOSIM test further confirms the significance of this separation $(\mathrm{p}=0.005)$ (Figure 1). The analysis of microbiota composition in $\mathrm{P}$ and $\mathrm{R}$ groups revealed 14 differentially abundant taxonomic units at the genus level, with 5 present at more than $1 \%$ abundance. Namely, Bacteroides, Barnesiellavere and Phascolarctobacterium spp were elevated in group $\mathrm{R}$, while Bifidobacterium spp and Dorea spp were elevated in group $\mathrm{P}(\mathrm{p}<0.01$ for all $)$.
Conclusion: We report the first in-human study suggesting a link between microbiota and response to sunitinib. Although limited by sample size, we identify a significant discrepancy in stool bacteriomic distribution between $\mathrm{P}$ and $\mathrm{R}$.

09

Axitinib and Cabozantinib in the
treatment of sunitinib-refractory
patients with metastatic renal cell
carcinoma (mRCC): Results of
matching adjusted indirect treatment
comparison (MAIC) analysis of AXIS
and METEOR trials

Proskorovsky, Irina (Evidera, St-Laurent, Quebec, Canada); Benedict, Agnes (Evidera, Budapest, Hungary); Negrier, Sylvie (Lyon University, Lyon Cedex 07, France); Cappelleri, Joseph C. (Pfizer Inc, New York, NM, United States); Bargo, Danielle; Desai, Jigar (Pfizer Inc, New York, NY, United States); Larkin, James (The Royal Marsden, London, EN, United Kingdom)

Background: Axitinib and Cabozantinib are approved $2^{\text {nd }}$-line targeted agents frequently used to treat metastatic renal cell carcinoma (mRCC); however, there are no head-to-head trials that compare the relative efficacy of these agents. As baseline characteristics are different between AXIS and METEOR, most notably a significantly higher share of poor risk patients are in AXIS, naïve comparisons are not suitable. The objective of this study was to compare outcomes in sunitinib-refractory (su-r) $\mathrm{mRCC}$ patients treated with axitinib or cabozantinib using a methodology to conduct indirect treatment comparison.

Methods: A matching adjusted indirect comparison (MAIC), which adjusts for imbalances in baseline characteristics between trials, was conducted to compare progression-free survival (PFS) and overall survival (OS) in sunitinib-refractory patients. Individual patient-level data from the sunitinib-refractory axitinib arm of the AXIS trial were weighted to match published patient characteristics of the cabozantinib arm from the METEOR trial to conduct an indirect comparison. Since Karnofsky performance score (KPS) was not collected in AXIS, a conversion from Eastern Cooperative Oncology Group (ECOG) performance status was done to derive Memorial Sloan Kettering Cancer Center (MSKCC) score in 
order to compare patient prognosis between AXIS and METEOR. To assess sensitivity of these results, an alternative mapping was also performed to derive MSKCC score and sensitivity analyses conducted.

Results: After matching, baseline characteristics were balanced between axitinib and cabozantinib patients. No statistical difference was found in the estimated median (m) PFS (mPFS $=7.8$ and 9.1 months) and $\operatorname{mOS}(\mathrm{mOS}=23.8$ and 21.4 months) between axitinib and cabozantinib, respectively. In sensitivity analysis, fewer AXIS patients fell into the MSKCC poor risk category and the estimated treatment effect for both PFS and OS trended towards favoring cabozantinib; however, these results were also not statistically significant.

Conclusions: This analysis suggests no evidence of a statistically significant difference in PFS and OS between axitinib and cabozantinib in sunitinibrefractory $\mathrm{mRCC}$ patients after adjustment for differences in baseline characteristics. OS analyses could not account for likely imbalance in subsequent treatments.

\section{0}

\section{Characterization of Twitter-based dialogue related to renal cell carcinoma (RCC)}

Salgia, Meghan (City of Hope Comprehensive Cancer Center, Duarte, CA, United States); Ashing-Giwa, Kemi; Cotta, Brendan (City of Hope Comprehensive Cancer Center, Duarte, CA, United States); Bergerot, Cristiane Decat (City of Hope Comprehensive Cancer Center, Monrovia, CA, United States); Bergerot, Paulo Gustavo; Dizman, Nazli; Sedrak, Mina S.; Pal, Sumanta K (City of Hope Comprehensive Cancer Center, Duarte, CA, United States)
Background: Social media plays an increasing role in health-related communications, both amongst patients and physicians. We have previously characterized dialogues related to lung cancer on Twitter (Sedrak et al JAMA Oncol 2016), identifying multiple categories of distinct content. We aimed to reproduce these results in the context of RCC.

Methods: Qualitative content analysis of publicly available tweets from August 1 - 22, 2017 containing the word "kidney cancer" was performed. Individual posts were characterized by content domain, and user type, and reviewed by two independent reviewers. Discrepancies were adjusted by consensus. Content was imported from a publicly available Twitter search engine to NVivo 10 for qualitative data analysis.

Results: A total of 2,532 tweets were collected during the study period; 435 were categorized as not related to kidney cancer. As noted in Table 1, the most prevalent content domains related to kidney cancer were support $(29.3 \%)$, treatment $(26.5 \%)$, and general information (13.4\%). Tweets were mostly authored by individuals (41.4\%) and organizations $(41.2 \%)$. Individuals more frequently authored tweets related to support (88.9\%), and organizations those related to treatment $(86.3 \%)$ and general information $(45.4 \%)$.

Conclusion: Twitter was used to receive and give psychosocial support, share personal narratives of cancer, promote prevention, share research findings, and discuss treatment options. The high prevalence of tweets about support was expected. Although, interestingly we found a high frequency of tweets about treatment and clinical trials. These findings suggest that this is a promising area to address health disparities and specific topics, such as goals of care and prognosis, treatment selection, end-of-life care and potential side effects.

Table 1. Content domains of kidney cancer-related tweets.

\begin{tabular}{|l|c|c|c|c|}
\hline \multicolumn{1}{|c}{ Content Domain } & \multicolumn{3}{c}{$\begin{array}{c}\text { User Type } \\
\text { Organization }\end{array}$} & $\begin{array}{c}\text { Media } \\
\text { Frequency, No } \\
(\%)\end{array}$ \\
\hline Support & 547 & 22 & 46 & $615(29.3)$ \\
\hline Prevention & 23 & 1 & 0 & $24(1.1)$ \\
\hline Clinical trials & 59 & 81 & 118 & $258(12.3)$ \\
\hline Treatment & 34 & 480 & 42 & $556(26.5)$ \\
\hline Diagnosis & 94 & 84 & 74 & $252(12.0)$ \\
\hline General information & 85 & 128 & 69 & $282(13.4)$ \\
\hline Symptoms & 1 & 1 & 0 & $2(0.1)$ \\
\hline Donation & 25 & 68 & 15 & $108(5.2)$ \\
\hline
\end{tabular}


11

\section{Checkpoint Inhibitors in the Management of Renal Cell Carcinoma with Sarcomatoid Features}

Pandey, Manu (University of Buffalo, Buffalo, NY, United States); Hanif, Ahmad; Mehta, Rutika; Khan, Sumera; Azabdaftari, Gissou; George, Saby (Roswell Park Cancer Institute, Buffalo, NY, United States)

Background: Metastatic renal cell carcinoma (RCC) with sarcomatoid features carries a poor prognosis and does not respond well to therapy with VEGF inhibitors. Based on results of CheckMate 025 trial, Nivolumab, a programmed cell death-1 (PD-1) inhibitor, was approved for treatment of metastatic RCC in November 2015. However, the efficacy of checkpoint inhibitors in metastatic RCC with sarcomatoid features is unknown.

Methods: We conducted a retrospective chart review of all patients who were diagnosed with metastatic RCC containing sarcomatoid component at Roswell Park Cancer Institute between Jan 2010 to March 2017. Patient characteristics, previous therapies, treatment duration, drug-related adverse events and outcomes including response and overall survival were analyzed.

Results: We identified 34 patients with metastatic RCC who had sarcomatoid component on kidney biopsy. Baseline characteristics of all patients are summarized in Table 1 . Ten patients received Checkpoint Inhibitors (CPI) after failure of first-line therapy while 24 patients were treated without the use of CPI. Median age in CPI group was 61.5 (Range: 42-86) years and 58 (Range: 23-80) years in the non-CPI group. Most common reason for discontinuation of CPI was disease progression. One patient developed grade IV toxicity with colitis, nephritis and pneumonitis that was successfully treated with steroids.

After a median follow up of 10 months, 5 out of 10 patients $(50 \%)$ in the CPI group and 5 out of 24 patients $(20.8 \%)$ in control group are alive. The median survival from the time of diagnosis of metastatic disease was significantly higher in patients who received immunotherapy ( 54 vs. 6 months, $\mathrm{P}<0.001$ ).

Conclusions: Our data indicates that use of checkpoint inhibitors for the treatment of metastatic RCC with sarcomatoid features is associated with improved survival and is relatively well tolerated.

12

\section{Clinical Activity of Nivolumab in Patients with Non-Clear Cell Renal Cell Carcinoma}

Vadim S. Koshkin ${ }^{1}$, Pedro C. Barata ${ }^{1}$, Tian Zhang ${ }^{2}$, Daniel J. George ${ }^{2}$, Michael B. Atkins ${ }^{3}$, William J. Kelly², Nicholas J. Vogelzang ${ }^{4}$, Sumanta K. Pal ${ }^{5}$, JoAnn $\mathrm{Hsu}^{5}$, Leonard J. Appleman', Moshe C. Ornstein', Timothy Gilligan $^{1}$, Petros Grivas ${ }^{1}$, Jorge A. Garcia ${ }^{1}$, Brian I. Rini ${ }^{1}$

${ }^{I}$ Cleveland Clinic Taussig Cancer Institute, Cleveland, $\mathrm{OH}^{2}$ Duke University Medical Center, Durham, NC ${ }^{3}$ Georgetown Lombardi Comprehensive Cancer Center, Washington, $D C^{4}$ Comprehensive Cancer Centers of Nevada, Las Vegas, $N V^{5}$ City of Hope Comprehensive Cancer Center, Duarte, $C A^{6}$ University of Pittsburgh, Pittsburgh, PA

Background: Nivolumab is approved for patients with metastatic renal cell carcinoma (mRCC) refractory to prior antiangiogenic therapy. The clinical activity of nivolumab in patients with non-clear cell RCC subtypes remains unknown as these patients were excluded from the original nivolumab trials.

Table 1 - Baseline Demographics and Clinical Characteristics

\begin{tabular}{|c|c|c|c|}
\hline Characteristic & $\begin{array}{l}\text { Patients who received CPI } \\
\qquad(\mathrm{N}=10)\end{array}$ & $\begin{array}{l}\text { Patients who did not receive CPI } \\
\qquad(\mathrm{N}=24)\end{array}$ & $\begin{array}{c}\text { Total } \\
(\mathrm{N}=34)\end{array}$ \\
\hline Median age in years (range) & $61.5(42-86)$ & $58(23-80)$ & $59(23-86)$ \\
\hline \multicolumn{4}{|l|}{ Sex - no. $(\%)$} \\
\hline $\begin{array}{l}\text { Male } \\
\text { Female }\end{array}$ & $\begin{array}{l}7(70 \%) \\
3(30 \%)\end{array}$ & $\begin{array}{l}12(50 \%) \\
12(50 \%)\end{array}$ & $\begin{array}{l}19(55.88 \%) \\
15(44.22 \%)\end{array}$ \\
\hline \multicolumn{4}{|l|}{ Race - no. (\%) } \\
\hline $\begin{array}{l}\text { White } \\
\text { Other }\end{array}$ & $\begin{array}{l}9(90 \%) \\
1(10 \%)\end{array}$ & $\begin{aligned} 23 & (96 \%) \\
1 & (4 \%)\end{aligned}$ & $\begin{array}{c}32(94.12 \%) \\
2(5.88 \%)\end{array}$ \\
\hline
\end{tabular}


Methods: Patients from 6 centers in the United States (Cleveland Clinic, Duke, Georgetown, Comprehensive Cancer Centers of Nevada, City of Hope and University of Pittsburgh) who received at least one dose of nivolumab for non-clear cell $\mathrm{mRCC}$ between 12/2015 and 06/2017 were identified. A retrospective analysis including patient characteristics, objective response rate according to RECIST v1.1 and treatment-related adverse events (TRAEs) was undertaken. To be considered eligible for response assessment, patients needed to have at least one scan following initiation of nivolumab treatment or to have had clinical progression following initiation of nivolumab as assessed by the treating physician.

Results: Forty-one patients were identified. Median age was 58 years (33-82), 71\% were male, and majority had ECOG PS 0 (40\%) or 1 (47\%). Patient population was $67 \%$ Caucasian, 25\% African American and 8\% Hispanic. Histology included 16 papillary, 14 unclassified, 5 chromophobe, 4 collecting duct, 1 Xp11 translocation and 1 MTSCC (mucinous tubular and spindle cell carcinoma). Most patients had prior nephrectomy $(73 \%)$ and had received 1 $(62 \%)$ or $2(20 \%)$ prior systemic therapies, most commonly sunitinib (63\%), pazopanib $(27 \%)$ or axitinib (10\%). Among 35 patients evaluable for best response, 7 (20\%) had PR and $10(29 \%)$ had SD. No CRs were observed. Responses were observed in unclassified (4), papillary (2) and collecting duct subtypes (1). Among 4 evaluable patients with chromophobe histology, 3 patients had SD although no objective responses were observed. In the entire cohort, median follow-up was 8.5 months and median treatment duration was 3.0 months. Median PFS was 3.5 months and median OS was not reached. Among responders, median time to best response was 5.1 months, and median duration of response was not reached as only 2 of 7 responders had disease progression during follow-up. Four patients were continued on nivolumab treatment beyond radiographic progression. The majority of patients who had disease progression on nivolumab received subsequent systemic treatment (18 of 27 patients). TRAEs of any grade were noted in $37 \%$ of patients and most commonly included fatigue $(12 \%)$, pyrexia $(10 \%)$, rash $(10 \%)$ and hypothyroidism (7\%). Nivolumab treatments were postponed in $34 \%$ and discontinued in $15 \%$ of patients due to intolerance. No treatment-related deaths were observed.

Conclusions: Nivolumab monotherapy demonstrated objective responses and was well tolerated in a heterogeneous population of patients with non-clear cell $\mathrm{mRCC}$. In the absence of available prospective data, this study lends support to the use of nivolum$\mathrm{ab}$ in treatment-refractory patients with metastatic non-clear cell RCC.

\section{3}

\section{Clinical outcome of patients with metastatic Renal Cell Carcinoma (mRCC) progressing on front-line combination regimens that include checkpoint inhibitors}

Barata, Pedro (Cleveland Clinic Taussig Cancer Institute, Cleveland, OH, United States); Gomez de Liano, Alfonso (Barts Cancer Institute, London, EN, United Kingdom); Mendiratta, Prateek (Taussig Cancer Institute, Cleveland Clinic, Cleveland, $\mathrm{OH}$, United States); Szabados, Bernadett; Crolley, Valerie (Barts Cancer Institute, London, EN, United Kingdom); Wood, Laura (Taussig Cancer Institute, Cleveland Clinic, Cleveland, OH, United States); Zanick, Beth (Cleveland Clinic, Cleveland, OH, United States); Allman, Kim; Tyler, Alison (Taussig Cancer Institute, Cleveland Clinic, Cleveland, OH, United States); Martin, Allison (Taussig Cancer Institute, Cleveland Clinic, Cleveland, United

States); Gilligan, Timothy; Grivas, Petros; Ornstein, Moshe; Garcia, Jorge (Taussig Cancer Institute, Cleveland Clinic, Cleveland, OH, United States); Powles, Thomas (Barts Cancer Institute, London, EN, United Kingdom); Rini, Brian (Taussig Cancer Institute, Cleveland Clinic, Cleveland, OH, United States)

Introduction: There are multiple clinical trials in mRCC investigating different combination (COMBO) regimens that include checkpoint inhibitor(s). The clinical outcome of patients on systemic therapy after these $\mathrm{COMBO}$ regimens remains undetermined.

Methods: Patients with advanced, clear-cell mRCC enrolled in one of seven clinical trials investigating a COMBO regimen at two different institutions (Cleveland Clinic Taussig Cancer Institute, Barts Cancer Institute) were retrospectively identified. Baseline characteristics and clinical outcome of subsequent therapy including best objective response according to RECIST v1.1, progression-free survival (PFS) and adverse events using CTCAE v4.0 were collected.

Results: From a total of 89 patients enrolled, 34 patients had RECIST-defined progressive disease (PD) on COMBO. Six patients were excluded from this 
analysis (5 patients remained on all/part of the COMBO regimen after coming off trial and 1 patient died before starting subsequent therapy). Twentyeight patients, median age 58 (41-77), 86\% male, $71 \%$ ECOG 0, 54\% IMDC intermediate risk, were thus identified who were treated with at least one line of subsequent systemic therapy.

Prior COMBO regimens included atezolizumab/ bevacizumab $(\mathrm{n}=17)$, nivolumab/ipilimumab $(\mathrm{n}=$ 10) and axitinib/avelumab $(n=1)$. Approximately two-thirds of patients $(68 \%)$ had prior nephrectomy, and the most common sites of distant metastases included lung (79\%), lymph node $(57 \%)$ and bone (36\%). All except one patient received COMBO in the front-line setting.

All patients received one subsequent therapy (axitinib $n=15$; pazopanib $n=7$; sunitinib $n=3$; cabozantinib $n=3$ ) after progression on COMBO, eleven patients were treated with a second subsequent therapy and five patients were treated with 3 or more subsequent lines of treatment. For patients with available response $(\mathrm{n}=23)$, the overall best response for the first subsequent therapy was PR (22\%), SD (52\%) and PD (13\%). Median PFS for the first subsequent therapy after COMBO was 6.4 months (CI $95 \%$ 4.6-8.2) with 6 patients remaining on treatment. The median PFS for patients previously treated with a combination of immune-VEGF was 5.6 months (CI 95\% 3.2-8.0) and 7.6 months (CI 95\% 4.2-11.0) for patients treated with prior combination immunotherapy $(\mathrm{p}=0.303)$. The most frequent treatment-related adverse events (G3/4) observed with first subsequent therapy were diarrhea $(7 \%)$ and LFT elevation (7\%). Two patients discontinued treatment due to toxicity.

Conclusions: VEGF-TKIs have clinical activity in $\mathrm{mRCC}$ refractory to COMBO therapy, possibly impacted by the mechanism of prior COMBO therapy. Subsequent therapy was in general well-tolerated.

\section{4}

\section{Clinical outcomes of patients treated with local therapies with oligometastatic renal cell carcinoma (mRCC).}

\author{
Mendiratta, Prateek ${ }^{1}$, Gregory Videtic ${ }^{1}$, Timothy \\ Gilligan $^{1}$, Moshe C. Ornstein ${ }^{1}$, Petros Grivas ${ }^{1}$, \\ Jorge Garcia ${ }^{1}$ Brian I. Rini ${ }^{1}$
}

Affiliations: ${ }^{1}$ Cleveland Clinic Taussig Cancer Institute, Cleveland, OH, USA

Background: In a subset of patients with oligometastatic (mRCC), there may be a role for local therapy in an attempt to delay the need for systemic therapy. Techniques like stereotactic body radiotherapy (SBRT) have shown promise in achieving local control in RCC. We review our institutional experience of the use and outcomes for patients with mRCC treated with local therapies.

Material/Methods: An IRB-approved retrospective analysis of the electronic medical record (including imaging) of mRCC patients treated at the Cleveland Clinic was carried out to identify those who received local therapies (such as SBRT, cryoablation, radiofrequency ablation (RFA), intensity-modulated radiation therapy (IMRT), and microwave ablation) in the treatment of non-CNS, non-bone oligometastatic disease. Variables analyzed included baseline patient, tumor and treatment characteristics, outcomes, and toxicities graded per CTCAEv4. Patients receiving local therapies to CNS lesions or bone or for palliation of symptoms were excluded.

Results: From 2008-2017, a total of 14 patients met criteria for analysis. Median age was 64 years (range 50-76), 78.6\% were men, all had clear cell RCC. Median follow-up from diagnosis of metastatic disease to last follow up was 39.5 months (range 1-136).

A total of 19 lesions were treated (84\% lung, 11\% liver, and 5\% renal bed). Treatments were SBRT (74\%), cryoablation (11\%), IMRT (5\%), RFA (5\%), and microwave ablation (5\%). Three patients (16\%) had received one prior systemic therapy (sunitinib, IL-2, and sorafenib) and were treated with local therapy due to oligoprogression. Two patients received prior neo-adjuvant and adjuvant systemic therapy on clinical trials. One patient had prior metastasectomy. SBRT dose schedules ranges 30Gy in 1 fraction to 60 Gy in 3 fractions.

Treatment adverse events were limited (26\% G13 ) including one patient with grade 3 pleural effusion post cyroablation, one patient with grade 2 pneumonitis post SBRT, and 3 patients with grade 1 fatigue post SBRT (all after treatment to lung lesions).

The median time from local therapy to systemic or local progression was 10 months (range 3-60). Seven patients $(50 \%)$ progressed systemically at a median of 9 months after local therapy and one patient had 
local progression (at 60 months) in the liver retreated successfully with microwave ablation. Nine patients $(64 \%)$ have not required further systemic therapy. The median number of further systemic therapies used upon progression was one. Three patients died due to complications of their disease.

Conclusions: Local therapies are safe and feasible for visceral oligometastatic disease with the majority of patients demonstrating local control with minimal toxicity. Prospective studies are warranted to determine if local therapy in mRCC alters the natural history and/or can delay the need for systemic therapy.

\section{5}

\section{Clinicopathological characterization and oncologic outcomes of metastatic small renal masses}

Renzo G. DiNatale ${ }^{1}$, Alejandro Sanchez ${ }^{1}$, Kyle A. Blum ${ }^{1}$, Nirmal T John' ${ }^{1}$, Maria Becerra ${ }^{1}$, Wanling Xie ${ }^{2}$, Toni K. Choueiri², Daniel Heng', Paul Russo ${ }^{1}$, A. Ari Hakimi ${ }^{1}$

${ }^{I}$ Memorial Sloan Kettering Cancer Center, New York, NY, USA

${ }^{2}$ Dana-Farber Cancer Institute, Boston, MA, USA.

${ }^{3}$ Tom Baker Cancer Center, Calgary, Alberta, Canada.
Funding: Ruth L. Kirschstein Research Service Award T32CA082088 (A.S.)

Introduction: Due to the increased use of imaging techniques, the incidence of small renal masses (SRMs, $\leq 4 \mathrm{~cm}$ ) has been steadily rising. Most of these SRMs represent an early-stage renal cell carcinoma (RCC) and have excellent oncologic outcomes following partial nephrectomy. However, around 2\% of patients with SRMs present with metastatic disease, leading to poor survival outcomes. We aim to describe the clinicopathological characteristics of patients with metastatic SRMs (mtSRMs) and compare their oncologic outcomes to those with larger metastatic RCCs.

Method: We retrospectively reviewed the medical records of 685 patients with RCC who underwent partial or radical nephrectomy between Jan-1998 and Jan-2016 and developed metastatic disease. Patients with tumors $\leq 4 \mathrm{~cm}$ (T1a) with either synchronous or metachronous metastases were considered mtSRMs. Patients were categorized as having either synchronous or metachronous metastases based on a 3-month cut-off point from the date of presentation to the date of metastasis. Patients with metachronous metastases ( $>3$ months after surgery) were then grouped according to their time to metastasis in either early $(<2$ years) or late $(>2$ years $)$ metastasis.

Table 1: Comparison of baseline characteristics among patients with metastatic RCC by size.

\begin{tabular}{|c|c|c|c|c|}
\hline & $\begin{array}{l}\leq 4 \mathrm{~cm} \\
(\mathrm{n}=93)\end{array}$ & $\begin{array}{l}>4 \mathrm{~cm} \\
(\mathrm{n}=592)\end{array}$ & $\begin{array}{l}\text { Difference }(95 \% \mathrm{Cl}) \\
{[\leq 4->4]}\end{array}$ & $\mathrm{p}$-value \\
\hline Mean age at time of surgery $(y)$ & 63.79 & 61.01 & $-2.78(-5.22,-0.32)$ & 0.02 \\
\hline$\%$ Males & 63.4 & 74.5 & $-11.05(-22.07,-0.03)$ & 0.03 \\
\hline Mean BMI (kg/m2) & 28.78 & 28.80 & $-0.02(-1.22,1.25)$ & 0.97 \\
\hline Mean size $(\mathrm{cm})$ & 2.97 & 9.06 & $-6.09(-5.77,-6.40)$ & $<0.001$ \\
\hline$\%$ Clear cell histology & 88.17 & 91.39 & $-3.21(-10.78,4.35)$ & 0.41 \\
\hline \% Partial nephrectomies & 58.06 & 8.45 & $49.62(38.72,60.52)$ & $<0.001$ \\
\hline$\%$ pT3 tumors & 30.11 & 67.91 & $-37.80(-27.12,-48.47)$ & $<0.001$ \\
\hline$\%$ Sarcomatoid features & 3.23 & 15.37 & $-12.15(-6.90,-17.39)$ & 0.002 \\
\hline$\%$ High grade $(3-4)$ & 34.41 & 66.39 & $-31.98(-20.98,-42.98)$ & $<0.001$ \\
\hline$\%$ Metachronous metastasis & 52.69 & 44.26 & $8.43(-3.09,19.96)$ & 0.16 \\
\hline Mean time to metastasis $(n=311)$ & 49.48 & 38.05 & $11.43(-0.68,23.54)$ & 0.06 \\
\hline Median overall survival (months) & 88.7 & 43.6 & HR $0.55(0.41-0.73)$ & $<0.001$ \\
\hline
\end{tabular}


We compared baseline characteristics of these patients with those who had larger tumors $(\leq 4 \mathrm{~cm}$ vs $>4 \mathrm{~cm}$ ). Statistical analysis of baseline values was done using chi-squared and t-tests. Survival analysis was done using log-rank tests and constructing Kaplan-Meier curves for the different groups.

Results: From our initial cohort of metastatic patients with RCC ( $\mathrm{n}=685)$, we identified $93 \mathrm{mtSRMs}$ $(13.58 \%)$. We then proceeded to compare baseline characteristics between mtSRMs and larger tumors (Table 1). The mtSRM cohort had younger patients $(-2.78 \mathrm{y}, \mathrm{p}=0.02)$ and fewer men $(-11.05 \%, \mathrm{p}=0.03)$. The proportion of patients with pT3 tumors $(-37.8 \%$, $\mathrm{p}<0.001)$, sarcomatoid features $(-12.15 \%, \mathrm{p}=0.002)$ and high grade $(-31.9 \%,<0.001)$ was significantly lower in the mtSRM group. The time to metastasis was not significantly different between both groups $(p=0.06)$. However, survival analysis showed that the hazard ratio of death in the SRM group was $45 \%$ less when compared to bigger tumors $(\mathrm{p}<0.001)$.

Conclusion: Clinicopathological differences between patients with mtSRMs and those with larger RCCs could not fully explain the differences in oncologic outcomes between these two groups. Studies with larger clinical cohorts including the use of molecular and genomic biomarkers are required to better characterize these patients and understand the aggressive behavior of mtSRMs.

\section{6}

\section{Comparative Genomic Profiling of Matched Primary and Metastatic Tumors in Renal Cell Carcinoma}

Maria F. Becerra ${ }^{\mathrm{a}, \mathrm{b}}$, Ed Reznik ${ }^{\mathrm{c}, \mathrm{d}}$, Almedina Redzematovic $^{\mathrm{e}}$, Daniel M. Tennenbauma, Mahyar Kashan ${ }^{\mathrm{a}}$, Mazyar Ghanaat ${ }^{\mathrm{a}}$, Jozefina Casuscellia,f, Brandon Manley, Philip Jonsson ${ }^{\mathrm{d}, \mathrm{g}}$, Renzo G. DiNatale ${ }^{\mathrm{a}}$, Kyle A. Bluma, Jeremy C. Durack ${ }^{\mathrm{h}}$, Stephen B. Solomon $^{\text {h }}$, Maria E. Arcila ${ }^{\text {i }}$ Caitlin Bourque , Nick Soccic, Maria I. Carlo ${ }^{\mathrm{e}}$, Chung-Han Lee ${ }^{\mathrm{e}}$, Martin H. Voss ${ }^{\mathrm{e}}$, Darren R. Feldman', Robert J. Motzere, Jonathan A. Coleman ${ }^{a}$, Paul Russo ${ }^{a}$, Emily H. Cheng',

A. Ari Hakimi ${ }^{\mathrm{a}}$, and James J. Hsieh ${ }^{\mathrm{j}, *}$

a Urology Service, Department of Surgery, Memorial Sloan Kettering Cancer Center, New York, NY, USA ${ }^{b}$ Department of Urology, Miller School of Medicine University of Miami, Miami, FL, USA ${ }^{c}$ Center for Molecular Oncology, Memorial Sloan Kettering Cancer Center, New York, NY, USA
${ }^{d}$ Department of Epidemiology and Biostatistics, Memorial Sloan Kettering Cancer Center, New York, New York, USA

${ }^{e}$ Genitourinary Oncology Service, Department of

Medicine, Memorial Sloan Kettering Cancer Center, New York, NY, USA

${ }^{f}$ Department of Urology, Ludwig-Maximilians University, Munich, Germany

${ }^{g}$ Human Oncology and Pathogenesis Program, Memorial Sloan Kettering Cancer Center, New York, NY, USA

'Interventional Radiology Service, Department of Radiology, Memorial Sloan Kettering Cancer Center, New York, NY, USA

${ }^{i}$ Department of Pathology, Memorial Sloan Kettering Cancer Center, New York, NY, USA

${ }^{j}$ Molecular Oncology, Department of Medicine, Siteman Cancer Center, Washington University, St. Louis, MO, USA

Background: Next-generation sequencing (NGS) studies of matched pairs of primary and metastatic tumors in renal cell carcinoma (RCC) have been limited to small cohorts.

Objective: To evaluate the discordance of somatic mutations between matched primary and metastatic RCC tumors.

Materials \& Methods: Primary tumor (P), metastasis (M), and germline DNA from 60 patients with RCC was subject to NGS with a targeted exon capture-based assay of 341 cancer-associated genes. Somatic mutations were called using a validated pipeline.

Statistical analysis: Mutations were classified as shared $(S)$ or private $(P r)$ in relation to each other within individual P-M pairs. Concordance score was calculated as $(\mathrm{S}-\mathrm{Pr}) /(\mathrm{S}+\mathrm{Pr})$. To calculate enrichment of private/shared mutations for a particular gene, we calculated a two-sided p-value from a binomial model for each gene with at least 10 somatic mutation events, and implemented a separate permutation test procedure. P-values were adjusted for multiple hypothesis testing using the BenjaminiHochberg procedure. The mutation discordance was calculated using Mann-Whitney $U$ tests according to gene mutations or metastatic sites.

Results: Twenty-one (35\%) pairs showed private mutations in both primary and metastasis. Of the remaining 39 (65\%) pairs, 14 (23\%) had private mutations specific to primary tumors, $12(20 \%)$ had private mutations to metastases, and $13(22 \%)$ had identical somatic mutations. No individual gene mutation was preferentially enriched in either primary 
or metastatic samples. P-M pairs with SETD2 mutations demonstrated higher discordance than pairs with wild-type SETD2. We observed that patients who received therapy prior to sampling of the primary or metastatic tissue had higher concordance of mutations between P-M pairs than patients who did not (Mann-Whitney p-value 0.088 ).

Conclusions: Our data show mutation discordance within matched P-M RCC tumor pairs. As most contemporary precision medicine trials do not differentiate mutations detected in primary or metastatic tumors, the prognostic and predictive value of mutations in primary vs. metastasis warrants further investigation.

\section{7}

\section{DART Study: A phase 2 randomized trial of dalantercept plus axitinib versus placebo plus axitinib in advanced renal cell carcinoma (RCC): Results from the part 2 placebo- controlled trial.}

\author{
Martin H. Voss ${ }^{1}$, Nicholas J. Vogelzang ${ }^{2}$, Mayer \\ Fishman $^{3}$, Robert S. Alter ${ }^{4}$, Brian I. Rini ${ }^{5}$, J. Thaddeus \\ Beck $^{6}$, Monika Joshi ${ }^{7}$, Michael B. Atkins ${ }^{8}$, Xiaosha \\ Zhang ${ }^{9}$, Chad Glasser ${ }^{9}$, Musa Mutyaba ${ }^{9}$, Brian Vidal ${ }^{9}$, \\ Matthew L. Sherman ${ }^{9}$, Rupal S. Bhatt ${ }^{10}$, Elizabeth R. \\ Plimack $^{11}$, DART Study Group \\ ${ }^{1}$ Memorial Sloan Kettering Cancer Center, New York, NY; \\ ${ }^{2}$ Comprehensive Cancer Centers of Nevada, Las Vegans, \\ NV; ${ }^{3}$ Moffit Cancer Center, Tampa, FL; ${ }^{4}$ John Theurer \\ Cancer Center Hackensack UMC, Hackensack, NJ; \\ ${ }^{5}$ Cleveland Clinic Taussig Cancer Institute, Cleveland, \\ OH; ${ }^{6}$ Highlands Oncology Group, Fayetteville, AR; \\ ${ }^{7}$ Penn State Hershey Cancer Institute, Hershey, PA; \\ ${ }^{8}$ Georgetown University Medical Center, Washington, \\ DC; ${ }^{9}$ Acceleron Pharma, Cambridge, MA; ${ }^{10}$ Beth Israel \\ Deaconess Medical Center, Boston, MA; ${ }^{11}$ Fox Chase \\ Cancer Center, Philadelphia, PA
}

Background: Agents targeting the vascular endothelial growth factor (VEGF) pathway in patients (pts) with advanced renal cell carcinoma (RCC) have limited activity due to the development of alternate angiogenic escape pathways, suggesting the need for therapeutic approaches that can augment angiogenic blockade. Activin receptor-like kinase 1 (ALK1) is a type I receptor of the TGF- $\beta$ superfamily and is a novel angiogenesis target involved in blood vessel maturation. Concurrent targeting of ALK1 and vascular endothelial growth factor receptor (VEGFR) signaling results in dual angiogenic blockade and augmented inhibition of tumor growth in RCC xenograft models. Dalantercept is an ALK1 receptor-fusion protein that acts as a ligand trap and achieved additive efficacy with a VEGFR tyrosine kinase inhibitor (TKI) in RCC xenograft models. We conducted a Phase 1 trial testing the combination of dalantercept plus axitinib in pts previously treated with other VEGF-directed agents (Voss et al., 2016), followed by this double-blind placebo controlled randomized phase 2 trial.

Methods: The primary objective of this study was to determine whether treatment with dalantercept plus axitinib prolonged PFS compared to axitinib plus placebo in pts with advanced RCC. Pts were randomized 1:1 to receive either dalantercept $(0.9 \mathrm{mg} /$ $\mathrm{kg}$ was selected based on Part 1) or placebo SC Q3W plus axitinib $5 \mathrm{mg}$ PO BID. Key eligibility: predominantly clear cell RCC, 1 prior VEGFR TKI, $<3$ prior treatments, and ECOG $\leq 1$. Clinicaltrials.gov NCT01727336.

Results: At the time of the primary data analysis, a total of 119 pts were enrolled (58 randomized to dalantercept + axitinib and 61 to placebo + axitinib) and achieved median PFS 6.8 months for dalantercept + axitinib vs 5.6 months for placebo + axitinib. Dalantercept + axitinib did not decrease the rate of disease progression or death (HR 1.11, 2-sided 95\% CI [0.71, 1.73], 1-sided p-value 0.67). The confirmed objective response rate (ORR) was $19 \%$ for dalantercept + axitinib vs $25 \%$ for placebo + axitinib (p-value 0.43 ). In the subgroup of pts who received 2 or more prior systemic anti-cancer therapies, median PFS was 8.1 months for dalantercept + axitinib vs 7.0 months for placebo + axitinib (HR 0.78, 2-sided $95 \%$ CI [0.33, 1.87], 1-sided p-value 0.29).

The number of pts reporting at least 1 grade $3 \mathrm{AE}$ regardless of causality was similar in both study arms (59 vs $64 \%$, dalantercept + axitinib vs placebo + axitinib). The safety profile was similar to that seen in the Phase 1 trial and previous dalantercept trials.

Conclusions: In this double-blind placebo-controlled trial, the addition of dalantercept to standard of care axitinib did not lead to a statistically significant increase in PFS in previously treated pts with RCC. Based on the lack of efficacy, the development of dalantercept has been discontinued. 
18

\section{Deferred Systemic Therapy (DST) for Metastatic Renal Cell Carcinoma: Preliminary Prospective Experience}

Harrison, Michael R. (Duke University Medical Center, Durham, NC, United States); Costello, Brian A. (Mayo Clinic, Rochester, MN, United States); Bhavsar, Nrupen A. (Duke University Medical Center, Durham, NC, United States); Vaishampayan, Ulka (Karmanos Cancer Institute, Detroit, MI, United States); Pal, Sumanta K. (City of Hope, Duarte, CA, United States); Zakharia, Yousef (University of Iowa Hospitals and Clinics, Iowa City, IA, United States); Jim, Heather; Fishman, Mayer N. (Moffitt Cancer Center, Tampa, FL, United States); Molina, Ana M. (Weill Cornell Medicine, New York, NM, United States); Kyriakopoulos, Christos (University of Wisconsin, Madison, WI, United States); Tsao, Che-Kai (Tisch Cancer Institute, New York, NY, United States); Appleman, Leonard J. (UPMC Cancer Pavilion, Pittsburgh, PA, United States); Gartrell, Benjamin A. (Montefiore Hospital and Medical Center, Bronx, NY, United States); Hussain, Arif (University of Maryland, Baltimore, United States); Stadler, Walter M. (The University of Chicago, Chicago, IL, United States); Agarwal, Neeraj (Huntsman Cancer Institute, Salt Lake City, UT, United States); Pachynski, Russell (Washington University School of Medicine, St Louis, MO, United States); Hutson, Thomas E. (Baylor Sammons Cancer Center-Texas Oncology, Dallas, TX, United States); Hammers, Hans J. (UT Southwestern, Dallas, TX, United States); Ryan, Christopher W. (Oregon Health and Science University, Portland, OR, United States); Mardekian, Jack; Singh, Kanwarjit; Borham, Azah (Pfizer Inc, New York, NY, United States); George, Daniel J. (Duke University Medical Center, Durham, NC, United States)

Background: Metastatic renal cell carcinoma (mRCC) is a heterogeneous disease. In a subset of patients with slow-growing metastases, systemic therapy (ST) may be deferred. Some retrospective data and one prospective clinical trial have been reported on deferred systemic therapy (DST) in mRCC. Using the Metastatic Renal Cell Cancer Registry (MaRCC) we report our preliminary data analysis on baseline characteristics and demographics and reasons for the treating physician's management decision to defer therapy as the initial management decision.

Methods: MaRCC Registry enrolled 502 evaluable patients at 46 US academic $(\mathrm{N}=20)$ and community $(\mathrm{N}=26)$ sites from $3 / 24 / 2014$ to $12 / 22 / 2016$ and will include $\geq 3$ years of follow-up. Eligible patients were age $\geq 18$ years with a diagnosis of $\mathrm{mRCC}$ and no prior ST for mRCC at study entry. Patients not on ST but rather undergoing observation were also permitted to enroll on the registry. Key endpoints included treatment characteristics (e.g. agents, sequence, duration, reasons for therapy choice and discontinuation), treatment effectiveness (e.g. ORR, PFS, OS), quality of life, medication adherence, and health resource utilization. DST was defined as anything other than ST (e.g. active surveillance, local therapy, etc.) as the initial management decision. Descriptive statistics were used to quantify patient demographic and clinical characteristics. T-tests were used to test for significance between the reasons for choice of ST versus DST, and baseline patient reported outcomes (PRO).

Results: As of the August 4, 2017 data cut off, mean and median follow up for the entire registry cohort were 9.9 and 8.5 months, respectively. At the time of data cut off, 208/502 (41\%) patients had DST as the initial management decision: 73/208 (35\%) patients had crossed over from DST to ST and 135/208 $(65 \%)$ remained in the DST group. In the DST cohort: median follow up from screening and metastatic diagnosis were 9.5 and 17.6 months, respectively; median age 65 years $(\mathrm{Q} 1-3$ range, 5874); $71 \%$ male; $30 \%$ ACAD; $81 \%$ clear cell histology; and $27 \%$ stage IV at diagnosis. Baseline demographic and clinical characteristics were similar between DST, DST-to-ST, and ST groups, except years between initial RCC diagnosis and enrollment (longer in DST group). The most common primary reasons for DST, as assessed by the treating physician, were active surveillance, disease present (37\%); active surveillance, no evidence of disease following procedure (20\%); and local therapy $(13 \%)$. At baseline, PRO questionnaires demonstrated significantly better quality of life (FACT-G) and kidney cancer symptoms (FKSI-19) in the DST group compared with the $\mathrm{ST}$ group (both $\mathrm{P}<0.05$ ), including among the FACT-G domains of physical, social and functional well-being (all $\mathrm{P}<0.05$ ).

Conclusions: This is the largest prospective experience of DST in mRCC to date. In this preliminary analysis, with median follow up of 8.5 months, $135 / 502(27 \%)$ of treatment-naïve mRCC patients remained on DST. As this data matures over time, future planned analyses in the DST cohort will include outcomes (PFS, OS, time to systemic therapy), longitudinal PROs, prognostic modeling, and biomarker studies. 
19

\section{Do CT perfusion measures differ in primary renal tumors versus metastatic lesions in patients receiving treatment for advanced renal cell carcinoma?}

Fan, Alice (Stanford University, Stanford, CA, United States); Metzner, MS, Thomas (Stanford University, Mountain View, CA, United States); Kino, Aya (Stanford University School of Medicine, Stanford, CA, United States); Sundaram, Vandana (Stanford University, Stanford, CA, United States); Schmiedeskamp, Heiko (Siemens Medical Solutions, Malvern, PA, United States); Desai, Manisha (Stanford University, Palo Alto, CA, United States); Kamaya, Aya (Stanford University, Stanford, CA, United States)

Introduction: Perfusion CT allows for the visualization and quantification of tumor vascularity by measuring blood perfusion in tissues. Due to its highly vascularized nature, renal cell carcinoma (RCC) is especially amenable to visualization with perfusion CT. It has been suggested that measurements of perfusion in metastatic RCC lesions may predict the efficacy of anti-angiogenesis agents. We have previously reported that CT perfusion mea- surements after only 8 days of treatment can correlate with the efficacy of targeted therapy in patients with advanced RCC. We hypothesize that perfusion imaging early during treatment with targeted therapy can detect changes in vascularity in both primary RCC renal lesions and metastatic RCC lesions. We aim to determine if there is a difference in early $\mathrm{CT}$ perfusion measures comparing renal lesions with metastatic lesions during therapy.

Methods: In this IRB-approved prospective study, patients with advanced RCC received a perfusion CT scan prior to treatment (baseline), and 7-10 days after initiating treatment (day 8). Perfusion measurements of tumor vascularity included blood volume (BV), blood flow (BF), mean transit time (MTT), and flow extraction product (FEP). The longest dimension was measured in each lesion. Clinical response was defined based on RECIST 1.1 after 12 weeks of treatment. Univariable logistic regression analysis was used to determine the association of clinical response and tumor location. We evaluated the relationship between tumor location and change in each measure from baseline to day 8. Association between clinical response and each individual measure for each tumor location was evaluated

Figure 1: Distribution of change in measurements by clinical response: metastatic lesions versus kidney lesions

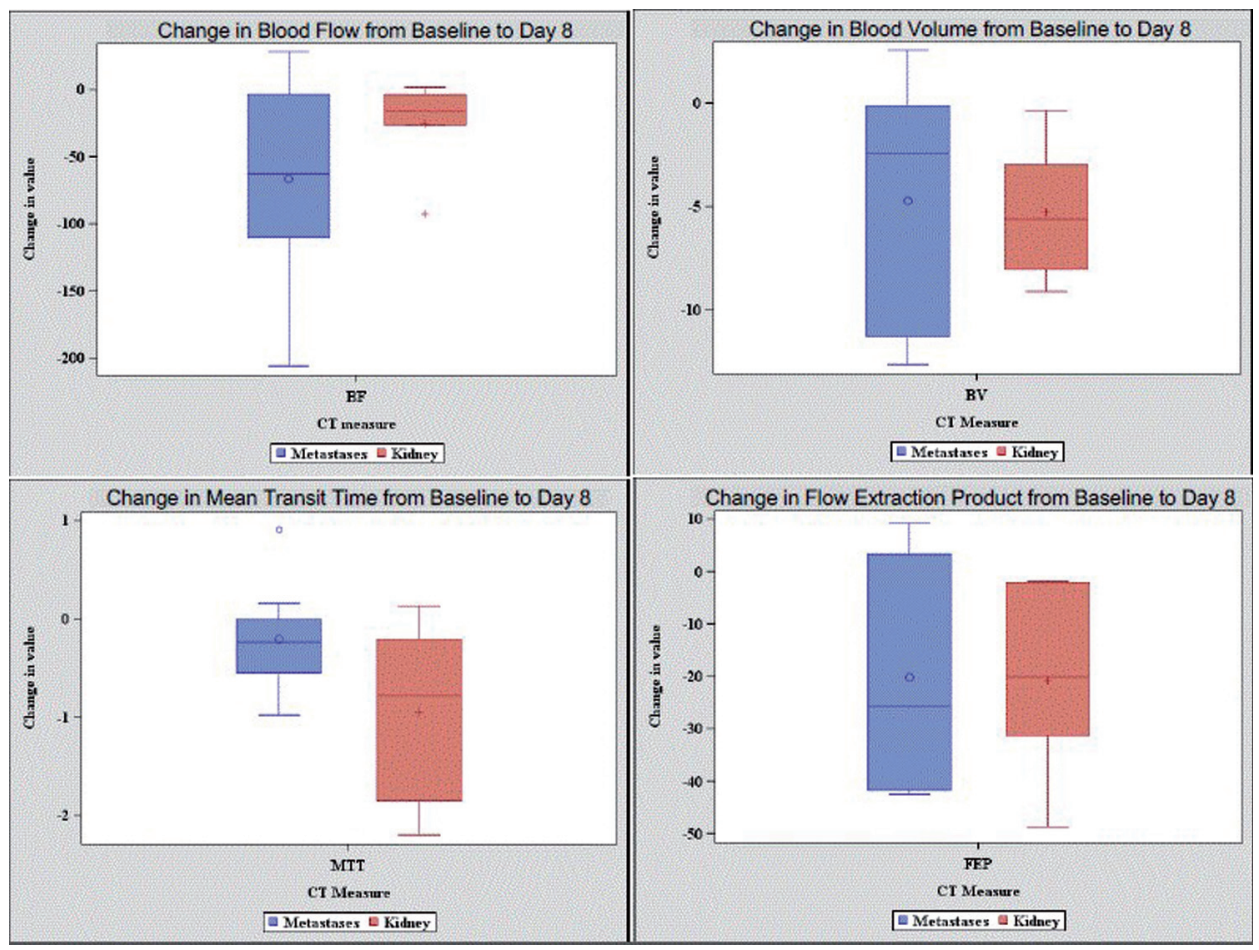


Figure 2: Changes in Blood Volume and Blood Flow by Clinical Response and Tumor Location

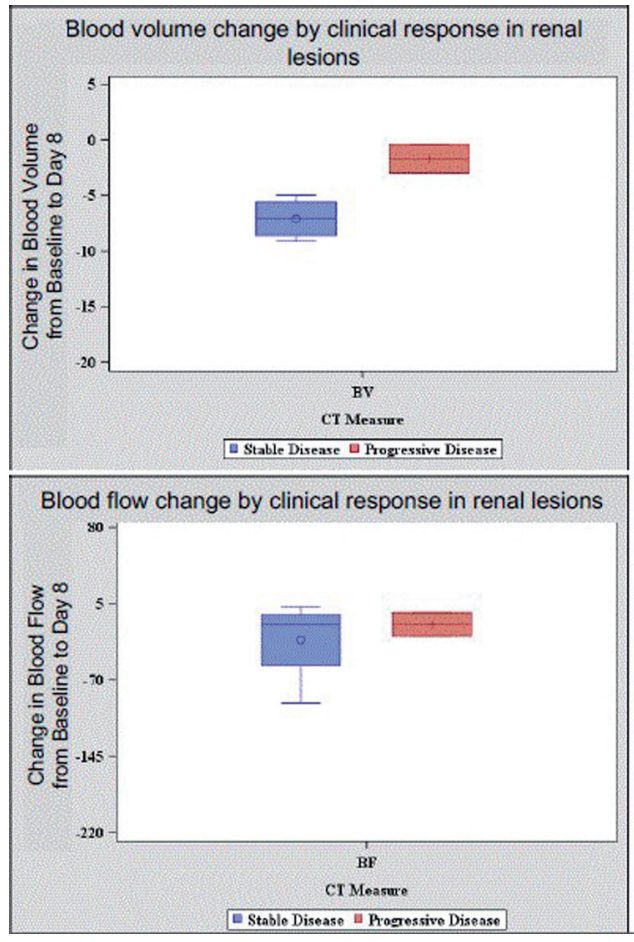

separately (renal lesion or metastatic lesion). Significance testing was assessed at a two-sided alpha level of 0.10 .

Results: 11 patients with advanced RCC who required treatment with anti-angiogenesis agents or immune checkpoint inhibitor were enrolled. 5 patients had primary renal masses imaged with perfusion CT, one patient had both a primary renal mass and a metastatic lesion, and 5 patients had metastatic RCC lesions (in single or multiple sites, including adrenal, pancreas, lung, liver and soft tissue).

At 12 weeks, $67 \%$ of the renal masses had stable RECIST measurements and 33\% had RECIST measurements consistent with progressive disease. Among the metastatic lesions, $25 \%$ had stable measurements and $75 \%$ progressed at 12 weeks. There was no statistically significant association between tumor location (kidney or metastasis) and clinical response (stable or progressive disease) (OR: 6.0 (90\% CI: 0.85-42.5); $\mathrm{p}=0.13$ ).

At the early imaging time point, we were able to quantify changes from baseline to day 8 in tumor vascularity measures, whereas tumor size did not significantly change during this short interval.

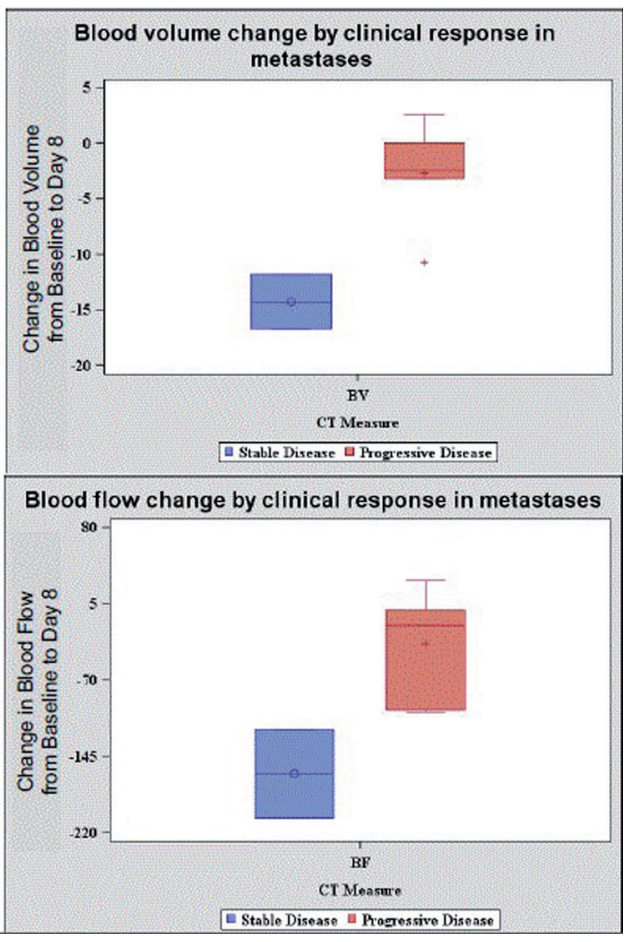

Changes at Day 8 , in BF, BV and FEP measures in metastatic lesions had greater variation compared to renal lesions (Figure 1). Pts with stable disease had greater decreases in $\mathrm{BV}$ and $\mathrm{BF}$ for both renal and metastatic lesions compared to patients with progressive lesions. Further, in patients with stable disease, changes in vascularity were more pronounced in metastatic lesions compared to renal lesions (Figure 2). Our results are consistent with the notion that stabilization of tumor growth by targeted therapy can be associated with decreases in tumor vascularity measurements.

Conclusion: We found that early changes in $\mathrm{BF}$ and $\mathrm{BV}$ in advanced RCC patients were of greater magnitude in patients with stable disease compared to progressive disease. In addition, changes were more pronounced in metastatic tumor sites compared to primary renal tumors. This work suggests that early perfusion changes, especially in metastatic lesions, might be helpful to determine if patients are benefiting from targeted therapy. Further studies are needed to see if CT perfusion measures can be developed as a biomarker to measure early therapeutic response. 
20

\section{Effects of pazopanib (PAZ) and sunitinib (SUN) dose modification on safety and efficacy in patients with metastatic renal cell carcinoma (mRCC) from COMPARZ}

Bjarnason, Georg A (Sunnybrook Odette Cancer Centre, University of Toronto, Toronto, Ontario, Canada); Kollmannsberger, Christian (British Columbia Cancer Agency, Vancouver, BC, Canada); Ahmad, Qasim I. (Novartis Pharmaceuticals Corporation, East Hanover, NJ, United States); Dezzani, Luca; Elmeliegy, Mohamed; Han, Jackie (Novartis Pharmaceuticals Corporation, East Hanover, NJ, United States); Nathan, Paul (Mount Vernon Cancer Centre, Northwood, EN, United Kingdom)

Background: COMPARZ was a randomized, controlled, open label, phase 3 trial that demonstrated comparable efficacy of first line PAZ and SUN, but favorable safety and quality of life profiles for PAZ in patients (pts) with mRCC (NEJM 2013;369:722). We evaluated the relationship between dosing, safety, and efficacy in PAZ and SUN treated pts who did or did not undergo dose reduction or interruption resulting from adverse events (AEs) and other reasons

Methods: The AEs and median progression free survival (mPFS) of PAZ and SUN were evaluated for pts with no, any, 1 , and $\geq 2$ dose reductions or dose interruptions lasting $\geq 7$ days

Results: Similar percentages of pts in the PAZ and SUN groups had a dose interruption ( $44 \%$ vs $49 \%$, respectively) or reduction $(44 \%$ and $51 \%$, respectively). The incidence of AEs in pts from the PAZ and SUN groups with dose modifications was higher compared to those with no dose modifications. Longer mPFS was observed in pts with dose modification (Table). Pts treated with PAZ or SUN with no dose reductions had mPFS of 7.3 months (mos) and 5.5 mos, respectively, whereas pts with any dose reduction had mPFS of 12.5 mos and 13.8 mos, respectively. Similarly, pts treated with PAZ or SUN with no dose interruptions lasting $\geq 7$ days had mPFS of 8.2 mos and 5.6 mos, respectively, whereas those with any dose interruption lasting $\geq 7$ days had mPFS of 12.6 mos and 13.8 mos, respectively. Pts with 2 or more dose interruptions or reductions had mPFS $>16$ mos with both SUN and PAZ

Conclusions: Consistent with previous data for SUN, the current analyses showed longer mPFS with PAZ and SUN when dose modification is required to manage toxicity, suggesting that pts are not disadvantaged by such dose reductions or interruptions. Pts not requiring dose modification may have suboptimal therapeutic drug exposure. Clinical trial information: NCT00720941

\section{1}

\section{Estimating the Social Value Generated by Immunotherapy for Renal Cell Carcinoma Patients}

Sullivan, Jeffrey (Precision Health Economics, Los Angeles, CA, United States); Sexton Ward, Alison; Peneva, Desi (Precision Health Economics, Los Angeles,

\begin{tabular}{lll}
\hline Dose reduction (s), mPFS, (95\% CI) & PAZ, mos & SUN, mos \\
\hline None & $7.3(5.3-8.3)$ & $5.5(4.3-8.1)$ \\
Any & $12.5(10.9-15.0)$ & $13.8(11.1-16.4)$ \\
$\mathbf{1}$ & $11.1(8.3-13.5)$ & $11.1(10.2-13.8)$ \\
$\mathbf{2}$ & $16.4(11.1-18.6)$ & $16.5(11.5-19.3)$ \\
Dose interruption(s) $\geq \mathbf{7}$ days, mPFS, (95\% CI) & & \\
None & $8.2(5.5-8.3)$ & $5.6(5.4-8.2)$ \\
Any & $12.6(9.9-16.4)$ & $13.8(11.1-16.6)$ \\
$\mathbf{1}$ & $8.3(6.0-11.0)$ & $11.0(8.2-14.0)$ \\
$\geq \mathbf{2}$ & $16.7(13.7-19.4)$ & $16.6(13.6-19.6)$ \\
\hline
\end{tabular}

Reused with permission from the American Society of Clinical Oncology (ASCO). This abstract was accepted and previously presented at the 2017 ASCO Annual meeting. All rights reserved 
United States); Yang, Shuo; Rao, Sumati (Bristol-Myers Squibb, Princeton, United States); Figlin, Robert A (Cedars-Sinai Medical Center, Los Angeles, United States)

Background: Recent development of new immunotherapies offers the potential for long-term survival benefits and extended disease control for certain cancers. While the clinical efficacy of these new treatments has been reviewed in literature, the social value generated by new immunotherapies related to long-term clinical benefits has yet been fully explored. This study, using advanced or metastatic renal cell carcinoma (aRCC) as an example, investigated the potential long-term impact of nivolumab on life expectancy and social value, which is defined as the change in monetized qualityadjusted life-years associated with the treatment in question minus the change in medical expenditures inclusive of the projected total treatment cost.

Methods: The Health Economics Medical Innovation Simulation (THEMIS) was used to project the anticipated changes in aRCC patient survival and quality of life post-diagnosis due to the introduction of new treatment options, including nivolumab and new targeted therapies introduced after 2015, over the next 50 years. THEMIS, which tracks a representative sample of patients aged $51+$ years, was used to first estimate longevity for aRCC patients under a baseline scenario without nivolumab or new targeted therapies where mortality and disease stage were estimated using the Surveillance and Epidemiology End Results (SEER) database. The baseline was then compared versus both a new targeted therapy scenario (based on cabozantinib and lenvatinib + everolimus) and two nivolumab scenarios, where survival and patient response rates were based on the CheckMate 025 study. Both nivolumab scenarios assume the same clinical efficacy but differ in the average treatment duration (1 year or 2 years) required to attain full treatment benefit. Other health state transitions were estimated from nationally representative datasets, and a full year of healthy life was valued at $\$ 150,000$ USD.
Results: In the baseline scenario, aRCC patients lose 17 years of life compared with a nationally representative population, which is comparable to published estimates of 13 years for all patients regardless of stage (Burnet NG et al. Br J Cancer 2005;92:2415). Compared with baseline, the nivolumab scenarios extend life expectancy by 1.9 years and provide positive social value over the next 50 years ranging from $\$ 18,783$ to $\$ 33,108$ per aRCC patient and from around $\$ 188$ billion to $\$ 331$ billion for society depending on the treatment duration. Similarly, when compared with baseline, the new targeted therapy scenario extends life expectancy by 0.4 years with a negative social value of $\$ 1,715$ per patient (see table). These results were robust to sensitivity tests on quality-of-life gains and the value of quality-adjusted life-years.

Conclusions: Nivolumab may provide significant survival increases for patients with aRCC and add positive social value, even under the conservative assumption of 2 years' treatment duration to attain full benefit.

\section{2}

\section{Genomic Heterogeneity and the Small Renal Mass}

Ueno, Daiki (Yale School of Medicine, New Haven, CT, United States); Xuoquan, Xie; Boeke, Marta (Yale University, United States); Syed, Jamil (Yale School of Medicine, New Haven, United States); Nguyen, Kevin; McGillivray, Patrick; Adeniran, Adebowale; Humphrey, Peter; Kluger, Yuval; Lu, Zhonzhi (Yale School of Medicine, New Haven, CT, United States); Shuch, Brian (Yale School of Medicine Smilow Cancer Center, New Haven, CT, United States)

Intro: Tumor heterogeneity may represent a barrier to pre-operative genomic characterization by needle biopsy in renal cell carcinoma (RCC). We evaluate heterogeneity in resected small renal masses (SRM) to provide insights into the limitations of pre-treatment biopsy.

Table: Results (difference vs baseline scenario)

\begin{tabular}{lccc}
\hline Scenario & Additional life-years & Social value per patient & Total social value (\$ billion) \\
\hline Received new targeted therapy for 1 year & 0.4 & $-\$ 1,715$ & $-\$ 17.17$ \\
Received second-line nivolumab for 1 year & 1.9 & $\$ 33,108$ & $\$ 331.47$ \\
Received second-line nivolumab for 2 years & 1.9 & $\$ 18,783$ & $\$ 188.06$ \\
\hline
\end{tabular}


Materials and Methods: 23 small (cT1a) and 24 large (cT2+) clear cell RCC had $3+$ regions sampled $>1 \mathrm{~cm}$ apart at time of nephrectomy. Copy number variation $(\mathrm{CNV})$ of driver alterations was assessed using an Illumina Human CytoSNP12 array. Gene expression analysis was performed with a custom Nanostring digital RT-PCR array to characterize previously described clear cell $\mathrm{A}$ and $\mathrm{B}(\mathrm{ccA} / \mathrm{ccB})$ profile and Cell Cycle Progression (CCP) score.

Results: Large tumors more frequently had losses of $14 \mathrm{q} 24,2 \mathrm{q} 37$, and 9p21 ( $<00.05)$. Total CNVs were much less frequent in small tumors (median 2.5 vs 6.5, $\mathrm{p}=0.006$ ). Subclonal CNV events were less common in small tumors (median 0 vs $3, p=0.002$ ). Significant gene expression heterogeneity was observed for both $\mathrm{CCP}$ and $\mathrm{ccA} / \mathrm{B}$ classifications. Larger tumors had significantly more variance in CCP scores $(p=0.026)$. ccA/B scores differed between small and large tumors with mixed ccA/B tumors occurring more frequently in the larger tumors $(p=0.048)$. Analysis of 5 mixed tumors that had $\mathrm{CNV}$ events demonstrated the more aggressive B phenotype had greater $\mathrm{CNV}$ events (median 7 vs 2 , $\mathrm{p}=0.011$ ).

Conclusions: We present the largest cohort of multiregion sampling in clear cell RCC. Small renal tumors have much less genomic complexity and less subclonal events. We demonstrate that $\mathrm{ccA} / \mathrm{ccB}$ profiles can vary within the primary tumor, however this is much less frequent in small renal tumors. Our findings support an ongoing small renal mass trial where pre-treatment genomic characterization is performed based on a single biopsy. However, single biopsies for large tumors might be less useful due to greater heterogeneity.

\section{3}

\section{Germline Mutations in Cancer- Susceptibility Genes in Patients with Advanced Renal Cell Carcinoma}
Maria I. Carlo ${ }^{1 \wedge}$, Semanti Mukherjee ${ }^{2 \wedge}$, Diana Mandelker ${ }^{3}$, Joseph $\mathrm{Vijai}^{2}$, Yelena $\mathrm{Kemel}^{2}$, Liying Zhang $^{3}$, Kuo-Cheng Huan ${ }^{3}$, Almedina Redzematovic ${ }^{1}$, Devyn T. Coskey ${ }^{1}$, Nisha Pradhan ${ }^{1}$, Angela G. Arnold ${ }^{1}$, A. Ari Hakimi ${ }^{4}$, Ying-Bei Chen ${ }^{3}$, Jonathan A. Coleman ${ }^{4}$, David M. Hyman ${ }^{1}$, Mark Ladanyi ${ }^{3}$, Karen A. Cadoo ${ }^{1}$, Michael F. Walsh ${ }^{1}$, Zsofia K. Stadler ${ }^{1}$, Chung-Han Lee ${ }^{1}$, Darren R. Feldman ${ }^{1}$, Martin H. Voss ${ }^{1}$, Mark Robson ${ }^{1}$, Robert J. Motzer ${ }^{1 \#}$, Kenneth Offit ${ }^{1,2, \#}$
${ }^{1}$ Department of Medicine, Memorial Sloan Kettering Cancer Center, New York, NY;
${ }^{2}$ Sloan Kettering Institute, New York, NY;
${ }^{4}$ Department of Surgery, Memorial Sloan Kettering Cancer Center, New York, NY
${ }^{3}$ Department of Pathology, Memorial Sloan Kettering Cancer, New York, NY;

Purpose: Identification of patients with hereditary renal cell carcinoma (RCC) is important for cancer screening and potentially treatment. The prevalence

Figure 1. Frequency and Distribution of Pathogenic Germline Mutations

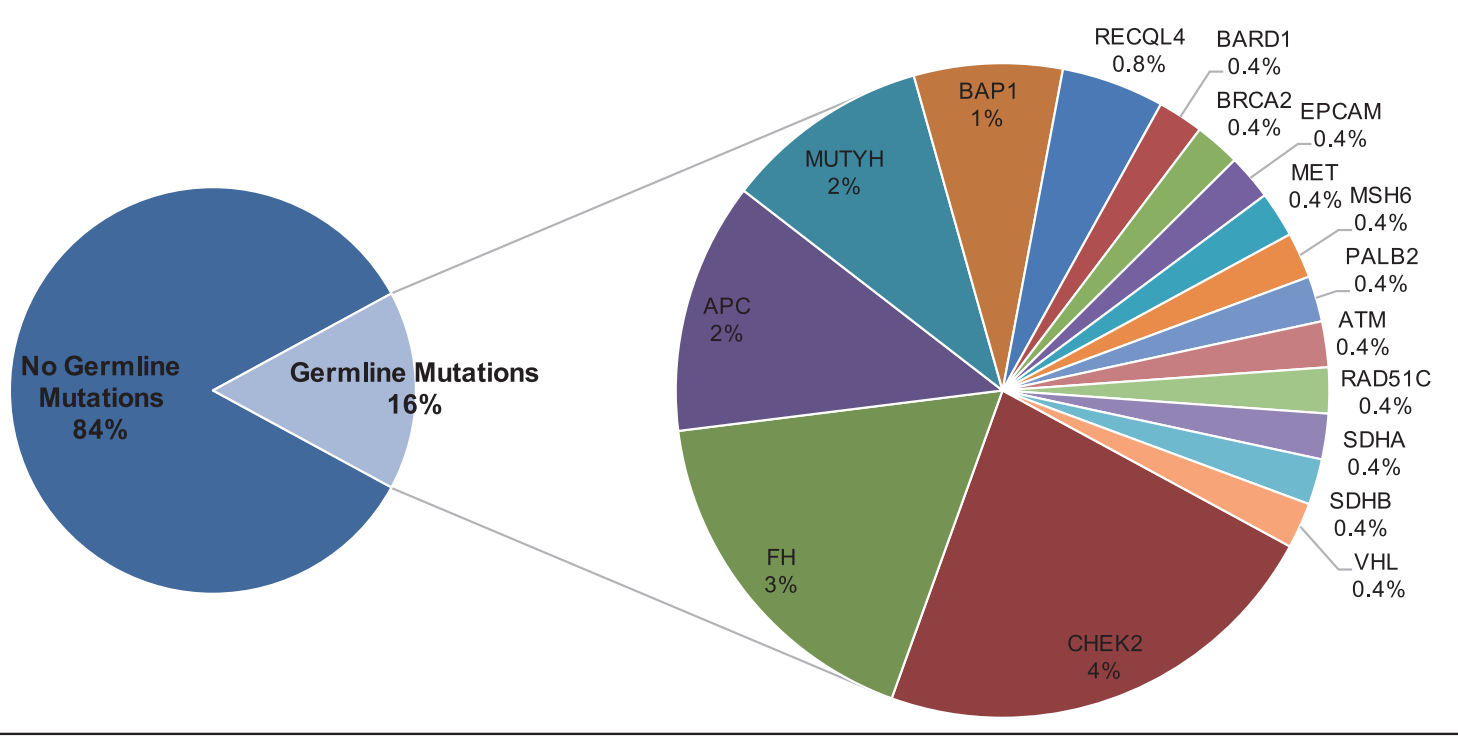


Figure 2. Somatic mutations and loss of heterozygosity in patients with germline mutations

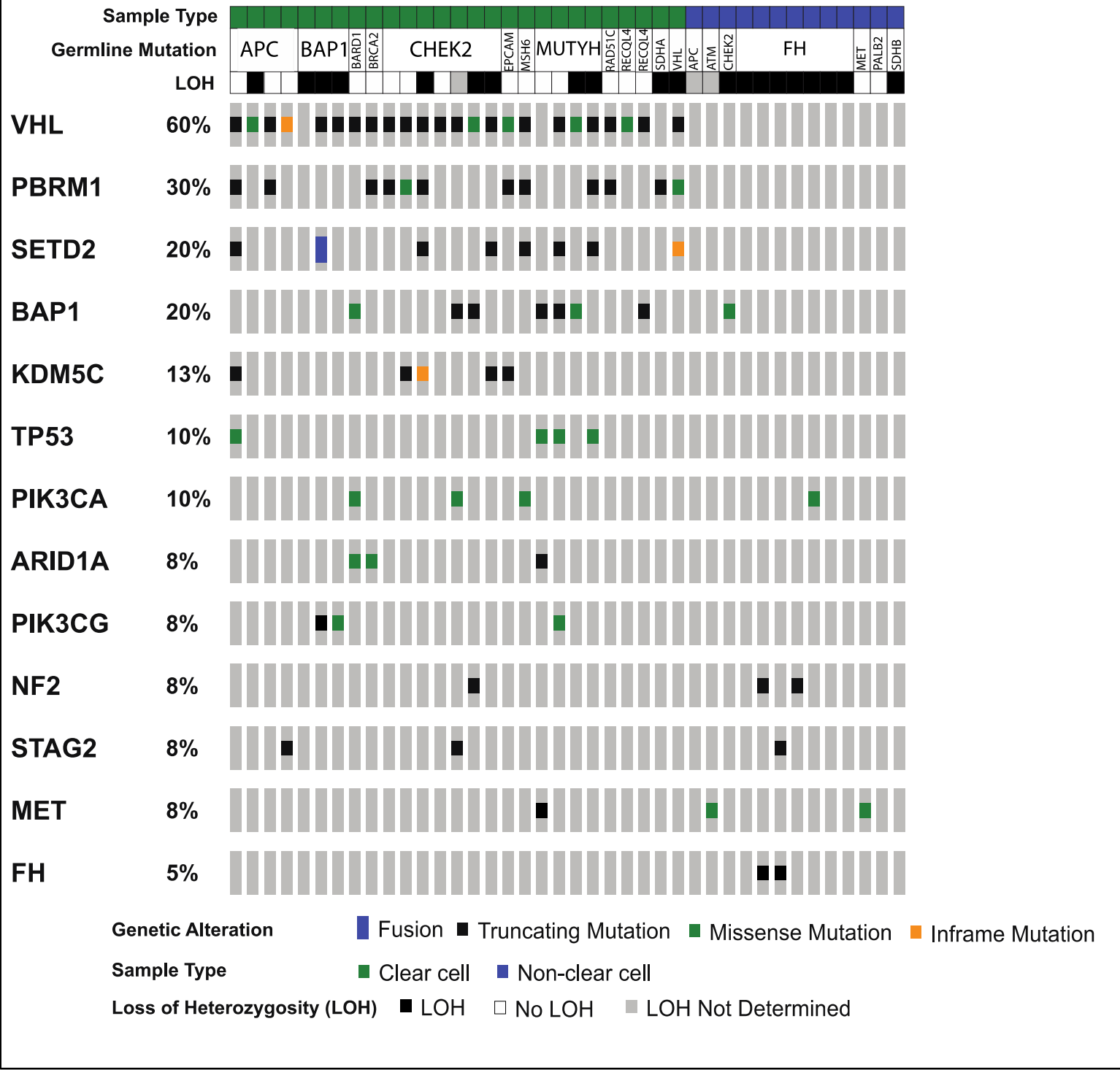

of cancer-associated germline mutations in patients with advanced RCC, and the phenotypes associated with rare mutations, is unknown.

Patients and Methods: Patients with advanced RCC (stage III/IV) who consented to disclosure of germline results as part of a paired tumor-normal gene panel were included $(\mathrm{n}=254)$. Seventy-six genes associated with cancer predisposition were analyzed. Clinical data was obtained from patient questionnaires and medical records, including age of onset, tumor histologic subtype, and tumor multifocality. Published guidelines from the American Col- lege of Medical Genetics (ACMG) were used to determine if patients would have met criteria for genetic testing. A germline mutation was considered "missed" if it was detected by sequencing, but would not have been detected by testing based on application of published guidelines.

Results: Deleterious mutations were identified in $16 \%$ of patients; $5.5 \%$ had mutations in RCC-associated genes ( 7 in $F H, 3$ in $B A P 1$, and 1 each in $V H L$, $M E T, S D H A$, and $S D H B$ ) (Fig. 1). Overall, the most frequent mutations were: CHEK2 (n=9), $F H(\mathrm{n}=7)$, MUTYH $(\mathrm{n}=4)$ and APC $(\mathrm{n}=5)$. Of genes not tradi- 
tionally associated with RCC, CHEK2 was overrepresented in cases compared to the general population, with an odds ratio of RCC of 3.0 $(\mathrm{CI}=1.3-5.8 ; \mathrm{p}=0.003)$. Paired analysis of somatic mutations identified loss of heterozygosity (LOH) in the tumor in over $50 \%$ of patients with CHEK2 germline mutations, supporting its role in the pathogenicity of the tumor (Fig. 2). Non-clear cell histology was associated with presence of RCC-associated gene mutation ( $p=0.001)$, but young age of onset nor family history were not. Applying the ACMG criteria for genetic testing referral, $39 \%$ of all patients would have met criteria for referral, but $36 \%$ of patients with germline mutations in RCC-associated genes would still have been missed.

Conclusion: Among patients with advanced RCC unselected for risk factors for hereditary syndromes, $16 \%$ had germline mutations, the majority in genes not traditionally related with RCC. Histology was associated with presence of RCC-associated germline mutations, but several traditional risk factors were not. CHEK2 mutations were associated with $\mathrm{RCC}$ risk, and could be included in RCC multi-gene panels. Current referral criteria for genetic testing, although broad, did not identify a substantial portion of patients with mutations and should be modified.

\section{4}

\section{IMmotion150: Novel Radiological} Endpoints and Updated Data From a Randomized Phase II Trial Investigating Atezolizumab With or Without Bevacizumab vs Sunitinib in Untreated Metastatic Renal Cell

\section{Carcinoma}

Pal, Sumanta (City of Hope Comprehensive Cancer Center, Duarte, CA, United States); Powles, Thomas (Barts Health NHS Trust - St Bartholomew's Hospital, London, United Kingdom); McDermott, David (Beth Israel Deaconess Medical Center, Boston, MA, United States); Rini, Brian (Cleveland Clinic, Cleveland, $\mathrm{OH}$, United States); Motzer, Robert (Memorial Sloan Kettering Cancer Center, New York, NY, United States); Atkins, Michael (Georgetown Lombardi Comprehensive Cancer Center, Washington, DC, United States); Fong, Lawrence (University of California, San Francisco School of Medicine, San Francisco, CA, United States); Joseph, Richard (Mayo Clinic Hospital, Jacksonville, FL, United States); Ravaud, Alain (CHU Hopitaux de
Bordeaux - Hôpital Saint-André, BORDEAUX, France); Bracarda, Sergio (Ospedale San Donato, Firenze, Italy); Suarez, Cristina (Vall d'Hebron Institute of Oncology, Barcelona, Spain); Maio, Michele (Azienda Ospedaliera Universitaria Senese, Centro di Immuno-Oncologia, Siena, Italy); Gore, Martin (Royal Marsden Hospital, London, United Kingdom); Grunwald, Viktor (Medizinische Hochschule, Zentrum Innere Medizin, Abt Hämatologie u Onkologie, Hanover, Germany); Staehler, Michael (Klinikum d.Universität München Campus Großhadern, Munich, Germany); Qiu, Jiaheng (Genentech, Inc., South San Francisco, CA, United States); Thobhani, Alpa (F. Hoffman-LaRoche Ltd, Welwyn Garden City, United Kingdom); Huseni, Mahrukh; Schiff, Christina (Genentech, Inc., South San Francisco, CA, United States); Escudier, Bernard (Gustave Roussy, Villejuif Cedex, France)

Background: Immunotherapy and VEGF-targeting therapies improve mRCC outcomes; however, new agents and combinations are needed because immune escape and/or resistance often develops. Here, we prospectively explore novel efficacy endpoints and report updated data from IMmotion 150 (NCT01984242), a Ph II trial of atezolizumab (antiPD-L1) with or without bevacizumab (anti-VEGF) vs sunitinib (TKI) in first-line mRCC.

Methods: Treatment-naive mRCC patients were randomized to atezolizumab $1200 \mathrm{mg}$ IV q3w + bevacizumab $15 \mathrm{mg} / \mathrm{kg}$ IV q3w, atezolizumab 1200 mg IV q3w alone or sunitinib $50 \mathrm{mg}$ PO QD 4 wk on/2 wk off. PD-L1 expression was scored on tumor-infiltrating immune cells (IC; VENTANA SP142 IHC assay). Coprimary endpoints were independent review facility (IRF)-assessed PFS (RECIST v1.1) in ITT and PD-L1+ ( $\geq 1 \%$ IC) patients. Other endpoints included investigator (INV)-assessed PFS by RECIST v1.1 and immune-modified RECIST (imRECIST) and patient-reported outcomes (PROs).

Results: After a median 25.7 mo of follow-up, results remained consistent with the primary analysis (median follow-up, $20.7 \mathrm{mo}$ ), showing clinically meaningful benefit in IRF- and INV-PFS with atezolizumab + bevacizumab vs sunitinib in PD-L1+ patients (RECIST v1.1; Table). The INV-PFS (imRECIST) HR for atezolizumab + bevacizumab vs sunitinib was 0.78 in ITT patients and 0.47 in PD$\mathrm{L} 1+$ patients (Table). Safety of atezolizumab + bevacizumab was consistent with the known safety profile of each agent alone; further follow-up showed no new safety signals. PROs will be presented. 


\begin{tabular}{|c|c|c|c|c|c|c|}
\hline & \multicolumn{3}{|c|}{ ITT } & \multicolumn{3}{|c|}{$\begin{array}{c}\text { PD-L1 Expression on } \geq 1 \% \text { of IC } \\
\text { (PD-L1+) }\end{array}$} \\
\hline & $\begin{array}{c}\text { Sun } \\
\mathbf{n}=101\end{array}$ & $\begin{array}{c}\text { Atezo } \\
\mathrm{n}=\mathbf{1 0 3}\end{array}$ & $\begin{array}{c}\text { Atezo }+ \text { Bev } \\
\mathbf{n}=\mathbf{1 0 1}\end{array}$ & $\begin{array}{c}\text { Sun } \\
\mathrm{n}=\mathbf{6 0}\end{array}$ & $\begin{array}{l}\text { Atezo } \\
\mathrm{n}=54\end{array}$ & $\begin{array}{l}\text { Atezo }+ \text { Bev } \\
\quad \mathbf{n}=\mathbf{5 0}\end{array}$ \\
\hline $\begin{array}{l}\text { Median PFS } \\
\text { (IRF, RECIST v1.1), mo } \\
(95 \% \text { CI) }\end{array}$ & $\begin{array}{c}8.1 \\
(5.7,10.9)\end{array}$ & $\begin{array}{c}6.7 \\
(5.4,13.6)\end{array}$ & $\begin{array}{c}11.6 \\
(8.4,17.3)\end{array}$ & $\begin{array}{c}6.4 \\
(3.8,8.7)\end{array}$ & $\begin{array}{c}5.4 \\
(3.0,11.1)\end{array}$ & $\begin{array}{c}12.1 \\
(8.2,22.9)\end{array}$ \\
\hline $\begin{array}{l}\text { Stratified HR vs sunitinib } \\
(95 \% \mathrm{CI})\end{array}$ & - & $\begin{array}{c}1.04 \\
(0.73,1.48)\end{array}$ & $\begin{array}{c}0.95 \\
(0.67,1.34)\end{array}$ & - & $\begin{array}{c}0.97 \\
(0.61,1.54)\end{array}$ & $\begin{array}{c}0.65 \\
(0.40,1.05)\end{array}$ \\
\hline $\begin{array}{l}\text { Median PFS } \\
(\text { INV, RECIST v1.1), mo } \\
(95 \% \text { CI })\end{array}$ & $\begin{array}{c}7.8 \\
(5.7,9.8)\end{array}$ & $\begin{array}{c}5.5 \\
(3.0,8.3)\end{array}$ & $\begin{array}{c}11.0 \\
(8.2,13.5)\end{array}$ & $\begin{array}{c}6.8 \\
(5.4,11.2)\end{array}$ & $\begin{array}{c}5.5 \\
(3.0,10.9)\end{array}$ & $\begin{array}{c}11.1 \\
(8.1,16.7)\end{array}$ \\
\hline $\begin{array}{l}\text { Stratified HR vs sunitinib } \\
(95 \% \mathrm{CI})\end{array}$ & - & $\begin{array}{c}1.13 \\
(0.82,1.55)\end{array}$ & $\begin{array}{c}0.88 \\
(0.64,1.22)\end{array}$ & - & $\begin{array}{c}0.96 \\
(0.63,1.46)\end{array}$ & $\begin{array}{c}0.60 \\
(0.38,0.94)\end{array}$ \\
\hline $\begin{array}{l}\text { Median PFS } \\
\left(\text { INV, imRECIST }^{\mathrm{a}}\right), \text { mo } \\
(95 \% \mathrm{CI})\end{array}$ & $\begin{array}{c}9.9 \\
(7.0,14.1)\end{array}$ & $\begin{array}{c}8.5 \\
(7.9,13.6)\end{array}$ & $\begin{array}{c}17.3 \\
(11.6,24.9)\end{array}$ & $\begin{array}{c}8.4 \\
(5.8,11.3)\end{array}$ & $\begin{array}{c}10.9 \\
(5.4,14.0)\end{array}$ & $\begin{array}{c}21.9 \\
(11.1,27.6)\end{array}$ \\
\hline $\begin{array}{l}\text { Stratified HR } \\
\text { vs sunitinib }(95 \% \mathrm{CI})\end{array}$ & - & $\begin{array}{c}1.05 \\
(0.74,1.49)\end{array}$ & $\begin{array}{c}0.78 \\
(0.55,1.11)\end{array}$ & - & $\begin{array}{c}0.87 \\
(0.55,1.39)\end{array}$ & $\begin{array}{c}0.47 \\
(0.29,0.78)\end{array}$ \\
\hline
\end{tabular}

a imRECIST criteria are based on RECIST v1.1 and include adaptations for response patterns observed with cancer immunotherapy (Mazieres et al. ASCO 2016).

Data cutoff: April 2017

Conclusions: Updated efficacy (RECIST v1.1) confirmed the encouraging activity of atezolizumab + bevacizumab in PD-L1+ first-line mRCC, with no new safety signals. Data per imRECIST, compared with RECIST v1.1, showed benefit of atezolizumab + bevacizumab in PD-L1+ and ITT patients and may contribute to our understanding of the clinical activity of cancer immunotherapy in mRCC. The clinical benefit of atezolizumab + bevacizumab vs sunitinib will be further evaluated in the ongoing Ph III study, IMmotion151 (NCT02420821).

\section{5}

\section{Immune Cell Infiltration within Differing Renal Cell Carcinoma Primary Histologies: Preliminary Report}

Kyle A. Blum ${ }^{1}$, Renzo G DiNatale ${ }^{1}$, Alejandro Sanchez ${ }^{1}$, Nirmal T. John ${ }^{1}$, Mazyar Ghanaat ${ }^{1}$, Ming Liu ${ }^{1}$, Briana G. Nixon ${ }^{1}$, Paul Russo', Victor Reuter ${ }^{1}$, Ming O. Li ${ }^{1}$, A. Ari Hakimi $^{1}$

\section{${ }^{I}$ Memorial Sloan Kettering Cancer Center, New York, NY}

Funding: Ruth L. Kirschstein Research Service Award T32CA082088 (A.S).

Introduction and objectives: The amount of tumorinfiltrating cells in renal cell carcinoma (RCC) has been associated with response to immunotherapy and prognosis. In this exploratory study we aim to characterize immune cell penetration within the RCC tumor microenvironment based on primary histologies of clear cell RCC (ccRCC), papillary RCC (pRCC), chromophobe RCC (chRCC) and unclassified RCC (uRCC).

Methods: Tumor and normal kidney tissue from 56 patients who underwent surgical excision from 12/2015-7/2017 were prospectively collected for analysis. Forty-five patients had ccRCC, 2 pRCC, 5 chRCC, and 4 uRCC. Immune cell phenotyping was performed using staining single-cell suspensions followed by flow cytometry. The mean differences in immune cell populations within the matched tumor-normal samples were analyzed by primary histology. Cell types analyzed included CD45+ cells (general marker of immune cells), CD3 $+\mathrm{T}$ cells, CD4+ T cells, CD8+ T cells, and CD4CD25+ Tregs. $\mathrm{P}$-values were calculated by comparing ccRCC to the other primary histologies using two-sample Ttests with a pre-specified rejection level of 0.05 .

Results: Median age of this cohort was 59 years, with $69.6 \%$ male and $21(37.5 \%)$ presenting with metastatic disease. Median tumor pathological size was $8.6 \mathrm{~cm} \mathrm{(2.9-18.1),} \mathrm{and} 41$ (73.2\%) had pT3pT4 disease. An enrichment of CD45+ cells were identified in $82.14(\%)$ of tumors compared to their corresponding normal tissue. The mean difference in 
CD45+ cells between matched samples in ccRCC, chRCC, pRCC, and uRCC was $33.97(\mathrm{p}<0.001)$, $13.71(p<0.001), 13.91(p=0.27)$, and $-0.73(p=0.97)$, respectively, Figure 1. Interestingly, a mean difference of $-0.73(p=0.97)$ in uRCC suggests fewer immune cells were found within the tumor than normal kidney. When comparing the mean differences in CD45+ across all histologies, we found a significant difference between immune infiltration in ccRCC and chRCC of 20.3 (95\%CI 9.8-30.6, $\mathrm{p}=0.0004)$, figure 1 . The observed trend of elevated immune cell infiltration across histologies was not limited to a specific cell type as all types analyzed (CD45 $(p<0.001), C D 3(p<0.001), C D 4(p<0.0029), C D 8$ $(\mathrm{p}<0.001), \mathrm{CD} 4 \mathrm{CD} 25(\mathrm{p}<0.001))$ experienced a universal increase.

Conclusions: Our exploratory study showed a higher proportion of immune cells (CD45+) in tumor tissue compared to normal kidney especially in ccRCC tumor samples. Interestingly among the 4 uRCC patients, fewer immune cells were found in the tumor relative to normal kidney perhaps suggestive of immune exclusion. Elucidating these immune cell infiltration signatures may correlate with clinical outcomes and help identify those likely to benefit from immunotherapy. Further refinement of immune profile differences and validation of these findings in a larger cohort is currently ongoing.

Figure 1: Mean Change in CD45+ Cell Infiltrate in RCC Primary Histologies.

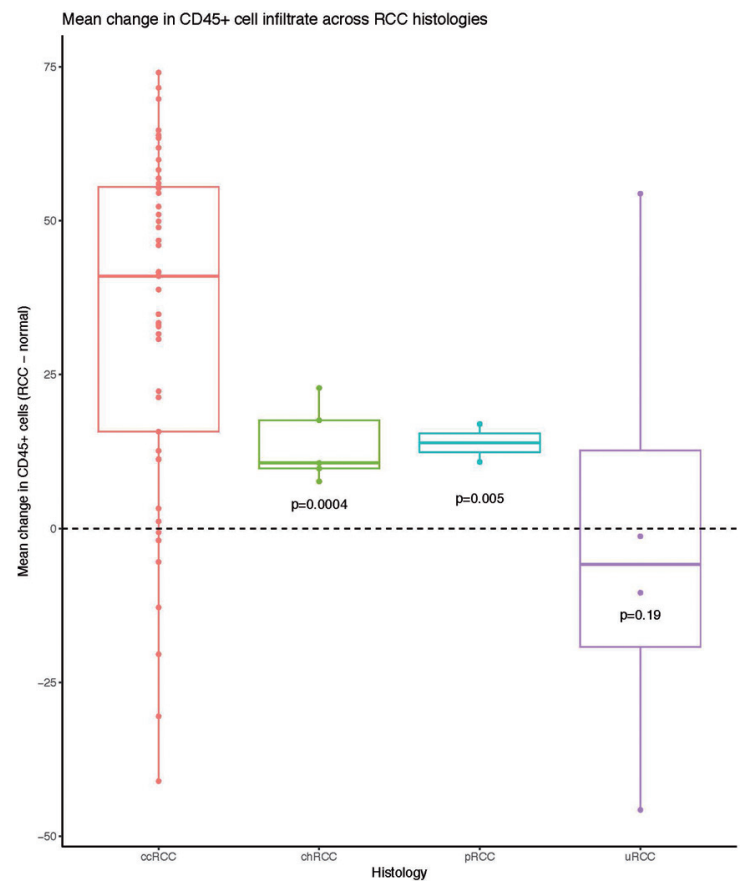

\section{Impact of antibiotics on outcome in patients with metastatic renal cell carcinoma treated with immune checkpoint inhibitors}

Derosa, Lisa (Gustave Roussy Cancer Campus, Villejuif, France); Routy, Bertrand; Enot, David; Fidelle, Marine (Gustave Roussy Cancer Campus, Villejuif, France); Gubet, Anne-Gaelle (Gustave Roussy Cancer Campus, Villejuif, France); Goldwasser, Francois (Cochin Hospital, Paris, Paris, France); Zitvogel, Laurence; Loriot, Yohann; Albiges, Laurence; Escudier, Bernard (Gustave Roussy Cancer Campus, Villejuif, France)

Background: The clinical relevance of gut microbiota composition particularly in the success of immune checkpoint inhibitors (ICI) emerged as a potential novel biomarker. Use of antibiotics (ATB) alters the gut microbiota composition and decreases bacterial diversity. However, the interaction between ATB and ICI has not been extensively investigated in patients (pts). Our study evaluated the effect of ATB in cancer pts treated with PD-1/PD-L1 inhibitors.

Methods: We conducted a retrospective analysis of pts treated with PD-1/PD-L1 inhibitors for advanced Renal Cell Carcinoma (RCC) and data on ATB use were collected. ATB group was defined as patients prescribed with ATB in the window period of 2 months before or 1 month after the first administration of ICI. Primary end point was best overall response assessed centrally by RECIST 1.1. Progression free survival (PFS) and overall survival (OS) were defined as secondary end points. Statistical analyses were performed using the Kaplan-Meier method and Cox regression model.

Results: After a median follow-up of 13.8 months, among 121 pts included, 34 (28\%) received ATB (mostly beta-lactamases and fluoroquinolones). ATB were generally prescribed per os $(94 \%)$ and the rate of hospitalization was less than $15 \%$. Considering best response ATB group had more progressive disease compared to no ATB ( $50 \%$ vs $19 \%, p<0.01)$. PFS and OS were significantly shorter between ATB and no ATB group (4.3 versus 7.4 months, $p=0.02$, and 23.4 versus 31.1 months, $p=0.03$, respectively). According to treatment, ATB group translated into decrease PFS and OS, in patients treated with PD-1 inhibitors (n:101). The negative impact of ATB was maintained for PFS after multivariate analyses adjusting for risk factors. 
Conclusions: Among patients with RCC treated with ICI, ATB had a deleterious effect on best overall response, PFS and OS. Interventions to determine gut microbiota composition before ICI initiation opens an unexpected strategy in oncology. In addition, validation cohorts are ongoing, such us in lung cancer (manuscript in preparation with MSKCC Lung Cancer Group).

\section{7}

\section{Integrated biomarker analysis for $\mathbf{4 1 2}$ renal cell cancer ( $\mathrm{RCC}$ ) patients (pts) treated on the phase 3 COMPARZ trial: Correlating common mutation events in PBRM1 and BAP1 with angiogenesis expression signatures and outcomes on tyrosine kinase inhibitor (TKI)}

\footnotetext{
Martin Henner Voss ${ }^{1}$, Fengshen $\mathrm{Kuo}^{1}$, Mahtab Marker ${ }^{2}$, Yuan Cheng ${ }^{2}$, Parul Patel ${ }^{2}$, Almedina Redzematovic ${ }^{1}$, Nadeem Riaz ${ }^{1}$, Timothy A. Chan ${ }^{1}$, Toni K. Choueiri ${ }^{3}$, James Hsieh ${ }^{4}$, Robert J. Motzer ${ }^{1}$, A. Ari Hakimi ${ }^{1}$

${ }^{1}$ Memorial Sloan-Kettering Cancer Center, New York, NY; ${ }^{2}$ Novartis Pharmaceuticals Corporation, East Hanover, NJ; ${ }^{3}$ Dana-Farber Cancer Institute and Brigham and Women's Hospital, Boston, MA; ${ }^{4}$ Dept. of Medicine, Washington University School of Medicine, St. Louis, $M O$
}

Background: In RCC biology mutations in PBRM1 and BAP1are largely non-overlapping and collectively affect $>50 \%$ of pts. How and through which mechanism they influence disease kinetics is poorly understood. Sunitinib and pazopanib inhibit angiogenesis, a key driver in RCC. We analyzed mutation status and gene expression signatures in a large cohort of pts receiving first-line sunitinib or pazopanib on the COMPARZ trial

Methods: RNA and DNA were extracted from archival tissue. PBRM1 and BAP1mutation status was determined via a custom exon-targeted platform. Transcriptome analysis was done using Affymetrix Gene Chip HTA 2.0. We computed a 43 gene angiogenesis expression score with previously reported dynamic response to VEGF-directed therapy in xenograft models (Masiero, Cancer Cell 2013). DNA and RNA findings were correlated with clinical outcomes using parametric and non-parametric tests
Results: 412 pts contributed tumor RNA, 377 pts DNA; 362 pts both. PBRM1 and BAP1 were mutated (MT) in $44 \%$ and $15 \%$ of pts, respectively. Presence of PBRM1 mutations correlated with superior PFS $(\mathrm{p}=0.008)$ and $\mathrm{OS}(\mathrm{p}=0.004)$ on log-rank test, and PBRM1 mutation rate was higher in pts with objective response than those with progression (Fisher's Exact, $\mathrm{p}=0.012$ ). In contrast, pts with MT BAP1 had inferior OS compared to those whose were wild type (WT) (log-rank, $\mathrm{p}=0.012$ ). Across all 412 pts angiogenesis score associated favorably with outcome on uni and multivariate analyses (Cox proportional hazard regression, OS $\mathrm{p}<0.001$ and PFS $p<0.005$ ); scores were higher in 123 pts with objective response than $81 \mathrm{pts}$ with progression as best response (Mann-Whitney, $\mathrm{p}=0.009$ ). Angiogenesis scores were higher in PBRM1 MT vs WT patients (Mann-Whitney, $\mathrm{p}<0.001$ ), but lower in BAP1 MT vs WT patients $(\mathrm{p}<0.001)$

Conclusions: PBRM1 and BAP1 mutations appear to have opposite effects in advanced RCC. Loss of PBRM1 enhances the pro-angiogenic microenvironment of RCC with favorable effects on response to TKI; BAP1 loss associates with decreased angiogenic signaling and adverse outcome to TKI. Clinical trial information: NCT00720941

Reused with permission from the American Society of Clinical Oncology (ASCO). This abstract was accepted and previously presented at the 2017 ASCO Annual meeting. All rights reserved

28

Long-term response and time to response to pazopanib (PAZ) and sunitinib (SUN) in metastatic renal cell carcinoma (mRCC): COMPARZ subanalysis

Tannir, Nizar M. (MD Anderson Cancer Center, Houston, TX, United States); Porta, Camillo (IRCCS San Matteo University Hospital Foundation, Pavia, PV, Italy); Grünwald, Viktor (Hannover Medical School, Hannover, GER, Germany); Choueiri, Toni K. (Dana Farber Cancer Institute, Boston, MA, United States); Ahmad, Qasim I.; Carrasco-Alfonso, Marlene J.; Dezzani, Luca; Han, Jackie (Novartis Pharmaceuticals Corporation, East Hanover, NJ, United States); Sternberg, Cora N (San Camillo and Forlanini Hospitals, Roma, RM, Italy) 
Background: COMPARZ (NCT00720941) was a phase 3, randomized, controlled, open label trial that demonstrated comparable efficacy of first line PAZ and SUN, but favorable safety and quality of life profiles for PAZ in 1110 patients with $\mathrm{mRCC}$ (NEJM 2013;369:722). The objectives of this study were to identify patients from COMPARZ who exhibited a long-term response (LTR) to PAZ and SUN, determine time to response, and describe the clinical characteristics of patients who achieved LTR

Methods: Patients in the intention-to-treat population of COMPARZ were analyzed for differences in LTR ( $\geq 10$ months [mos]) as measured by responder rate with either complete response or partial response (CR/PR) and PFS rate, and time to response. We also compared the clinical characteristics between long-term and shorter-term responders within and between each treatment arm

Results: The overall percentage of long-term responders with $\mathrm{CR} / \mathrm{PR}(\mathrm{PAZ}=14 \%, \mathrm{SUN}=13 \%)$ and PFS (PAZ $=31.4 \%$, SUN $=33.6 \%)$ in the PAZ and SUN groups were similar. This similarity was observed regardless of the cutoff for long-term duration of response. However, a shorter time to achieve $\mathrm{CR} / \mathrm{PR}$ was observed in the overall population with PAZ (11.9 weeks [95\% CI, 11.3-12.1] vs 17.4 weeks; [95\% CI, 12.7-18.0]). Analysis conducted to identify baseline clinical characteristics that may be related to LTR will be reported

Conclusions: These exploratory subanalysis in long-term responders support the overall efficacy results with PAZ and SUN, which were reported in the COMPARZ trial. However, the results here demonstrate that the time to response was shorter with PAZ versus SUN. Clinical trial information: NCT00720941

Reused with permission from the American Society of Clinical Oncology (ASCO). This abstract was accepted and previously presented at the 2017 ASCO Annual meeting. All rights reserved

\section{9}

\section{Physician Treatment Selection in the Prospective Metastatic Renal Cell Cancer (MaRCC) Registry}

Costello, Brian A. (Mayo Clinic, Rochester, MN, United States); Bhavsar, Nrupen A.; Harrison, Michael R. (Duke University Medical Center, Durham, NC, United States);
Zakharia, Yousef (University of Iowa Hospitals and Clinics, Iowa City, IA, United States); Vaishampayan, Ulka (Karmanos Cancer Institute, Detroit, MI, United States); Pal, Sumanta K. (City of Hope, Duarte, CA, United States); Jim, Heather; Fishman, Mayer N. (Moffitt Cancer Center, Tampa, FL, United States); Molina, Ana M. (Weill Cornell Medicine, New York, NY, United States); Kyriakopoulos, Christos (University of Wisconsin, Madison, WI, United States); Tsao, Che-Kai (Tisch Cancer Institute, Mount Sinai Medical Center, New York, NY, United States); Appleman, Leonard J. (UPMC Cancer Pavilion, Pittsburgh, PA, United States); Gartrell, Benjamin A. (Montefiore Hospital and Medical Center, Bronx, NY, United States); Hussain, Arif (University of Maryland, Baltimore, MD, United States), Stadler, Walter M. (The University of Chicago, Chicago, IL, United States); Agarwal, Neeraj (Huntsman Cancer Institute, Salt Lake City, UT, United States); Pachynski, Russell (Washington University School of Medicine, St Louis, MO, United States); Hutson, Thomas E. (Baylor Sammons Cancer Center-Texas Oncology, Dallas, TX, United States); Hammers, Hans J. (UT Southwestern, Dallas, TX, United States); Ryan, Christopher W. (Oregon Health and Science University, Portland, OR, United States); Mardekian, Jack; Singh, Kanwarjit; Borham, Azah (Pfizer Inc, New York, NY, United States); George, Daniel J. (Duke University Medical Center, Durham, NC, United States)

Background: Characteristics of patients enrolled in clinical trials of metastatic renal cell carcinoma $(\mathrm{mRCC})$ can often differ from those in the real world setting, resulting in potential bias in the literature. The MaRCC Registry is designed to survey questions not asked in trials, such as what practicing physicians choose as first-line therapy among many available options, and why physicians make certain management decisions. This analysis describes physician treatment selection of first line therapy and reasons for treatment selection in 502 real world patients enrolled in a multicenter, prospective, observational registry.

Methods: The MaRCC Registry enrolled 502 patients from 46 US academic (ACAD) $(\mathrm{N}=20)$ and community $(\mathrm{N}=26)$ sites with $\mathrm{mRCC}$ and no prior systemic therapy for metastatic disease. Key endpoints included descriptive treatment characteristics (e.g. treatment agents, sequence, duration, reasons for therapy choice and discontinuation), treatment effectiveness (e.g. overall response rate, progression free survival (PFS), overall survival (OS)), quality of life as collected via patient reported outcomes, medication adherence, ability to work, hospitalizations, and emergency department visits. 
Here we describe preliminary results from the physician surveys conducted at the time of study enrollment.

Results: At data cut-off of August 4, 2017, 502 patients had been accrued: median age 63 (Q1-3 range, 56-70); 70\% male; 75\% ACAD; 78\% clear cell histology; and 33\% stage IV at diagnosis. Initial management decision was: $59 \%$ (294) went directly on systemic therapy (ST), whereas in 41\% (208) systemic therapy was deferred (DST). At time of data cut-off and through a median follow-up of 8.5 months, $73 / 208(35 \%)$ of the DST patients started ST. For all patients treated with ST (367) in the registry, first line treatments included pazopanib (45\%), sunitinib (21\%), clinical trial (19\%), and high-dose interleukin-2 (5\%). The most common categories for therapy choice as selected by providers were likelihood of clinical benefit (61\%) followed by patient characteristics (25\%). Within the clinical benefit category, the most common reason was OS/PFS (37\%), followed by likelihood of tumor regression (27\%). Within the patient characteristic category the most common reasons included prognostic factors $(12 \%)$ or performance status/frailty $(7 \%)$. In the DST group, ECOG 0 was seen in $46 \%$ and in ST, $35 \%$. For first line ST, the mean and median starting pazopanib dose was $684 \mathrm{mg}$ and $800 \mathrm{mg}$ respectively, with $114 / 164$ patients $(69.5 \%)$ starting at full dose $(800 \mathrm{mg})$. The mean and median starting sunitinib dose was $49 \mathrm{mg}$ and $50 \mathrm{mg}$ respectively, with $70 / 79$ patients $(88.6 \%)$ starting at full dose $(50 \mathrm{mg})$.

Conclusions: This is the first prospective report of physician practice patterns in the first line treatment of $\mathrm{mRCC}$ patients in the real world setting. We describe the most common factors driving physician decision making in management of $\mathrm{mRCC}$. Early experience suggests that clinical benefit associated with treatment and patient characteristics were the most common reasons for initial ST selection. Interestingly, side effect profile rarely determined initial ST selection.

\section{0}

\section{Plasma glycosaminoglycan scores in early stage renal cell carcinoma}

Gatto, Francesco (Chalmers University of Technology, Göteborg, Sweden); Hakimi, Abraham (Memorial Sloan Kettering Cancer Center, New York City, United States); Nielsen, Jens (Chalmers University of Technology, Göteborg, Sweden)
Introduction \& objectives: No diagnostic blood biomarker for RCC is currently used in the clinical routine. Using a systems biology approach, we previously developed a score based on circulating glycosaminoglycans (GAGs) that detected metastatic clear cell renal cell carcinoma (RCC) with 92.6\%, $93.7 \%$, and $100 \%$ accuracy vs. healthy subjects using either plasma, urine, or combined measurements in a validation cohort (Gatto et al., 2016, Cell Reports). It is still unknown if this test is accurate in early stage RCC or other RCC histologies. The primary endpoint of this study was the area-under-thecurve (AUC) in the use of plasma GAG scores to detect pre-operative RCC, any stage and any histology, versus healthy individuals.

Materials \& methods: Sample collection was retrospective. Eligibility criteria were: patients with radiographic finding of renal mass; healthy volunteers without any history of malignancy. Participants were enrolled as a consecutive series at the Memorial Sloan Kettering Cancer Center, New York City, United States between 2011 and 2014. Plasma samples were collected in EDTA-coated tubes up to 4 weeks before nephrectomy. A convenience sub-cohort was followed longitudinally and samples were collected during follow-up visits up to 12 months after nephrectomy. A group of samples from healthy subjects was formed using plasma collected among relatives of cancer patients or available in previously stored samples. Laboratory measurements of GAGs was performed using capillary electrophoresis with laser induced fluorescence. Samples were scored according to the previously published formula to compute plasma GAG scores. Pre-specified variations of this formula were: omission of total chondroitin sulfate concentration; omission of heparan sulfate terms; omission of both.

Results: In total, we collected 470 samples from 237 subjects with a radiographic finding of renal mass and 44 samples from as many healthy volunteers. We report interim analysis of GAGs in the 25 stored samples from healthy volunteers and in 195 randomly selected samples from subjects with a renal mass. Of these, 71 were obtained pre-operatively from patients with subsequent pathologic diagnosis of RCC. Fifty had clear cell RCC (25 Stage I, 1 Stage II, 21 Stage III, and 3 Stage IV), while 21 had non-clear cell RCC (14 Stage I, 3 Stage II, 2 Stage III, and 1 Stage IV, 1 NA). The AUC in the detection of RCC, any stage, any histology, versus healthy was 0.866 (95\% CI: 0.796-0.937), 0.883 (CI: 0.814- 
0.954), 0.999 (CI: 0.996-1), and 0.947 (CI: 0.9030.991 ) using, respectively, the original plasma GAG score, or the variations without total chondroitin sulfate concentration, without any heparan sulfate terms, or without either.

Conclusion: Interim analyses revealed that plasma GAG scores have the potential to differentiate samples from RCC versus healthy subjects with remarkable AUC, ranging 0.866 to 0.999 depending on the different formula variations.

\section{1}

\section{Predictive genomic markers of response to VEGF targeted therapy (TT) in metastatic renal cell carcinoma (mRCC): Role of VHL and TP53 mutation, and FLT1 germline variant}

Andrew W. Hahn'1, David M. Gill'1, Dan Albertson², Banumathy Gowrishankar ${ }^{3}$, Joseph Merriman ${ }^{1}$, Archana M. Agarwal ${ }^{2}$, Venkata Thodima ${ }^{3}$, Erik Harrington ${ }^{4}$, Trang $\mathrm{Au}^{4}$, Benjamin L. Maughan ${ }^{1}$, Jane Houldsworth ${ }^{3,5}$, David D. Stenehjem ${ }^{1,4}$, Sumanta K. $\mathrm{Pal}^{6}$, Neeraj Agarwal ${ }^{1}$

${ }^{I}$ Department of Internal Medicine, Division of Medical Oncology, University of Utah Huntsman Cancer Institute, Salt Lake City, UT, USA

${ }^{2}$ Department of Pathology, University of Utah and ARUP Laboratories, Salt Lake City, UT, USA

${ }^{3}$ Cancer Genetics Inc., Rutherford, NJ

${ }^{4}$ Pharmacotherapy Outcomes Research Center (PORC), College of Pharmacy, University of Utah, Salt Lake City,

UT, USA

${ }^{5}$ Department of Pathology, Mount Sinai School of

Medicine, New York City, NY

${ }^{6}$ Department of Medical Oncology \& Experimental

Therapeutics, City of Hope Comprehensive Cancer

Center, Duarte, $C A$
Introduction: In the first-line therapy setting for mRCC, VEGF tyrosine-kinase inhibitors, mTOR inhibitors, and high-dose IL-2 are current standards. Checkpoint inhibitors are expected to garner approval soon. In the absence of head-to-head comparison of these agents, genomic markers of response to therapy are needed to guide therapy selection. The objective of this study was to identify tumor-based genomic markers of response to VEGF TT to optimize treatment selection.

Methods: Targeted sequencing of primary tumors of patients with $\mathrm{mRCC}$ was performed, and tumor genomic aberrations (GAs) were correlated with progression-free survival (PFS) to treatment with first-line VEGF targeted therapies by using KaplanMeier methodology and Cox proportional hazard models. A composite model of all statistically significant GAs predicting PFS in the first line setting was developed.

Results: Mutations in TP53 were associated with inferior PFS on first-line VEGF TT (HR 2.83, 95\% CI 1.05-6.68; $\mathrm{p}=0.023$ ), whereas, VHL mutations were associated with improved PFS (HR 0.40, 95\% CI 0.21-0.78; $\mathrm{p}=0.0042$ ). A trend for inferior PFS was observed with FLT1 C/C variant. A composite model of these 3 GAs was significantly associated with inferior PFS and OS in a dose-dependent manner, when controlling for IMDC risk category in a Cox proportional hazard model (Table).

Conclusion: A composite model of tumor GAs, including TP53 mutation, wild type VHL, and FLT1 $\mathrm{C} / \mathrm{C}$ variant significantly predicted survival outcomes to first-line therapy with VEGF TT in mRCC in a dose-dependent manner.

Table: Cox proportional hazard model for PFS and overall survival by IMDC risk criteria and sum of VHL wildtype, TP53 mutated, and $F L T 1 \mathrm{C} / \mathrm{C}$

\begin{tabular}{lcccc} 
& \multicolumn{2}{c}{ Progression-free Survival } & \multicolumn{2}{c}{ Overall Survival } \\
\cline { 2 - 5 } & Hazard ratio, 95\% CI & Log-Rank & Hazard ratio, 95\% CI & Log-Rank \\
\hline 1 vs. 0 & $1.70(0.81-3.42)$ & 0.15 & $2.37(1.11-4.80)$ & $\mathbf{0 . 0 2 6}$ \\
2 or 3 vs. 1 & $3.50(1.17-9.62)$ & $\mathbf{0 . 0 2 7}$ & $2.27(0.62-6.75)$ & 0.20 \\
2 or 3 vs. 0 & 2 or 3 vs $0: 5.97(2.06-15.36)$ & $\mathbf{0 . 0 0 1 9}$ & $5.37(1.50-15.2)$ & $\mathbf{0 . 0 1 3}$ \\
\hline
\end{tabular}




\section{2}

\section{Productivity, Satisfaction, and Health- Related Quality of Life in Advanced Renal Cell Carcinoma Patients Receiving 2 or More Lines of Treatment: Results from a United Kingdom (UK) chart review}

\author{
Doan, Justin (Bristol-Myers Squibb, Princeton, NJ, \\ United States); Deshpande, Chinmay; Solem, Caitlyn \\ (Pharmerit International, Bethesda, MD, United States); \\ Malcolm, Bill (Bristol-Myers Squibb, Princeton, MD, \\ United States); Macahilig, Cynthia (Medical Data \\ Analytics, Parsippany, NJ, United States); Jiang, Shan \\ (Pharmerit International, Bethesda, MD, United States); \\ Hawkins, Robert (University of Manchester, Manchester, \\ EN, United Kingdom)
}

Background: The humanistic burden associated with advanced renal cell carcinoma (aRCC) in the European population and especially in the United Kingdom (UK) is not well understood. This study aims to describe the work-productivity and quality of life (QOL) among aRCC patients in the UK.

Methods: This retrospective chart review enrolled adults diagnosed with aRCC between 12/23/2016 and 05/26/2017, who received at least 2 lines (L) of systemic therapy for aRCC. Patients were excluded if they were enrolled in a cancer treatment-related clinical trial after aRCC diagnosis. Patients who agreed to participate completed a one-time computer-assisted telephone interview composed of the following instruments: Euroqol EQ-5D-5L (index range: 0 [worst] to 1 [best]; visual analog scale [VAS]: 0 [worst] to 100 [best]), Functional Assessment of Chronic Illness Therapy Measurement System (FACT-G Total; range: 0 [worst] to 108 [best]; FACT-G physical, social, and functional well-being dimensions, range: 0 [worst] to 28 [best]), Functional Assessment of Cancer Therapy-Kidney Symptom Index (FKSI-19 Total; range: 0 [worst] to 76 [best]), Cancer Therapy Satisfaction Questionnaire (CTSQ; range: 0 [worst] to 100 [best]), and Work Productivity and Activity Impairment (WPAI; range: 0 [best] to 100 [worst]).

Results: A total of 65 patients (mean age 61 years, $60 \%$ male, $44 \%$ stage IV) were included from 7 sites. Health-related quality of life as measured by the EQ5D-5L had a mean index of 0.56 (SD 0.37) and VAS of 61.8 (22.7), with over half of patients reporting problems on pain/discomfort (75\%) and usual activities $(69 \%)$ dimensions. The overall mean FACT-G Total score was 71.8 (15.3). For FACT-G domains, functional well-being, which ranged from $0-28$, was most affected with a score of 17.12 (SD 6.15) followed by emotional well-being at 15.7 (SD 5.2). The mean FKSI-19 total score was 48.8 (SD 13.2). Mean FKSI-DR-symptoms emotional score was low (1.7 out of 4), indicating a strong impact of symptoms on the patients. CTSQ overall score on satisfaction with the therapy was 82.0 (SD 12.9), with a low score of 5.7 (SD 20.9) for treatment expectation, indicating lack of appropriate perceived quality of treatment. For WPAI, 40\% patients were employed and overall work productivity loss was 42.9 (SD 30.0).

Conclusions: There is substantial humanistic burden for patients with aRCC in UK, including diminished health-related quality of life, low satisfaction, and decreased work productivity. New treatment options with increased effectiveness and improved adverse event profiles are warranted to improve these patient-reported outcomes.

PT2977, a Novel HIF-2a Antagonist, Affords Potent Anti-Tumor Activity and Remodels the Immunosuppressive Tumor Microenvironment in Clear Cell Renal Cell Cancer

\author{
Josey, John (Peloton Therapeutics, Dallas, TX, United \\ States)
}

Hypoxia-inducible factor 2a (HIF-2a), a transcription factor, has been established as an oncogenic driver in clear cell renal cell cancer (ccRCC). The first HIF-2a antagonist being evaluated in clinical development, PT2385, has demonstrated clinical activity in ccRCC patients who had previously been treated with multiple lines of therapy. There is continuing effort to characterize additional HIF-2a antagonists possessing attributes that may enhance clinical activity. PT2977 is a novel HIF-2a antagonist with improved potency in preclinical tumor models compared to PT2385. This improvement arises from augmented biochemical and cellular potency, reduced plasma protein binding, and diminished metabolic clearance in vivo relative to PT2385. PT2977 exhibits favorable metabolic stability and pharmacokinetic characteristics when dosed orally 
in multiple preclinical species. Allometric scaling of the preclinical data predicts PT2977 to be suitable for oral once daily dosing in humans. PT2977 inhibits expression of HIF-2a target genes in tumor cells and induces complete stasis or regression in ccRCC xenografts. A strong pharmacokinetics/ pharmacodynamics correlation is observed in tumors from xenograft models treated with PT2977. Gene expression analyses of ccRCC xenografts treated with PT2977 reveal extensive modulation of genes in the tumor cells as well as in tumor associated immune cells. Immune phenotyping of tumors treated with PT2977 confirms that HIF-2a antagonism results in a reduction in the number of immunosuppressive myeloid-derived cells, including neutrophils and macrophages. Treatment with PT2977 also results in an influx of mature dendritic cells. These observations are consistent with aberrant HIF-2a activity exerting an immunosuppressive effect on the tumor microenvironment, in addition to driving angiogenesis and the proliferation and viability of tumor cells. With its favorable preclinical profile, PT2977 is well-positioned to further reveal the broader therapeutic potential of HIF-2a antagonism for the treatment of cancer, as a single agent or in combination with other immune modulating agents.

\section{4}

\section{Quality-adjusted survival of} nivolumab vs. everolimus in patients with previously treated advanced renal cell carcinoma (aRCC): a Q-TWiST analysis

\author{
Shah, Ruchitbhai (Pharmerit International, Bethesda, \\ MD, United States); Botteman, Marc; Solem, Caitlyn; \\ Luo, Linlin (Pharmerit International, Bethesda, MD, \\ United States); Doan, Justin (Bristol Myers Squibb, \\ Wallingford, CT, United States); Cella, David \\ (Northwestern University Feinberg School of Medicine, \\ Chicago, IL, United States); Motzer, Robert (Memorial \\ Sloan Kettering Cancer Center, New York, NY, United \\ States)
}

Background: In the Checkmate 025 trial, nivolumab significantly improved median overall survival (OS; 25.0 vs. 19.6 months [mos]) and the objective response rate (ORR; $25 \%$ vs $5 \%$ ) vs. everolimus in previously treated aRCC patients. The objective of this analysis was to compare the quality adjusted time without symptoms of disease progression or toxicity (Q-TWiST) between nivolumab and everolimus using Checkmate 025 data at a follow up of $\leq 45$ mos.

Methods: OS was partitioned into 3 health states: time without symptoms of disease progression or toxicity (TWiST), time with grade $\geq 3$ adverse event (AE) toxicity after randomization but before progression (TOX), and time after progression (REL). Mean Q-TWiST was calculated by weighting the restricted mean time spent in each health state by a utility of 1.0 for TWiST and 0.5 for TOX and REL. Sensitivity analysis was conducted to compare the Q-TWiST difference between nivolumab and everolimus at regular intervals from 6 mos up to 45 mos. Additionally, a threshold sensitivity analysis assessed the QTWiST difference when utilities of TOX and REL were varied jointly between 0 and 1 . Analyses were performed for pre-specified subgroups (age [ $<65$ vs. $\geq 65$ years old], gender, region [United States, Western Europe, and others], previous antiangiogenic regimens [1 vs. 2], and Memorial Sloan Kettering Cancer Center (MSKCC) prognostic risk group [favorable, intermediate, and poor]). Relative gain in Q-TWiST (i.e., Q-TWIST difference divided by mean OS of everolimus) was calculated. A relative gain $\geq 10 \%$ was considered clinically important.

Results: In the intent-to-treat population, compared to everolimus, nivolumab patients had significantly longer TWiST significantly shorter TOX, longer but non-significant REL, and statistically significant improvements in Q-TWiST of 3.3 mos (relative gain:14.38\%). The relative gain increased from $3.65 \%$ at 6 mos to $14.38 \%$ at 45 mos of follow-up. In threshold sensitivity analyses the Q-TWiST difference (and relative gain) varied from a minimum of $2.4 \operatorname{mos}(10.45 \%)$ when the utilities for $\mathrm{TOX}=1$ and $\mathrm{REL}=0$ to a maximum of $4.2 \operatorname{mos}(18.30 \%)$ when the utilities for TOX $=0$ and REL $=1$. Similar benefits favoring nivolumab were observed when patients were stratified by pre-specified subgroups (Q-TWiST gains ranging from 0.5 to $4.6 \mathrm{mos}$ ).

Conclusions: In Checkmate 025, nivolumab resulted in a statistically significant and clinically important gain in quality adjusted OS vs. everolimus among previously treated aRCC patients. 
Table. Restricted Mean Duration of Key Health States at 45-month follow-up (in mos)

\begin{tabular}{|l|c|c|c|}
\hline & Nivolumab $(\mathrm{N}=410)$ & Everolimus $(\mathrm{N}=411)$ & Difference \\
\hline TOX & $0.1(0.1,0.1)$ & $0.5(0.3,0.6)$ & $-0.4(-0.5,-0.2)$ \\
\hline TWiST & $9.7(8.5,11.0)$ & $7.0(6.2,7.7)$ & $2.7(1.3,4.2)$ \\
\hline REL & $17.0(15.5,18.5)$ & $15.5(14.2,16.9)$ & $1.5(-0.5,3.5)$ \\
\hline Q-TWiST & $18.2(17.0,19.5)$ & $15.0(13.8$ to 15.9$)$ & $3.3(1.7,5)$ \\
\hline
\end{tabular}

\section{5}

\section{Results of lymph node dissection for locally advanced and metastatic renal cell carcinoma}

Blute, Jr., Michael L. (University of Florida, Gainesville, FL, United States); Crispen, Paul L. (University of Florida, Gainesville, FL, United States)

Introduction: Retroperitoneal lymph node dissection (RPLND) at the time of nephrectomy for renal cell carcinoma (RCC) is controversial. Moreover, it is difficult to determine preoperatively those who will benefit from RPLND. The objective of this study is to describe RPLND outcomes at the time of radical nephrectomy in advanced RCC.

Methods: Locally advanced and metastatic RCC patients treated with radical nephrectomy and RPLND from June 2016 to July 2017 were included. Common preoperative clinical and pathological variables were collected to identify associations with high risk disease. Presence of four radiographic variables were assessed preoperatively to determine an RPLND at the time of radical nephrectomy. These included tumor size $>10 \mathrm{~cm}$, lymphadenopathy (LN $>1 \mathrm{~cm}$ diameter in short axis), tumor necrosis (defined as low-enhanced tumor areas), and distant metastases.

Results: Twenty-eight patients who underwent a radical nephrectomy and RPLND were available for study review. There were 17 males and 11 females included with a median age of 61 (IQR 55$68)$. Twenty-two patients (79\%) had clinical stage 3 or higher disease. Median number of lymph nodes removed during RPLND was 13 (IQR 1227). Median number of positive lymph nodes was 3.6 (IQR 1-5.5). Lymph node positive RCC was diagnosed in $6(21 \%)$ patients. Of the patients who had radiographic lymphadenopathy, 6 of $10(60 \%)$ had lymph node positive disease. Metastatic RCC was diagnosed in 8 patients who underwent cytoreductive nephrectomy. Of these patients, 4
(50\%) demonstrated positive lymph nodes following RPLND.

Differences between lymph node positive patients and lymph node negative patients were assessed according to the presence of four radiographic criteria as demonstrated on cross-sectional imaging. Of the 6 lymph node positive patients, all had $\geq 3$ criteria compared to just 2 patients with lymph node negative disease $(\mathrm{p}<0.0001$; Fisher's exact).

Conclusions: In high risk patients with advanced RCC, RPLND may be considered during radical nephrectomy for complete staging and potential enrollment into adjuvant systemic therapy trials. Preoperative assessment of high risk features is important to determine who will ultimately benefit from RPLND.

\section{Rheumatologic adverse events in patients with metastatic renal cell carcinoma treated with immune checkpoint inhibitors}

Ornstein, Moshe C. (Cleveland Clinic Taussig Cancer Institute, Cleveland, $\mathrm{OH}$, United States); Calabrese, Cassandra (Cleveland Clinic Department of Rheumatology, Cleveland, OH, United States); Wood, Laura S. (Cleveland Clinic Taussig Cancer Institute, Cleveland, OH, United States); Kirchner, Elizabeth (Cleveland Clinic Department of Rheumatology, Cleveland, OH, United States); Tyler, Allison; Profusek, Pamela; Allman, Kimberly D.; Martin, Allison (Cleveland Clinic Taussig Cancer Institute, Cleveland, $\mathrm{OH}$, United States); Kontzias, Apostolos (Cleveland Clinic Department of Rheumatology, Cleveland, $\mathrm{OH}$, United States); Grivas, Petros; Garcia, Jorge (Cleveland Clinic Taussig Cancer Institute, Cleveland, $\mathrm{OH}$, United States); Calabrese, Leonard H. (Cleveland Clinic Department of Rheumatology, Cleveland, $\mathrm{OH}$, United States); Rini, Brian I. (Cleveland Clinic Taussig Cancer Institute, Cleveland, $\mathrm{OH}$, United States)

Background: Rheumatologic immune-related adverse events (irAE) in patients treated with check- 
point inhibitors (CPI) are not well characterized. We present the characteristics, treatment, and outcomes of rheumatologic irAEs in CPI-treated metastatic renal cell carcinoma ( $\mathrm{mRCC})$ patients.

Methods: Patients with $\mathrm{mRCC}$ who were treated with CPIs and developed grade $\geq 2$ (per CTCAEv4) rheumatologic irAEs (i.e., arthralgias and myalgias) were retrospectively reviewed. Patient-, disease-, and rheumatologic-related data were collected and summarized as frequency counts and percentages, or medians and ranges.

Results: Nineteen patients were identified. The majority of patients $(68 \%)$ were male; median age at diagnosis was 54 (range, 48-65). All patients had clear cell histology, all had prior nephrectomy, and $53 \%$ were intermediate risk by International Metastatic Renal Cell Carcinoma Database Consortium (IMDC) criteria. Most (58\%) patients received antiangiogenic therapy prior to CPI treatment. CPI therapy included anti-PD-L1 (26\%), anti-PD-1 (42\%), and combined PD-1 / CTLA-4 antibodies (32\%). Median time from CPI initiation to rheumatologic irAE was 5.5 months (range, 0.23 - 51.3). Most $(68 \%)$ patients had 2-4 muscle or joint groups involved. CPI was held in in $17(89 \%)$ patients who developed rheumatologic irAEs. All patients were treated with prednisone. Median initial prednisone dose was $40 \mathrm{mg} / \mathrm{d}$ (range, $10-60 \mathrm{mg} / \mathrm{d}$ ) and median duration of prednisone therapy was 45.3 weeks (range, 2.6-206). Treatment intensification with methotrexate $(16 \%)$, infliximab $(16 \%)$, tocilizumab (11\%), and etanercept (11\%) was required in some patients for rheumatologic symptom control. Of the patients whose CPI was held for rheumatologic irAEs, 24\% restarted CPI therapy following symptoms improvement, $18 \%$ switched to a subsequent mRCC therapy, and 59\% have an ongoing sustained response to therapy (median 10.2 months; range, 0.63 - 46.1) despite no subsequent treatment for mRCC.

Conclusion: Rheumatologic irAEs in CPI-treated mRCC patients vary in timing of presentation, severity of symptoms, and treatment. These patients are best treated in multidisciplinary teams that include a rheumatologist. The prognostic and predictive impact of these irAEs needs to be assessed in a larger patient population.

\section{Safety of Nivolumab in Patients With Clear Cell (CC) or Non-Clear Cell (NCC) Renal Cell Carcinoma (RCC): Results From the Phase IIIb/IV CheckMate 374 Study}

McFarlane, Joshua (Virginia Cancer Institute, Richmond, VA, United States); Olsen, Mark (Tulsa Cancer Institute, Tulsa, OK, United States); Molina, Ana (Weill Cornell Medical College, New York, NY, United States); Bauer, Todd M. (Sarah Cannon Research Institute / Tennessee Oncology, PLLC, Nashville, TN, United States); Tykodi, Scott (University of Washington and Fred Hutchinson Cancer Research Center, Seattle, WA, United States); Somer, Bradley (The West Clinic, Memphis, TN, United States); Fishman, Mayer (Moffitt Cancer Center, Tampa, FL, United States); Reeves, James (Florida Cancer Specialists - South, Fort Myers, FL, United States); Gunuganti, Vijay (Cancer Care Centers of South Texas, San Antonio, TX, United States); Page, Ray (The Center for Cancer and Blood Disorders, Weatherford, TX, United States); Babu, Sunil (Fort Wayne Medical Oncology and Hematology, Fort Wayne, IN, United States); George, Saby (Roswell Park Institute, Buffalo, United States); Van Veldhuizen, Peter (HCA Midwest Division, Kansas City, MO, United States); Zhang, Joshua; Zhao, Huanyu (Bristol-Myers Squibb, Princeton, NJ, United States); Vogelzang, Nicholas (Comprehensive Cancer Centers of Nevada, Las Vegas, NV, United States)

Background: Nivolumab, a fully human IgG4 antibody that blocks programmed death receptor-1 (PD$1)$, is indicated for previously treated advanced RCC. We report the first safety data from the phase IIIb/IV CheckMate 374 study of patients with advanced/metastatic $\mathrm{CC}$ or NCC RCC receiving nivolumab.

Methods: Adults with advanced/metastatic RCC, who received 1-2 prior systemic anti-VEGF treatments for $\mathrm{CC}$ or $0-3$ prior systemic treatments for NCC, and a total of $\leq 3$ prior systemic treatments in the advanced/metastatic setting, were eligible. Patients received $240 \mathrm{mg}$ nivolumab intravenously every 2 weeks for $\leq 24$ months or until confirmed disease progression or unacceptable toxicity. Primary objectives were to evaluate and characterize grade $(\mathrm{G})$ 3-5 immune-mediated AEs (IMAEs; all non-endocrine events requiring immune-modulating medication; any endocrine event with potential immune-related causality). 
Results: 142 patients with CC $(\mathrm{N}=98), \mathrm{NCC}(\mathrm{N}=43)$, or brain metastases $(\mathrm{N}=1)$ received nivolumab. Most CC patients (77\%) had 1 prior systemic therapy for advanced/metastatic disease; most NCC patients $(65 \%)$ were treatment-naïve. Median follow-up was 8.0 months. The types and frequencies of IMAEs were generally consistent between CC and NCC patients. Among the total population, G3-4 IMAE rates were very low and consisted of hepatitis (overall $2.1 \%$; increased ALT, AST, or blood bilirubin, or hyperbilirubinemia $[0.7 \%$ each]) starting within $47-$ 119 days, with all cases resolved within 8-33 days; endocrine events (diabetic ketoacidosis [1.4\%], acute adrenocortical insufficiency [0.7\%]) starting within 46-132 days; and nephritis (0.7\%) starting at day 43 and resolving in 22 days. There were no G3-4 pneumonitis, rash, hyperthyroidism, hypophysitis, or hypersensitivity IMAEs. Rates of treatment-related AEs were similar to/compared favorably with previous nivolumab studies in advanced/metastatic RCC. No G5 events occurred. Efficacy outcomes will be reported when data mature.

Conclusions: A 240-mg flat dose of nivolumab showed acceptable safety, with similarly low rates of grade 3-4 IMAEs in patients with CC or NCC RCC.

\section{8}

\section{Savolitinib versus sunitinib in patients with MET-driven, unresectable and locally advanced or metastatic papillary renal cell carcinoma: SAVOIR, a randomised, phase III trial}

Ghiorghiu, Dana (AstraZeneca, Melbourn, United Kingdom); Jakacki, Regina (AstraZeneca, Gaithersburg, United States); Haddad, Vincent (AstraZeneca, Melbourn, United Kingdom); Kohlmann, Alexander (AstraZeneca, Cambridge, United Kingdom); Frigault, Melanie M (AstraZeneca, Waltham, United States); Ottesen, Lone (AstraZeneca, Cambridge, United Kingdom); Choueiri, Toni K (Dana-Farber Cancer Institute, Boston, United States)

Background: Papillary renal cell carcinoma (PRCC) is the most common of the non-clear cell renal cell carcinomas (RCCs), accounting for $10-15 \%$ of RCCs. However, there are no therapies approved specifically for patients with PRCC, who currently receive treatments approved for clear cell RCC, such as sunitinib. PRCC is often MET-driven (defined as MET kinase domain mutations, MET amplification, chromosome 7 gain and/or HGF amplification). Savolitinib (AZD6094, HMPL-504, volitinib) is a highly selective MET tyrosine kinase inhibitor which demonstrated anti-tumour activity for patients with MET-driven PRCC in a phase II trial.

Trial design: SAVOIR (NCT03091192) is a global, phase III, open-label, randomised, controlled trial evaluating the efficacy and safety of savolitinib, compared with sunitinib, in patients with MET-driven, unresectable, locally advanced or metastatic PRCC. Approximately 180 patients will be randomised at $\sim 50-75$ sites across 5-10 countries. Eligible patients (aged $\geq 18$ with MET-driven PRCC confirmed by a novel, sponsor designated, validated, targeted next generation sequencing assay; a Karnofsky performance status $\geq 80$; and measurable disease at baseline) will be randomised in a 1:1 ratio to receive either continuous savolitinib $600 \mathrm{mg}$ (400 mg if $<50 \mathrm{~kg}$ ) orally, once daily (QD), or sunitinib $50 \mathrm{mg}$ orally QD (4 weeks on/2 weeks off).

The primary objective is to determine the efficacy of savolitinib compared with sunitinib in terms of progression free survival (PFS) as assessed by blinded independent central review [BICR]. Tumour assessments (RECIST 1.1) will be performed at screening and the end of every 6-week cycle until 12 months, and every 12 weeks thereafter until disease progression. Secondary endpoints include overall survival, objective response rate, duration of response, best percentage change in tumour size, disease control rate at 6 and 12 months, safety and tolerability, pharmacokinetics and biomarkers. The impact of savolitinib compared with sunitinib on disease symptoms and quality of life will also be assessed.

\section{9}

\section{Second-Line Treatment of Metastatic Renal Cell Carcinoma: Systematic Review and Network Meta-Analysis}

\author{
Carolina Cauduro ${ }^{1 *}$, Pablo M. Barrios ${ }^{1^{*}}$, Pedro \\ Crivelaro ${ }^{1}$, Luiza Doro ${ }^{1}$, Gabriel Lenz ${ }^{1}$, Márcio \\ Debiasi $^{1,2,3}$, André P. Fay ${ }^{1,2,3}$
${ }^{1}$ PUCRS School of Medicine, Porto Alegre, Brazil
${ }^{2}$ Department of Medical Oncology - Hospital São Lucas da PUCRS, Porto Alegre, Brazil
${ }^{3}$ Latin American Cooperative Oncology Group

*These authors contribute equally for this work 
Introduction: Several agents have been approved for patients with $\mathrm{mRCC}$ who have failed to a firstline VEGF-targeted therapy. No direct comparisons have been performed between those agents. We have performed a systematic review and network metaanalysis to compare and rank the regimens available for second-line treatment in terms of its efficacy and toxicity.

Methods: A systematic search was carried out in MEDLINE, Cochrane Central Register of Controlled Trials and EMBASE. Our primary objective was overall survival (OS). Secondary endpoints include progression free survival (PFS) and toxicity. Inclusion criteria were: phase II or III randomized clinical trials comparing any second-line treatment regimen in patients who had progressed to first-line VEGF-targeted therapy. Biomarkers studies or trials using other immunotherapies rather than immune checkpoint inhibitors were excluded from this analysis. Network meta-analysis [multiple treatment comparison (MTC)] was performed using a Bayesian methodology. MTC estimates use direct and indirect evidence across studies to yield relative comparisons among all included arms for the outcomes of interest. Based on their relative comparisons [relative risks (RR) or hazard ratios (HR)] with their associated credibility intervals (CrI), treatments were ranked, showing the probability of each arm being the best (or the worst) for each outcome.

Results: Literature search retrieved 1410 studies. Of these, only 7 clinical trials met inclusion criteria. Ten treatment arms were identified: axitinib, levatinib, lenvatinib + everolimus (LEV+EVE), everolimus, temsirolimus, sorafenib, nivolumab, cabozantinib, apitolisib and placebo. Temsirolimus and everolimus were arbitrarily considered as a single arm called "mTOR inhibitor". Overall, the network analysis included 3034 patients. HRs are described in table 1. No significant differences in OS were observed across cabozantinib, LEV+EVE and nivolumab arms. However, in terms of PFS nivolumab was found to be inferior when compared to LEV+EVE and cabozantinib. In the ranking for OS and PFS, LEV+EVE had the highest probability of being the most effective second-line treatment (68.56\% and $86.49 \%$, respectively). Nivolumab was ranked as the safest regimen with $100 \%$ probability with a relative risk reductions of $62 \%$ and $56 \%$ in relation to LEV+EVE (RR 0.38; 95\% CrI 0.23-0.55) and cabozantinib (RR 0.44; 95\% CrI 0.32-0.56), respectively.
Conclusions: In this indirect comparison, no significant differences in OS were observed between agents. Nivolumab was the less toxic treatment strategy in this clinical scenario.

40

\section{Systemic therapy for oligo-progressive, metastatic renal cell carcinoma (mRCC) treated with stereotactic radiosurgery (SBRT): to switch or not to switch?}

\author{
Barata, Pedro (Cleveland Clinic Taussig Cancer \\ Institute, Cleveland, $\mathrm{OH}$, United States); Kotesha, \\ Rupesh (Miami Cancer Institute, Miami, FL, United \\ States); Angelov, Lilyana; Chao, Samuel; Mendiratta, \\ Prateek (Taussig Cancer Institute, Cleveland Clinic, \\ Cleveland, OH, United States); Elson, Paul (Quantitative \\ Health Sciences, Cleveland Clinic, Cleveland, $\mathrm{OH}$, \\ United States); Koshkin, Vadim; Ornstein, Moshe; \\ Gilligan, Timothy (Taussig Cancer Institute, Cleveland \\ Clinic, Cleveland, OH, United States); Grivas, Petros \\ (Taussig Cancer Institute, Cleveland Clinic, Cleveland, \\ United States); Wood, Laura; Rini, Brian; Garcia, Jorge \\ (Taussig Cancer Institute, Cleveland Clinic, Cleveland, \\ $\mathrm{OH}$, United States)
}

Introduction: There are limited data regarding the role of changing systemic therapy upon receiving SBRT for oligo-progression (O-PD).

Methods: We reviewed our experience comparing switching $v s$. maintaining systemic therapy in mRCC patients receiving SBRT to brain or osseous metastases for O-PD. Patients who were off systemic therapy for more than 8 weeks before or after SBRT date were excluded.

The treatment response outside SBRT site was evaluated according to RECIST criteria for extraosseous disease and incorporated clinical (symptoms) and radiographic criteria (new lesions in scans) for bone metastases. O-PD included patients who had all progressive lesions treated with SBRT and no other sites of PD outside SBRT site(s).

Based on the timing of systemic therapy switch after SBRT, two groups were identified: (STAY) patients remained on the same systemic treatment; (SWITCH) patients changed systemic therapy after the completion of SBRT. Systemic therapy change or not was made at the treating physician discretion. Treatment duration was defined as the time interval between SBRT date and last day of systemic therapy 
for the STAY group; for the SWITCH group, it was the time interval between C1D1 and last day of the first subsequent therapy after SBRT.

Results: Among $308 \mathrm{mRCC}$ patients who were treated with SBRT to brain or osseous metastases at CCF between 2005-2017, 103 clear-cell mRCC patients met inclusion criteria. Patients who had PD outside SBRT ( $\mathrm{n}=33$ ) or with no response information available $(n=3)$ were excluded. Final analysis included 67 patients with median age 60 (range, 3975), 78\% male, 88\% ECOG 0-1, with 55\% IMDCintermediate risk.

Thirty-five patients had SBRT to brain (frontal $51 \%$, parietal $37 \%$, cerebellum $35 \%$; median number lesions=2) and 32 patients had SBRT to bone (spine $88 \%$, median number lesions $=1$ ). Local control after SBRT was achieved in $85 \%$ of treated sites.

At the time of SBRT, most patients were being treated with sunitinib (40\%), axitinib (24\%) or everolimus (10\%). Systemic treatment was first-line for $39 \%$ of patients, second-line for $31 \%$ and thirdline or greater for 30\%. Best RECIST response was $\mathrm{CR}$ in $3 \%$ and $\mathrm{PR}$ in $33 \%$.

Patients included in the STAY $(n=47)$ and SWITCH $(n=20)$ groups had generally similar characteristics and were treated with a median number of 1 and 2 subsequent lines of treatment after SBRT, respectively. The median time on therapy for STAY and SWITCH groups was 5.2 months $(95 \%$ CI $4.4-$ $6.0)$ and 5.0 months (95\% CI 4.1-5.9), respectively $(\mathrm{p}=0.659)$. The median OS for the STAY group was 19.1 months (95\% CI 7.1-31.4), compared with 27.1 months (95\% CI 12.1-42.1) for the SWITCH group $(\mathrm{p}=0.575)$.

Conclusions: Our findings suggest that SBRT for patients with mRCC and O-PD in brain or bones is feasible and provides excellent local control. Maintaining patients on their current systemic therapy in the setting of O-PD does not compromise treatment durability, progression or survival.

\section{1}

\section{The Association between Insurance Status and Survival in Metastatic Renal Cell Carcinoma in the United States}

Chen, Yu-Wei, Tullio, Kate; Ornstein, Moshe; Grivas, Petros; Garcia, Jorge; Rini, Brian (Taussig Cancer Center, Cleveland Clinic, Cleveland, United States)

Background: About $50 \%$ of patients diagnosed with metastatic renal cell carcinoma (mRCC) are aged $<65$, meaning most of them are not Medicareeligible and they mainly rely on private insurance for healthcare. Prior studies have reported treatment cost increases in the era of targeted therapy for $\mathrm{mRCC}$ and this may impede access to cancer care. Our study aimed to investigate the impact of insurance status on receipt of treatment and survival in patients diagnosed with $\mathrm{mRCC}$.

Methods: National Cancer Database was used to identify patients diagnosed with $\mathrm{mRCC}$ in the targeted therapy era in 2006-2013. Multivariable logistic regression was used to investigate the association between insurance status and receipt of targeted therapy or cytoreductive nephrectomy. Multivariable Cox regression analysis was used to assess the association between insurance status and mortality. Propensity score-based analysis was used to adjust Kaplan-Meier survival curve. Covariates adjusted in our study including tumor characteristics and patient sociodemographic factors.

Results: There were 23,808 patients included in our study population: 12,189 (51\%) were aged $<65$ and $11,619(49 \%)$ were aged $\geq 65$. The primary payor for patients aged $<65$ was private insurance $(63 \%)$, followed by Medicaid (14\%) and Medicare (11\%) while $11 \%$ were uninsured. The primary payor for patients aged $\geq 65$ was Medicare $(83 \%)$, followed by private insurance (13\%) and Medicaid (1.9\%) and $0.9 \%$ were uninsured. In multivariable regression analysis: compared with patients with private insurance status, patients who were uninsured (adjusted odds ratio (AOR): 0.62, 95\% CI: 0.55-0.70, p-value: $<.0001$ ), with Medicaid (AOR: 0.81, 95\% CI: 0.740.90, p-value: $<0.001$ ) or with Medicare (AOR: 0.79, 95\% CI: 0.73-0.86, p-value: $<0.001$ ) were less likely to receive targeted therapy; patients who were uninsured, with Medicaid, or with Medicare were less likely to receive cytoreductive nephrectomy. 
In multivariable Cox regression analysis: compared with private insurance status, patients who were uninsured (adjusted hazard ratios (AHR): 1.22, $95 \%$ CI: 1.13-1.30, p-value: < .0001), with Medicaid (AHR: 1.23, 95\% CI: 1.16-1.31, p-value: <.0001), or with Medicare (AHR: 1.19, 95\% CI: 1.14-1.25, p-value: $<.0001)$ were associated with higher mortality risk. There was interaction between insurance status and age $(\mathrm{P}$-value $=0.0004)$. In patients aged $<65$, propensity-score adjusted 12-month survival was higher in patients with private insurance $(48 \%$ vs $39 \%$, AHR: 1.25 , p-value $<.0001)$; in patients aged $\geq 65$, patients with private insurance status had higher 12 -month survival ( $35 \%$ vs $31 \%$, AHR: 1.03 , p-value: 0.005).

Conclusions: Our results revealed that patients with private insurance status were more likely to receive treatment for mRCC and had better survival in the targeted therapy era. This survival disparity was more significant in patients aged $<65$, suggesting insurance status determined access to mRCC treatment while the difference was mitigated by Medicare in patients aged $\geq 65$.

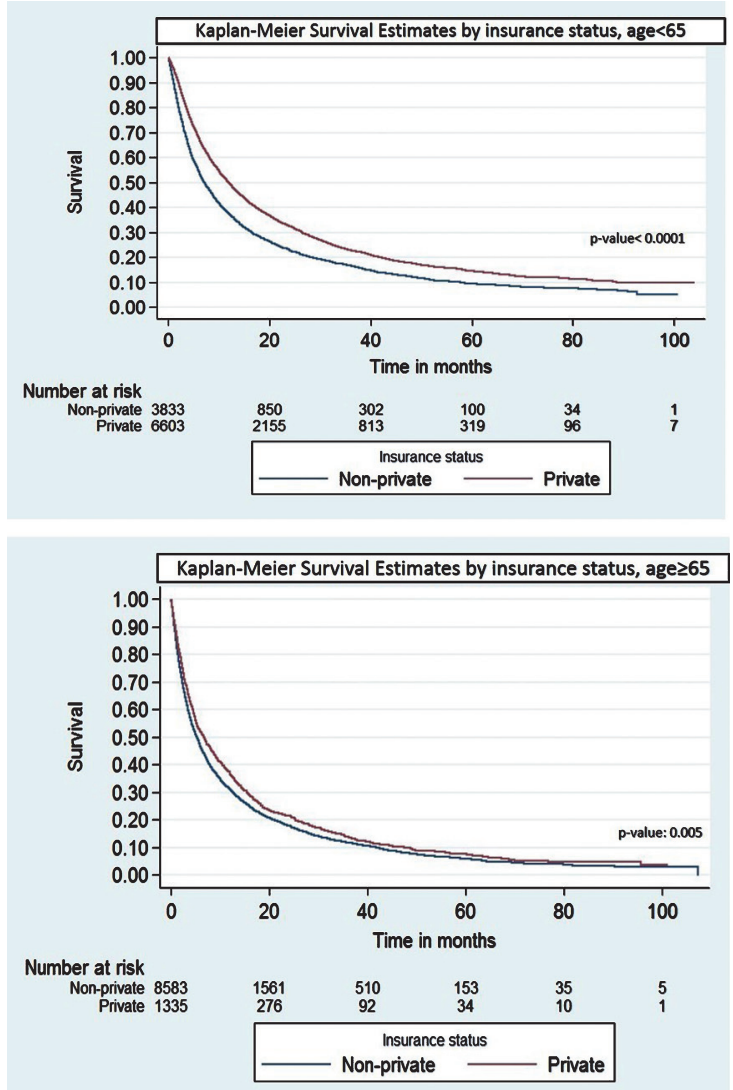

Table: Logistic regression for receipt of targeted therapy

\begin{tabular}{|c|c|c|}
\hline & AOR (95\% CI) & P-value \\
\hline \multicolumn{3}{|l|}{ Insurance Status } \\
\hline Private & Ref & \\
\hline None & $0.62(0.55-0.70)$ & $<.0001$ \\
\hline Medicaid & $0.81(0.74-0.90)$ & $<.0001$ \\
\hline Medicare & $0.79(0.73-0.86)$ & $<.0001$ \\
\hline Other government & $0.59(0.47-0.74)$ & $<.0001$ \\
\hline \multicolumn{3}{|l|}{ Age } \\
\hline$<65$ & Ref & \\
\hline$\geq 65$ & $0.66(0.61-0.72)$ & $<.0001$ \\
\hline \multicolumn{3}{|l|}{ Sex } \\
\hline Female & Ref & $<.0001$ \\
\hline Male & $1.19(1.13-1.26)$ & \\
\hline \multicolumn{3}{|l|}{ Race } \\
\hline Non-Hispanic White & Ref & \\
\hline Hispanic White & $0.90(0.81-1.01)$ & 0.07 \\
\hline Black & $0.96(0.87-1.05)$ & 0.35 \\
\hline Other & $1.02(0.88-1.19)$ & 0.78 \\
\hline \multicolumn{3}{|l|}{ Charlson comorbidity score } \\
\hline $\begin{array}{l} \\
\end{array}$ & Ref & \\
\hline 1 & $0.92(0.86-0.98)$ & 0.012 \\
\hline 2 & $0.67(0.61-0.74)$ & $<.0001$ \\
\hline
\end{tabular}


Table: (Continued)

\begin{tabular}{|c|c|c|}
\hline AJCC T stage & & \\
\hline $\mathrm{T} 1$ & Ref & \\
\hline $\mathrm{T} 2$ & $1.33(1.22-1.44)$ & $<.0001$ \\
\hline T3 & $1.20(1.11-1.30)$ & $<.0001$ \\
\hline $\mathrm{T} 4$ & $1.19(1.07-1.32)$ & 0.001 \\
\hline \multicolumn{3}{|l|}{ AJCC N stage } \\
\hline N0 & Ref & \\
\hline N1 & $1.22(1.14-1.30)$ & $<.0001$ \\
\hline \multicolumn{3}{|l|}{ Facility type } \\
\hline Non-academic & Ref & \\
\hline Academic & $0.96(0.91-1.01)$ & 0.122 \\
\hline \multicolumn{3}{|l|}{ Education level $<$ high school level } \\
\hline$>=21 \%$ & Ref & \\
\hline $13 \%-20.9 \%$ & $1.12(1.03-1.21)$ & 0.009 \\
\hline $7-12.9 \%$ & $1.15(1.05-1.26)$ & 0.003 \\
\hline$<7 \%$ & $1.24(1.11-1.39)$ & 0.0001 \\
\hline \multicolumn{3}{|l|}{ Household income (\%) } \\
\hline$<\$ 38,000$ & Ref & \\
\hline$\$ 38,000-47,999$ & $1.06(0.97-1.15)$ & 0.19 \\
\hline$\$ 48,000-62,999$ & $1.06(0.96-1.16)$ & 0.25 \\
\hline$>=63,000$ & $1.02(0.92-1.14)$ & 0.71 \\
\hline \multicolumn{3}{|l|}{ Residence } \\
\hline Metropolitan & Ref & \\
\hline Urban & $1.10(1.02-1.18)$ & 0.014 \\
\hline Rural & $1.24(1.04-1.46)$ & 0.015 \\
\hline \multicolumn{3}{|l|}{ Year of diagnosis } \\
\hline $2006-2007$ & Ref & \\
\hline $2008-2010$ & $1.20(1.12-1.29)$ & $<.0001$ \\
\hline $2011-2013$ & $1.32(1.23-1.42)$ & $<.0001$ \\
\hline
\end{tabular}

Table: Logistic regression for receipt of cytoreductive nephrectomy

\begin{tabular}{|c|c|c|}
\hline & AOR $(95 \%$ CI) & P-value \\
\hline \multicolumn{3}{|l|}{ Insurance Status } \\
\hline Private & Ref & \\
\hline None & $0.54(0.47-0.62)$ & $<.0001$ \\
\hline Medicaid & $0.61(0.54-0.69)$ & $<.0001$ \\
\hline Medicare & $0.77(0.70-0.84)$ & $<.0001$ \\
\hline Other Government & $0.43(0.33-0.57)$ & $<.0001$ \\
\hline \multicolumn{3}{|l|}{ Age } \\
\hline$<65$ & Ref & \\
\hline$\geq 65$ & $0.56(0.51-0.61)$ & $<.0001$ \\
\hline \multicolumn{3}{|l|}{ Sex } \\
\hline Female & Ref & \\
\hline Male & $1.10(1.03-1.17)$ & 0.007 \\
\hline \multicolumn{3}{|l|}{ Race } \\
\hline Non-Hispanic White & Ref & \\
\hline Hispanic White & $1.04(0.92-1.18)$ & 0.54 \\
\hline Black & $0.72(0.64-0.80)$ & $<.0001$ \\
\hline Other & $0.99(0.84-1.18)$ & 0.98 \\
\hline \multicolumn{3}{|l|}{ Charlson comorbidity score } \\
\hline 0 & Ref & \\
\hline 1 & $1.15(1.07-1.24)$ & 0.002 \\
\hline
\end{tabular}


Table: (Continued)

\begin{tabular}{|c|c|c|}
\hline 2 & $0.71(0.63-0.80)$ & $<.0001$ \\
\hline \multicolumn{3}{|l|}{ AJCC T stage } \\
\hline $\mathrm{T} 1$ & Ref & \\
\hline $\mathrm{T} 2$ & $1.95(1.77-2.14)$ & $<.0001$ \\
\hline $\mathrm{T} 3$ & $2.51(2.29-2.75)$ & $<.0001$ \\
\hline T4 & $0.98(0.86-1.11)$ & 0.77 \\
\hline \multicolumn{3}{|l|}{ AJCC N stage } \\
\hline N0 & Ref & \\
\hline N1 & $0.55(0.51-0.59)$ & $<.0001$ \\
\hline \multicolumn{3}{|l|}{ Facility type } \\
\hline Non-academic & Ref & \\
\hline Academic & $1.70(1.59-1.81)$ & $<.0001$ \\
\hline \multicolumn{3}{|l|}{ Education level } \\
\hline$>=21 \%$ & Ref & \\
\hline $13 \%-20.9 \%$ & $1.07(0.97-1.18)$ & 0.21 \\
\hline $7-12.9 \%$ & $1.09(0.98-1.21)$ & 0.12 \\
\hline$<7 \%$ & $1.17(1.03-1.34)$ & 0.02 \\
\hline \multicolumn{3}{|l|}{ Household income (\%) } \\
\hline$<\$ 38,000$ & Ref & \\
\hline$\$ 38,000-47,999$ & $1.09(0.98-1.20)$ & 0.106 \\
\hline$\$ 48,000-62,999$ & $1.13(1.02-1.27)$ & 0.026 \\
\hline$>=63,000$ & $1.22(1.08-1.39)$ & 0.002 \\
\hline \multicolumn{3}{|l|}{ Residence } \\
\hline Metropolitan & Ref & \\
\hline Urban & $1.14(1.04-1.24)$ & 0.027 \\
\hline Rural & $1.29(1.06-1.57)$ & 0.027 \\
\hline \multicolumn{3}{|l|}{ Year of diagnosis } \\
\hline $2006-2007$ & Ref & \\
\hline $2008-2010$ & $1.24(1.13-1.35)$ & $<.0001$ \\
\hline 2011-2013 & $1.21(1.10-1.32)$ & $<.0001$ \\
\hline
\end{tabular}

Table: Cox regression hazard model

\begin{tabular}{|c|c|c|}
\hline & AHR (95\% CI) & P-value \\
\hline Insurance Status & & \\
\hline Private & Ref & \\
\hline None & $1.22(1.13-1.30)$ & $<.0001$ \\
\hline Medicaid & $1.23(1.16-1.31)$ & $<.0001$ \\
\hline Medicare & $1.19(1.14-1.25)$ & $<.0001$ \\
\hline Other government & $1.20(1.06-1.36)$ & 0.005 \\
\hline Age & & $<.0001$ \\
\hline S65 & $1.25(1.19-1.30)$ & \\
\hline Sex & & 0.0003 \\
\hline Female & Ref & \\
\hline Male & $0.94(0.91-0.97)$ & \\
\hline & & $<.0001$ \\
\hline Non-Hispanic White & $0.83(0.78-0.89)$ & 0.02 \\
\hline Hispanic White & $1.07(1.01-1.13)$ & 0.97 \\
\hline Black & $0.99(0.91-1.09)$ & \\
\hline Other & & \\
\hline
\end{tabular}


Table: (Continued)

\begin{tabular}{|c|c|c|}
\hline Charlson comorbidity score & & \\
\hline 0 & Ref & \\
\hline 1 & $1.15(1.10-1.19)$ & $<.0001$ \\
\hline 2 & $1.39(1.32-1.46)$ & $<.0001$ \\
\hline \multicolumn{3}{|l|}{ AJCC T stage } \\
\hline $\mathrm{T} 1$ & Ref & \\
\hline $\mathrm{T} 2$ & $1.04(0.99-1.09)$ & 0.12 \\
\hline T3 & $1.08(1.03-1.14)$ & 0.001 \\
\hline $\mathrm{T} 4$ & $1.33(1.26-1.42)$ & $<.0001$ \\
\hline \multicolumn{3}{|l|}{ AJCC N stage } \\
\hline N0 & Ref & \\
\hline N1 & $1.51(1.44-1.59)$ & $<.0001$ \\
\hline \multicolumn{3}{|l|}{ Facility type } \\
\hline Non-academic & Ref & $<.0001$ \\
\hline Academic & $0.84(0.82-0.87)$ & \\
\hline \multicolumn{3}{|l|}{ Histology Type } \\
\hline Clear Cell & Ref & \\
\hline Papillary & $0.90(0.83-0.99)$ & 0.027 \\
\hline Chromophobe & $0.70(0.56-0.87)$ & 0.001 \\
\hline Collecting duct & $1.50(1.22-1.83)$ & $<.0001$ \\
\hline Sarcomatoid & $1.62(1.51-1.73)$ & $<.0001$ \\
\hline $\begin{array}{r}\text { Other } \\
\end{array}$ & $1.40(1.33-1.47)$ & $<.0001$ \\
\hline \multicolumn{3}{|l|}{ Fuhrman grade } \\
\hline 1 & Ref & \\
\hline 2 & $0.86(0.76-0.98)$ & 0.027 \\
\hline 3 & $1.18(1.04-1.33)$ & 0.008 \\
\hline 4 & $1.23(1.08-1.40)$ & 0.001 \\
\hline \multicolumn{3}{|l|}{ Education level } \\
\hline$>=21 \%$ & Ref & \\
\hline $13 \%-20.9 \%$ & $0.99(0.94-1.04)$ & 0.70 \\
\hline $7-12.9 \%$ & $0.98(0.92-1.04)$ & 0.52 \\
\hline$<7 \%$ & $0.98(0.92-1.05)$ & 0.59 \\
\hline \multicolumn{3}{|l|}{ Household income (\%) } \\
\hline$<\$ 38,000$ & Ref & \\
\hline$\$ 38,000-47,999$ & $1.03(0.98-1.08)$ & 0.21 \\
\hline$\$ 48,000-62,999$ & $0.96(0.91-1.02)$ & 0.18 \\
\hline$>=63,000$ & $0.90(0.85-0.96)$ & 0.002 \\
\hline \multicolumn{3}{|l|}{ Residence } \\
\hline Metropolitan & Ref & \\
\hline Urban & $0.99(0.96-1.04)$ & 0.89 \\
\hline Rural & $0.92(0.83-1.01)$ & 0.09 \\
\hline \multicolumn{3}{|l|}{ Year of diagnosis } \\
\hline $2006-2007$ & Ref & \\
\hline $2008-2010$ & $0.97(0.93-1.00)$ & 006 \\
\hline $2011-2013$ & $0.81(0.78-0.85)$ & $<.0001$ \\
\hline
\end{tabular}




\section{2}

\section{The association of sarcopenia and tumor aggressiveness in clear cell renal cell carcinoma}

Sanchez, Alejandro (Memorial Sloan-Kettering, New York, NY, United States); Petruzella, Stacey (Memorial Sloan Kettering Cancer Center, New York, NY, United States); Samson, Marguerite; Akin, Oguz (Memorial Sloan Kettering Cancer Center, New York, NY, United States); Paris, Mike (University of Waterloo, Waterloo, ON, Canada); Mourtzakis, Marina (Department of Kinesiology, University of Waterloo, Waterloo, ON, Canada); Hakimi, Ari; Russo, Paul (Department of Urology, Memorial Sloank Kettering Cancer Center, New York, NY, United States); Helena, Furberg (Department of Epidemiology \& Biostatistics, Memorial Sloan Kettering Cancer Center, New York, NY, United States)

Introduction: Sarcopenia is associated with poor prognosis, while obesity is paradoxically associated with improved outcomes among renal cell carcinoma (RCC) patients. Underlying differences in tumor biology related to combinations of sarcopenia and obesity have not been described.

Methods: We examined how sarcopenia was associated with molecular subtypes of clear cell RCC (ccRCC) among patients who were obese or nonobese. The cohort consisted of 48 ccRCC patients treated by nephrectomy at our institution who were transcriptomically-profiled by the Cancer Genome Atlas. Computerized tomography scans without contrast performed within 60-days of surgery were reviewed to determine skeletal muscle index (SMI). Sarcopenia (yes/no) was classified according to gender-specific international consensus definitions (SMI of $<55 \mathrm{~cm}^{2} / \mathrm{m}^{2}$ for men and $<39 \mathrm{~cm}^{2} / \mathrm{m}^{2}$ for women). Obesity (yes/no) was defined as pre-surgical body mass index $\geq 30 \mathrm{~kg} / \mathrm{m}^{2}$. The ClearCode 34 gene expression classifier categorized patients into either ccA (less aggressive) or ccB (more aggressive) molecular subtypes. Chi-square or Fisher's exact tests describe associations between sarcopenia and molecular subtype by obesity status. Statistical significance was regarded as a $\mathrm{p}$-value of $<0.05$.

Results: The cohort was predominantly male (79\%), white $(97 \%)$, and had localized disease $(67 \%)$. Median age was 57 years (IQR: $34-79)$. Overall, $46 \%$ of patients were obese, $46 \%$ were sarcopenic, and $42 \%$ of tumors were $\mathrm{ccB}$ subtype. Among patients who were not obese, aggressive ccB subtype was more common in sarcopenic $(71 \%)$ than non-sarcopenic patients (34\%). A similar pattern was observed among patients who were obese; aggressive $\mathrm{ccB}$ subtype was more common in sarcopenic (40\%) than non-sarcopenic patients (18\%).

Conclusions: While preliminary, our findings suggest that sarcopenia is associated with aggressive ccRCC regardless of obesity and lend biologic support to the observation that sarcopenia is associated with poor prognosis. It is not clear whether sarcopenia is a cause or consequence of tumor aggressiveness. Pathway analyses to explore specific mechanisms underlying these observations are underway.

Funding: Chanel grant (HF) and Ruth L. Kirschstein Research Service Award T32CA082088 (AS).

43

\section{The impact of bone metastasis location in the clinical outcome of patients with metastatic renal cell carcinoma (mRCC): an analysis from the Latin American Renal Cancer Group (LARCG)}

Abreu, Diego (Pasteur Hospital, Montevideo, Uruguay); Gueglio, Guillermo; Jurado, Alberto (Italiano Hospital, Buenos Aires, Argentina); Meza, Luiz (Instituto Nacional de Enfermedades Neoplásicas, LI, Peru); Scorticati; Lopez, Maximiliano (Hospital de Clinicas, BAP, Argentina); de Cassio Zequi, Stenio; Henriques da Costa, Walter (A.C. Camargo Cancer Center, SP, Brazil); Yandian, Juan (Hospital de Clínicas, Uruguay); Ubillos, LUis (Hospital de Clinicas, MO, Uruguay); Ameri, Carlos A. (Hospital Aleman, Buenos Aires, Argentina); Nolazco, Alejeandro; Martinez, Pablo (Hospital de Clinicas, BAP, Argentina); Carvalhal, Gustavo F. (PUCRS School of Medicine, Porto Alegre, Brazil); Cauduro, Carolina; Barrios, Pablo (PUCRS School of Medicine, Porto Alegre, RS, Brazil); Bengio, Ruben; Arribillaga, Leandro (Clinica Profesor Bengio, Cordoba, COR, Argentina); Langenhin, Raúl; Muguruza, Diego (COMEPA, Paysandu, PA, Uruguay); Gadu, José; Bravo, Edgar (Hospital Militar, Ciudad de México, Mexico); Castillejos, Ricardo; Rodríguez-Covarrubias, Francisco (Instituto Nacional de Ciencias Médicas y Nutrición Salvador Zubirán (INCNSZ), Ciudad de México, Mexico); Mingote, Pablo (Policlinico Neuquén, Neuquén, Argentina); Ginastar, Nicolás (Policlinico Neuquén, Neuquén, United States); Puente, Roberto (Hospital de Clinicas, Montevideo, MO, Uruguay); Decia, Roberto (Hospital Pasteur, Montevideo, MO, Uruguay); Cardoso 
Guimarães, Gustavo (A.C. Camargo Cancer Center, Sao Paulo, SP, Brazil); Palau, Joan (Fundación Puigvert, Barcelona, BA, Spain); Fay, André P. (PUCRS School of Medicine Instituto do Câncer Hospital Mãe de Deus, Porto Alegre, Brazil)

Background: Tumor burden and site of metastatic disease are well-established prognostic factors in many malignancies, including $\mathrm{mRCC}$. This analysis aims to evaluate the impact of bone metastasis location in the clinical outcome of $\mathrm{mRCC}$ patients.

Methods: A retrospective analysis using the collaborative cohort of mRCC patients enrolled in the database from LARCG was performed. This consortium includes 45 centers from 9 countries including Uruguay, Brasil, Argentina, Mexico, Peru, Chile, Bolivia, and Spain. Clinico-pathological characteristics, 24-months-survival, overall survival (OS), as well as sites of bone metastatsis were collected. Clinico-pathological characteristics are presented descriptively. Cox regression was utilized to estimate the association of bone metastasis location and clinical outcomes.

Results: From 1990 to 2015, 4060 patients were included in the LARCG dataset. The majority of patients had localized RCC. 530 (14.5\%) patients had metastasis at diagnosis. Out of 530, 56 had exclusively bone metastasis. Median follow-up was 20.8 months (0-188). Survival data was available for 55 out of those 56 patients. Thirty-six (64.3\%) patients were male. The median age of was 59.5 years (40$85)$, and $48(90.6 \%)$ had symptoms at presentation. Primary nephrectomy was performed in 46 patients $(82.1 \%)$ and systemic therapy was VEGF-targeted therapy and cytokines in 35 and 1 patients, respectively. Non-vertebral bone metastasis (NVBM) were identified in 33(58.9\%) patients and vertebral metastasis (VBM) in 23(41.1\%) patients. Median OS for the overall cohort of mRCC patients was 24 months. The 24-months OS was, $87 \%$ for patients with NVBM vs. $50 \%$ for VBM patients (HR: $2.70,95 \%$ CI: $1.10-6.61 ; p=0.029$ - univariate analysis). Sarcomatoid component was associated with vertebral metastatsis $(\mathrm{p}=0.041)$.

Conclusions: Although we have analyzed a small number of patients, vertebral bone metastasis appear to be associated with shorter 24-months OS, suggesting that location of bone metastasis may impact the clinical outcome of patients with mRCC.
44

\section{Three-Year Efficacy and Safety Update From the Phase III CheckMate 025 Study of Nivolumab Versus Everolimus in Patients With Advanced Renal Cell Carcinoma (aRCC)}

Sharma, Padmanee (University of Texas, MD Anderson Cancer Center, Houston, TX, United States); Tykodi, Scott (University of Washington and Fred Hutchinson Cancer Research Center, Seattle, United States); Escudier, Bernard (Institut Gustave Roussy, Villejuif, France); Carducci, Michael (Johns Hopkins Medicine The Sidney Kimmel Comprehensive Cancer Center, Baltimore, United States); Oudard, Stephane (Hôpital Européen Georges Pompidou, Paris, United States); Hammers, Hans J (Johns Hopkins Sidney Kimmel Comprehensive Cancer Center, Baltimore, United States); George, Saby (Roswell Park Cancer Institute, Buffalo, United States); Castellano, Daniel (Hospital Universitario 12 De Octubre, Madrid, Spain); Alva, Ajjai S. (University of Michigan, Ann Arbor, United States); Richardet, Martin Eduardo (Fundacion Richardet Longo - Instituto Oncologico de Cordoba, Cordoba, Argentina); Chevreau, Christine (IUCT-O Institut Claudius Regaud, Toulouse, France); Plimack, Elizabeth R (Fox Chase Cancer Center, Philadelphia, United States); Srinivas, Sandhya (Stanford Cancer Institute, Stanford, United States); Procopio, Giuseppe (Fondazione Istituto Nazionale Tumori, Milano, United States); Sosman, Jeffrey A. (Vanderbilt University Medical Center, Nashville, United States); McDermott, David F. (Beth Israel Deaconess Medical Center, Dana-Farber/Harvard Cancer Center, Boston, United States); Choueiri, Toni K. (Dana-Farber Cancer Institute/Brigham and Women's Hospital, Boston, United States); Berghorn, Elmer J; Yang, Lingfeng (Bristol-Myers Squibb, Princeton, United States); Motzer, Robert J. (Memorial Sloan Kettering Cancer Center, New York, United States)

Background: CheckMate 025 ( $\geq$ 14-month followup) demonstrated superior overall survival (OS) with nivolumab versus everolimus (25.0 vs 19.6 months) and a higher objective response rate (ORR; $25 \%$ vs $5 \%$ ) in previously treated patients with aRCC (NEJM 2015). Here we report an exploratory analysis of 3-yr efficacy and safety.

Methods: Adults with clear-cell aRCC that progressed after 1-2 antiangiogenic therapies were randomized (1:1) to nivolumab $3 \mathrm{mg} / \mathrm{kg}$ IV every 2 weeks or everolimus $10 \mathrm{mg}$ orally once daily until progression or unacceptable toxicity. Endpoints: 
primary, OS; key secondary: ORR, progression-free survival (PFS), adverse events (AEs).

Results: Overall, 410 patients and 411 patients were randomized to nivolumab and everolimus, respectively (27 and 3 continue to receive treatment as randomized). With median follow-up of 24 months with nivolumab and 19 months with everolimus, median OS was 25.8 months and 19.7 months, respectively (hazard ratio [HR], 0.74; $P=0.0005$ ), with 2-year OS rates of $52 \%$ and $42 \%$ and 3 -year OS rates of $39 \%$ and 30\%. Investigator-assessed unconfirmed ORR was consistent with the primary analysis: $26 \%$ (nivolumab) and 5\% (everolimus). Median duration of response was 12.3 months for nivolumab responders and 12.0 months for everolimus responders. Ongoing response was noted in 18\% (17/93) of nivolumab responders and $6 \%(1 / 17)$ of everolimus responders. Median (95\% confidence interval) PFS was 4.2 months (3.7-5.4) with nivolumab and 4.5 months (3.8-5.5) with everolimus (HR, 0.85; $P=0.0371$ ). Incidence and type of treatment-related AEs were consistent with the primary analysis and remained lower with nivolumab $(80 \%$; grade $3-4$, $21 \%$ ) versus everolimus (89\%; grade 3-4, 37\%). Most treatment-related select AEs with nivolumab resolved (75\%-100\%, by AE category), except for endocrine AEs (38\%), which required permanent hormone replacement. Quality-of-life improvement with nivolumab was consistent with earlier analyses.

Conclusions: In this 3-year update, nivolumab continues to demonstrate durable responses and a survival benefit versus everolimus in previously treated patients with aRCC. The safety profile is favorable and consistent with the primary analysis, and most AEs were manageable with the majority having resolved.

\section{5}

\section{TiNiVo: A Phase Ib Dose Escalation Trial of Tivozanib and Nivolumab in Renal Cell Carcinoma}

Albiges, Laurence (Gustave Roussy, Villejuif, France); Escudier, Bernard (Gustave Roussy, Villejuif, France); Needle, Michael (Aveo Oncology, Cambridge, MA, United States); Barthelemy, Philippe (Hopitaux universitaires de strasbourg, Strasbourg, France)

Background: Combinations of vascular endothelial growth factor receptor tyrosine kinase inhibitiors
(VEGFR-TKIs) and checkpoint inhibitors have proven to be both active and toxic. Tivozanib is a VEGFR-TKI with high specificity and lower incidence of class effect adverse events. In this phase I study we combined tivozanib with nivolumab in patients with metastatic renal cell carcinoma.

Methods: Using a standard $3+3$ design we determined the safety, tolerability, and maximum tolerated dose of tivozanib in combination with nivolumab in patients with metastatic renal cell carcinoma. Tivozanib was administered orally at two dose levels, $1.0 \mathrm{mg}$ and $1.5 \mathrm{mg}$, once daily for 21 days every 28 day cycle in combination with nivolumab $240 \mathrm{mg}$ every 14 days intravenously.

Results: Six patients were treated. Three were untreated and 3 patients had previously progressed following treatment with sunitinib. The median age was 59; 4 patients were ECOG 0 and 2 ECOG 1; and there were 4 males. No patient had a dose limiting toxicity in cycle 1 . The most common adverse events were asthenia seen in 3 patients and diarrhea, stomatitis, arthralgia, and dysphonia, all seen in 2 patients. Hypertension, elevations of liver enzymes, and hand foot syndrome, were seen in one patient each. In cycle 1 no adverse event was higher than grade 2, although one event of grade 3 stomatitis was observed in a later cycle. No immune related adverse events were seen. There was no discernible difference between the two dose cohorts. All patients are ongoing and have started cycle 3 . No patient progressed at the end of cycle 2 .

Conclusions: As expected from a VEGFR-TKI with high specificity and a preferable toxicity pattern as a single agent, the combination of tivozanib with nivolumab has an adverse event pattern that appears preferable to combinations using other, less specific, VEGR-TKIs. The MTD was not identified. This data is based on limited exposure and should be interpreted with caution. More follow up is forthcoming from these patients, as well as data from an expansion cohort of patients treated at full dose of both agents, $1.5 \mathrm{mg}$ of tivozanib with $240 \mathrm{mg}$ of nivolumab. This dose would be the dose recommended for further studies. 
46

\section{Treatment of metastatic renal cell carcinoma with the mushroom toxin orellanine}

\author{
Sven Lundstam ${ }^{1}$, Börje Haraldsson ${ }^{2}$, Ulrika Stierner \\ and Jenny Nyström² \\ ${ }^{1}$ Department of Urology, Sahlgrenska University \\ Hospital, Gothenburg, Sweden, ${ }^{2}$ Institute of \\ Neuroscience and Physiology, Sahlgrenska Academy, \\ University of Gothenburg, Gothenburg, Sweden \\ ${ }^{3}$ Department of Oncology, Sahlgrenska University \\ Hospital, Gothenburg, Sweden
}

Poisoning by the mushroom "deadly webcap" (Cortinarius sp.) causes irreversible kidney damage but there are no known effects on other organs. The mushroom toxin, orellanine, selectively targets proximal tubular cells both in rats and in humans by disturbing the cell metabolism causing decreased protein synthesis and apoptosis. Clear cell and papillary renal cell carcinoma originate from proximal tubule cells. We have therefore studied if orellanine also has an effect on renal carcinoma cells

Material and methods: A method for peritoneal dialysis in the rat has been developed. Renal cell carcinoma cell lines have been incubated with and without orellanine. Renal cell carcinoma has been transplanted to rats and orellanine treated animals have been compared to control animals. Primary cultures of fresh tumor tissue from patients operated for renal cell carcinoma (clear cell and papillary) has also been incubated with and without orellanine.

Results: Orellanine induced a pronounced and concentration-dependent decline in viability in renal cancer cell lines from primary tumors and from metastatic lesions but not in control cells (umbilicus epithelium, hepatocytes, carcinoma of the breast). Orellanine induced apoptosis and tumor shrinkage in renal cell carcinoma transplanted to rats. Orellanine treated rats had $400 \%$ more necrosis in the tumor compared to controls. Orellanine induced a dose dependent decrease in viability of human kidney cancer cells (both primary tumors and mets) in vitro. Based on these findings a drug development programme has been launched based on chemically synthesized orellanine.

A phase 1 study is planned to study safety and effects of orellanine on patients in dialysis with metastatic renal cell carcinoma with resistance to other oncological treatment. The study will be launched early 2018 at Sahlgrenska University Hospital. Initially we will include Scandinavian patients only but since the patient group is limited, the aim is then to expand the study by including patients from other countries.

Conclusion: Orellanine is a potential treatment for renal cell carcinoma with a unique mode of action which will be evaluated in selected patients already on dialysis and with metastatic disease.

47

\section{Treatment patterns among patients with metastatic renal cell carcinoma receiving systemic therapies in US real-world settings between 2006 and 2017}

Madsen, Ann (Pfizer, Inc. Brooklyn, NY, United States)

Background: Treatment-specific patient characteristics and outcomes may change over time with new information and approved treatments. Real-world data analyses aimed at informing clinical decisionmaking must account for non-random treatment assignment by physicians. Treatment pattern changes in $1 \mathrm{~L} \mathrm{mRCC}$ across periods defined by approved therapies have not been described in $\mathrm{mRCC}$.

Methods: A retrospective cohort study of newly diagnosed mRCC patients initiating FDA-approved systemic treatment was conducted within the deidentified Optum Clinformatics ${ }^{\mathrm{TM}}$ administrative claims database and stratified by year of treatment initiation. Counts and proportions of patients assigned to first (1L) systemic treatment, patient characteristics and duration of therapy were determined. Significance testing was not performed. Results for current 1L SOC treatments, sunitinib and pazopanib, are highlighted.

Following pazopanib approval (2009-2011), sunitinib- and pazopanib-treated patients numerically differed in age $(62.4 \pm 9.8$ years vs. $59.7 \pm 10.7$ years; age $\geq 65,38.1 \%$ vs $35.2 \%$ ), region ( $46.7 \%$ vs $63.0 \%$ South) and complete nephrectomy within 6 months of initiating therapy $(22.7 \%$ vs. $31.5 \%)$. Gender, insurance, comorbidity index, pre-index inpatient hospital admissions (RCC and overall) were similar. During 2012-2014, the pattern persisted with the proportion of elderly (age $\geq 65,59.1 \%$ vs 
49.8\%) and Medicare-insured (51.5\% vs $41.8 \%$ ) sunitinib patients increasing. In 2015-2016, sunitinib patients had a lower average age than pazo-

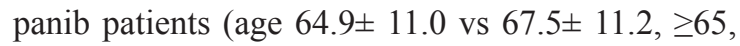
$49.7 \%$ vs $62.6 \%$ ), a higher proportion males $(74.9 \%$ vs $66.7 \%$ ), more pre-index RCC-hospitalizations (47.2\% vs 39.6\%), lower Medicare (45.7\% vs $55.4 \%)$, and similarly complete nephrectomy proportions $(34.7 \%$ vs $37.8 \%)$.

Treatment-specific duration of therapy (DoT) measures changed over time. During 2006-2008, $32.9 \%$ and $10.6 \%$ of sunitinib initiators remained on therapy at 6 and 12 months, respectively, compared with $26.0 \%$ and $8.6 \%$ in $2009-2011,30.0 \%$ and $9.7 \%$ in $2012-2014$ and $25.1 \%$ and $7.0 \%$ during 2015-2016. During 2009-2011, pazopanib-treated patients' DoT at 6 and 12 months were $35.2 \%$ and $18.5 \%$; falling to $26.2 \%$ and $12.9 \%$ in $2012-2014$ and $27.9 \%$ and $7.7 \%$ in $2015-2016$.

Conclusion: The study results demonstrate that $1 \mathrm{~L}$ mRCC baseline patient demographics and treatment patterns have evolved over time given the rapidly changing treatment environment that saw three targeted mRCC therapies in 2007 to ten available in mid-2017. Specific reasons and implications of why baseline patient characteristics have changed over time requires future research; however, given these results, real-world data studies in $\mathrm{mRCC}$ should evaluate the need for matching patient characteristics and accounting for temporal impact of when the patient initiated treatment. Without these adjustments, RWD studies in mRCC may be confounded and lead to biased results.
48

\section{Updated Results From a Phase I Study of Nivolumab in Combination With Ipilimumab in Metastatic Renal Cell Carcinoma: The CheckMate 016 Study}

Plimack, Elizabeth R. (Fox Chase Cancer Center, Philadelphia, PA, United States); Bauer, Todd M (Sarah Cannon Research Institute/ Tennessee Oncology, PLLC, Nashville, United States); Pal, Sumanta (City of Hope Comprehensive Cancer Center, Duarte, United States); Carducci, Michael; Hammers, Hans J. (Johns Hopkins Sidney Kimmel Comprehensive Cancer Center, Baltimore, United States); Rini, Brian (Cleveland Clinic Taussig Cancer Institute, Cleveland, United States); Voss, Martin (Memorial Sloan Kettering Cancer Center, New York, United States); Ernstoff, Marc S.; Lewis, Lionel D. (The Geisel School of Medicine and The Norris Cotton Cancer Center at Dartmouth-Hitchcock Medical Center, Lebanon, United States); McDermott, David F. (Beth Israel Deaconess Medical Center, Dana-Farber/Harvard Cancer Center, Boston, United States); Sharma, Padmanee (MD Anderson Cancer Center, University of Texas, Houston, United States); Razak, Albiruni (Princess Margaret Cancer Centre, Toronto, Canada); Kollmannsberger, Christian (British Columbia Cancer Agency, Vancouver, Canada); Heng, Daniel (Tom Baker Cancer Center, University of Calgary, Calgary, Canada); Spratlin, Jennifer (Cross Cancer Institute, University of Alberta, Edmonton, Canada); Berghorn, Elmer; Yang, Lingfeng (Bristol-Myers Squibb, Princeton, United States); Amin, Asim (Levine Cancer Institute, Carolinas HealthCare System, Charlotte, United States)

Background: Combination immune checkpoint inhibitor regimens have demonstrated enhanced anti-

\section{Results:}

\begin{tabular}{lllll}
\hline & $2006-2008$ & $2009-2011$ & $2012-2014$ & 2015-Sept 2016 \\
\hline $\begin{array}{l}\text { mRCC patients } \\
\text { initiating 1L therapy }\end{array}$ & 737 & 790 & 915 & 635 \\
Rank 1 & $\begin{array}{l}\text { Sunitinib } \\
(\mathrm{n}=395,53.6 \%)\end{array}$ & $\begin{array}{l}\text { Sunitinib } \\
(\mathrm{n}=362,45.8 \%)\end{array}$ & $\begin{array}{l}\text { Sunitinib } \\
(\mathrm{n}=330,36.1 \%)\end{array}$ & $\begin{array}{l}\text { Pazopanib } \\
(\mathrm{n}=222,35.0 \%)\end{array}$ \\
Rank 2 & $\begin{array}{l}\text { Sorafenib } \\
(\mathrm{n}=242,32.8 \%)\end{array}$ & $\begin{array}{l}\text { Temsirolimus } \\
(\mathrm{n}=130,16.5 \%)\end{array}$ & $\begin{array}{l}\text { Pazopanib } \\
(\mathrm{n}=263,28.7 \%)\end{array}$ & $\begin{array}{l}\text { Sunitinib } \\
(\mathrm{n}=199,31.3 \%)\end{array}$ \\
Rank 3 & $\begin{array}{l}\text { Bevacizumab } \\
(\mathrm{n}=49,6.6 \%)\end{array}$ & $\begin{array}{l}\text { Bevacizumab } \\
(\mathrm{n}=102,12.9 \%)\end{array}$ & $\begin{array}{l}\text { Temsirolimus } \\
(\mathrm{n}=116,12.7 \%)\end{array}$ & $\begin{array}{l}\text { Bevacizumab } \\
(\mathrm{n}=82,12.9 \%)\end{array}$ \\
Rank 4 & $\begin{array}{l}\text { Interferon-alfa-2B } \\
(\mathrm{n}=26,3.5 \%)\end{array}$ & $\begin{array}{l}\text { Sorafenib }(\mathrm{n}=84, \\
10.6 \%)\end{array}$ & $\begin{array}{l}\text { Bevacizumab } \\
(\mathrm{n}=103,11.3 \%)\end{array}$ & $\begin{array}{l}\text { Temsirolimus } \\
(\mathrm{n}=41,6.5 \%)\end{array}$ \\
Rank 5 & $\begin{array}{l}\text { Temsirolimus } \\
(\mathrm{n}=22,3.0 \%)\end{array}$ & $\begin{array}{l}\text { Pazopanib } \\
(\mathrm{n}=54,6.8 \%)\end{array}$ & $\begin{array}{l}\text { Everolimus } \\
(\mathrm{n}=43,4.7 \%)\end{array}$ & $\begin{array}{l}\mathrm{Nivolumab} \\
(\mathrm{n}=36,5.7 \%)\end{array}$ \\
Other & $\mathrm{N}=3,0.4 \%$ & $\mathrm{~N}=58,7.3 \%$ & $\mathrm{~N}=60,6.6 \%$ & $\mathrm{~N}=55,8.7 \%$ \\
\hline
\end{tabular}


tumor activity compared with monotherapy in multiple tumor types. Two combination regimens with low or high doses of nivolumab and ipilimumab (nivolumab $3 \mathrm{mg} / \mathrm{kg}+$ ipilimumab $1 \mathrm{mg} / \mathrm{kg}$ [N3I1 arm], nivolumab $1 \mathrm{mg} / \mathrm{kg}+$ ipilimumab $3 \mathrm{mg} / \mathrm{kg}$ [N1I3 arm]) recently demonstrated efficacy and safety in the open-label, parallel-cohort, dose-escalation, phase I CheckMate 016 study in patients with metastatic renal cell carcinoma (mRCC) (Hammers et al. JCO 2017). Here we present updated safety and efficacy results in these two arms with extended follow-up.

Methods: Patients with $\mathrm{mRCC}$ received intravenous N3I1, N1I3, every 3 weeks for four doses, followed by nivolumab monotherapy $3 \mathrm{mg} / \mathrm{kg}$ every 2 weeks until progression or toxicity. Updates to key endpoints included safety/tolerability (primary) and preliminary efficacy (secondary/exploratory).

Results: Forty-seven patients were each assigned to the N3I1 and N1I3 arms. Median follow-up was 37.7 (N3I1) and 36.0 (N1I3) months, respectively. Patients in the N3I1 and N1I3 arms received a median of 10.0 and 7.0 doses of nivolumab, respectively. Grade 3-4 treatment-related adverse events (AEs) were reported in 43\% (N3I1) and 64\% (N1I3) of patients; The most common grade 3-4 treatment-related AEs with possible immune-mediated etiology in the N3I1 and N1I3 arms were gastrointestinal (4\% and $23 \%$ ) and hepatic (6\% and 21\%). Any-grade treatment-related AEs leading to discontinuation occurred in 11\% (N3I1) and 26\% (N1I3) of patients. Antitumor efficacy is summarized in the table. The median duration of response (DOR) was 105.0

\begin{tabular}{lll}
\hline & $\begin{array}{l}\text { N3I1 } \\
\mathbf{n = 4 7}\end{array}$ & $\begin{array}{l}\text { N1I3 } \\
\mathbf{n}=47\end{array}$ \\
\cline { 2 - 3 } $\begin{array}{l}\text { Confirmed ORR, n (\%) } \\
\text { 95\% CI }\end{array}$ & $\begin{array}{l}17(36.2) \\
22.7-51.5\end{array}$ & $\begin{array}{l}19(40.4) \\
26.4-55.7\end{array}$ \\
\hline $\begin{array}{l}\text { BOR, n (\%) } \\
\text { Complete response }\end{array}$ & $5(10.6)$ & $1(2.1)$ \\
$\begin{array}{l}\text { Partial response } \\
\text { Stable disease }\end{array}$ & $12(25.5)$ & $18(38.3)$ \\
$\begin{array}{l}\text { Progressive disease } \\
\text { Unable to determine }\end{array}$ & $9(19.1)$ & $19(40.4)$ \\
$\begin{array}{l}\text { Median DOR, weeks } \\
\text { (95\% CI) }\end{array}$ & $105.0(50.7-\mathrm{NR})$ & $\mathbf{7}(14.9)$ \\
$\begin{array}{l}\text { Median PFS, months } \\
\text { (95\% CI) }\end{array}$ & $7.0(3.1-10.9)$ & $9.4(6.6-20.0)$ \\
$\begin{array}{l}\text { Median OS, months } \\
\text { (95\% CI) }\end{array}$ & NR (26.7-NR) & NR (30.0-NR) \\
\hline
\end{tabular}

BOR, best overall response; CI, confidence interval, PFS, progression-free survival; NR, not reached weeks and 79.4 weeks in the N3I1 and N1I3 arms, respectively. Median overall survival (OS) was not reached in either arm.

Conclusions: This study was the first to investigate the combination of two checkpoint inhibitors for the treatment of mRCC. Long-term follow-up continues to support the safety and promising efficacy of nivolumab plus ipilimumab combination therapy in mRCC. While both the N3I1 and N1I3 arms had high objective response rate (ORR) and durable responses with promising OS, the better safety profile observed with the lower ipilimumab dose regimen support the phase III clinical development of N3I1 in CheckMate 214 (NCT02231749).

49

\section{Updated Results From a Phase I Study of Nivolumab in Combination With Sunitinib or Pazopanib in Metastatic Renal Cell Carcinoma: The CheckMate 016 Study}
Amin, Asim (Levine Cancer Institute, Carolinas
HealthCare System, Charlotte, NC, United States);
Plimack, Elizabeth R (Fox Chase Cancer Center,
Philadelphia, United States); Lewis, Lionel D; Ernstoff,
Marc S (The Geisel School of Medicine and The Norris
Cotton Cancer Center at Dartmouth-Hitchcock Medical
Center, Lebanon, United States); Bauer, Todd M (Sarah
Cannon Research Institute/Tennessee Oncology, PLLC,
Nashville, United States); McDermott, David F. (Beth
Israel Deaconess Medical Center, Dana-Farber/Harvard
Cancer Center, Boston, United States); Carducci,
Michael (Johns Hopkins Sidney Kimmel Comprehensive
Cancer Center, Baltimore, United States);
Kollmannsberger, Christian (British Columbia Cancer
Agency, Vancouver, Canada); Rini, Brian (Cleveland
Clinic Taussig Cancer Institute, Cleveland, United
States); Heng, Daniel (Tom Baker Cancer Center,
University of Calgary, Calgary, Canada); Knox, Jennifer (Princess Margaret Cancer Centre, Toronto, Canada);
Voss, Martin (Memorial Sloan Kettering Cancer Center,
New York, United States); Spratlin, Jennifer (Cross
Cancer Institute, University of Alberta, Edmonton, Canada); Berghorn, Elmer; Yang, Lingfeng (Bristol- Myers Squibb, Princeton, United States); Hammers, Hans J. (Sidney Kimmel Comprehensive Cancer Center, Johns Hopkins, Baltimore, United States)

Background: Preliminary results from the phase I, open-label, parallel-cohort, dose-escalation CheckMate 016 study combining nivolumab, a fully 
human IgG4 programmed death-1 (PD-1) immune checkpoint inhibitor antibody, with the tyrosine kinase inhibitors (TKIs) sunitinib or pazopanib for patients with metastatic renal cell carcinoma (mRCC), have shown encouraging clinical activity, albeit with substantial toxicity (Amin et al. ESMO 2014). Here we present updated safety and efficacy results with extended follow-up.

Methods: Patients with $\mathrm{mRCC}$ received nivolumab plus sunitinib (50 mg, 4 weeks on/2 weeks off; arm $\mathrm{N}+\mathrm{S})$ or pazopanib $(800 \mathrm{mg} /$ day; arm $\mathrm{N}+\mathrm{P})$ until progression/unacceptable toxicity. The nivolumab starting dose was $2 \mathrm{mg} / \mathrm{kg}$ every 3 weeks, with planned escalation to $5 \mathrm{mg} / \mathrm{kg}$ every 3 weeks. Arm $\mathrm{N}+\mathrm{S}$ advanced to expansion; arm $\mathrm{N}+\mathrm{P}$ was closed due to dose-limiting toxicities. Primary endpoints were safety/tolerability; the secondary endpoint was preliminary antitumor activity.

Results: Arm N+S enrolled 33 patients, 19 of whom were treatment-naïve, and arm $\mathrm{N}+\mathrm{P}$ enrolled 20 patients, all of whom had $\geq 1$ prior systemic therapy. Median follow-up was 50.0 (arm N+S) and 27.1 $(\operatorname{arm} \mathrm{N}+\mathrm{P})$ months. Median duration of therapy $(\mathrm{N}+\mathrm{S})$ was 45.1 weeks for nivolumab and 28 weeks for sunitinib. Median duration of therapy $(\mathrm{N}+\mathrm{P})$ was 15.1 weeks for nivolumab and 13.9 weeks for pazopanib. The most common any-grade drugrelated adverse events (AEs) were fatigue, diarrhea, and nausea. Generally, treatment with $\mathrm{N}+\mathrm{S}$ or $\mathrm{N}+\mathrm{P}$ resulted in greater frequencies of AEs, serious AEs, AEs leading to discontinuation, and select AEs than previously observed with either agent alone (see Table). Immune-modulating medication was used in $18 / 33(55 \%)$ patients in arm $\mathrm{N}+\mathrm{S}$ and $12 / 20(60 \%)$ of patients in arm $\mathrm{N}+\mathrm{P}$. Investigator-assessed objective response rates were $55 \%(18 / 33)$ in arm $\mathrm{N}+\mathrm{S}$, with two complete responses (6.1\%), and $45 \%$ $(9 / 20)$ in arm $\mathrm{N}+\mathrm{P}$, with no complete responses. Median (95\% confidence interval [CI]) duration of response was 60.2 (37.1-not reached) weeks in arm $\mathrm{N}+\mathrm{S}$ and 30.1 (12.1-174.1) weeks in arm $\mathrm{N}+\mathrm{P}$. Median (95\% CI) progression-free survival was 12.7 (11.0-16.7) months in arm N+S and 7.2 (2.8-11.1) months in arm $\mathrm{N}+\mathrm{P}$. Overall survival will be reported.

Conclusions: The addition of sunitinib or pazopanib to nivolumab showed encouraging antitumor activity and progression-free survival in pretreated and frontline patients with $\mathrm{mRCC}$, however, a higher incidence of high-grade toxicities was observed with the combinations compared with nivolumab, suni- tinib, or pazopanib monotherapy. Combination strategies of nivolumab with ipilimumab and other TKIs are currently under investigation.

50

\section{Validation of the Preoperative Nomogram Predicting 12-Year Probability of Metastatic Renal Cancer}

Ghanaat, Mazyar (Memorial Sloan Kettering Cancer Center, New York, NY, United States); Duzgal, Cihan (Body Imaging Service, Department of Radiology, Memorial Sloan Kettering Cancer Center, New York, United States); Blum, Kyle; Kashan, Mahyar; Sanchez, Alejandro; DiNatale, Renzo; Becerra, Maria; Ranasinghe, Buddima; Benfante, Nicole (Urology Service at the Department of Surgery, Memorial Sloan Kettering Cancer Center, New York, United States); Coleman, Jonathan (Urology Service at the Department of Surgery, Memorial Sloan Kettering Cancer Center, New York, United States); Kattan, Michael W. (Department of Quantitative Health Sciences, Cleveland Clinic, Cleveland, United States); Akin, Oguz (Body Imaging Service, Department of Radiology, Memorial Sloan Kettering Cancer Center, New York, United States); Ostrovnaya, Irina (Department of Epidemiology and Biostatistics, Memorial Sloan Kettering Cancer Center, New York, United States); Hakimi, A. Ari (Urology Service at the Department of Surgery, Memorial Sloan Kettering Cancer Center, New York, United States)

Introduction and objectives: We previously published a predictive model to determine the preoperative risk of metastatic recurrence in localized renal cell carcinoma. We sought to validate this initial nomogram and interrogate the additive value of somatic mutations in a subcohort with available genomic data.

Methods: We conducted a retrospective review of all non metastatic patients at a single tertiary referral center from 2004-2011 who underwent a surgical extirpation for a renal mass $(\mathrm{n}=2391)$. Mutations in VHL, PBRM1, SETD2, BAP1, KDM5C for those patients who had genomic analysis by previously described MSK IMPACT were recorded. Nomogram for 12-year metastasis free survival published by Raj et al in 2008 was validated using Kaplan-Meier estimates. Associations between covariates and time to metastasis were calculated by Cox regression.

Results: An initial cohort of 281 patients was available for analysis. Median age at time of surgery was 


\begin{tabular}{lccccc}
\hline & \multicolumn{2}{c}{ Arm N+S } & & \multicolumn{2}{c}{ Arm N+P } \\
& \multicolumn{2}{c}{$\mathrm{N}=33$} & & \multicolumn{2}{c}{$\mathrm{N}=20$} \\
\cline { 2 - 3 } \cline { 5 - 6 } $\mathrm{n}(\%)$ & Any grade & Grade 3-4 & & Any grade & Grade 3-4 \\
\hline Treatment-related AEs & $33(100)$ & $27(82)$ & & $20(100)$ & $14(70)$ \\
Treatment-related AEs leading to & $13(39)$ & $11(33)$ & & $5(25)$ & $4(20)$ \\
discontinuation & & & & & \\
Treatment-related serious AEs & $14(42)$ & $10(30)$ & & $2(10)$ & $2(10)$ \\
Select treatment-related AEs & & & & & \\
Gastrointestinal & $21(64)$ & $3(9)$ & & $12(60)$ & $4(20)$ \\
Hepatic & $15(45)$ & $8(24)$ & & $7(35)$ & $4(20)$ \\
Pulmonary & $1(3)$ & $1(3)$ & & $1(5)$ & 0 \\
Renal & $13(39)$ & $4(12)$ & & $1(5)$ & 0 \\
Skin & $26(79)$ & $2(6)$ & & $11(55)$ & 0 \\
Endocrine & $12(36)$ & 0 & & $5(25)$ & $2(10)$ \\
\hline
\end{tabular}

${ }^{\mathrm{a} A E s}$ with possible immune-mediated etiology

61.3 (24.7-84). Table 1 lists the clinical characteristics and associations to time to metastasis. There were 33 patients who developed metastatic disease on median follow-up of 9 years (Figure 1). Associations between the five preoperative characteristics and time to metastasis were similar to the original report. The linear predictor from the nomogram was highly associated with metastasis free survival $(p<0.0001)$. We split the predicted 12-year metastasis free probability into quartiles, and used them to calculate the estimated $12-$ year survival in this cohort: it was not estimable in the first quartile, and $37.5 \%, 71 \%$ and $92 \%$ in $2 \mathrm{nd}$, 3rd and 4th quartile, indicating good calibration of the original nomogram (Figure 2). KDM5C was significantly associated with metastasis-free survival and remained significant after incorporating nomogram prediction into the model $(\mathrm{p}=0.04, \mathrm{HR}=3.6,95 \%$ CI $1.05,12.4)$.

Conclusions: Univariate assessment of factors in our original model are associated with metastatic recurrence. Further statistical analysis of the complete cohort and integration of genomic data is ongoing.

Supported by the Sidney Kimmel Center for Prostate and Urologic Cancers, Ruth L. Kirschstein National Research Service Award T32CA082088 (MG\& AS)

\section{1}

Variations in treatment patterns for metastatic renal cell carcinoma (mRCC) between developing and developed countries

Bergerot, Paulo (City of Hope Comprehensive Cancer Center, Monrovia, CA, United States); Bergerot, Cristiane
(City of Hope Comprehensive Cancer Center, Monrovia, $C A$, United States); Dizman, Nazli (City of Hope Comprehensive Cancer Center, Duarte, CA, United States); Zequi, Stenio (AC Camargo Cancer Center, Sao Paulo, Brazil); Fay, Andre (Pontificia Universidade Catolica do Rio Grande do Sul, Porto Alegre, Brazil); Dara, Yash (City of Hope Comprehensive Cancer Center, Duarte, United States); Manuel, Maia; Cotta, Brendan N. (City of Hope Comprehensive Cancer Center, Duarte, $C A$, United States); Goncalves, Edna P. (Close-Up International, Sao Paulo, Brazil); Formiga, Maria N.; Tariki, Milena S. (AC Camargo Cancer Center, Sao Paulo, Brazil); Clavijo, Diego A. (Hospital Pasteur, Montevideu, Uruguay); Choueiri, Toni (Dana Farber Cancer Institute, Boston, MA, United States); Lopes, Gilberto (Sylvester Cancer Center, Miami, FL, United States); Pal, Sumanta K. (City of Hope Comprehensive Cancer Center, Duarte, CA, United States)

Background: We have previously reported patterns of care for mRCC using a large US-based retrospective chart review encompassing 1,173 patients (pts) (Pal et al Int J Urol 2017). We hypothesize that treatment patterns in developing and developed countries will have marked variation.

Methods: From Jan 2013 to Dec 2016, pts with $\mathrm{mRCC}$ receiving treatment at private or public hospitals in Brazil had receipt of systemic therapy (tx) recorded in a prospective database. Basic clinical and demographic criteria were available, as well as information to ascertain Heng risk. Trends in use of $1^{\text {st }}$-line $(1 \mathrm{~L}), 2^{\text {nd }}$-line $(2 \mathrm{~L})$ and $3^{\text {rd }}$-line $(3 \mathrm{~L})$ tx were compared to the previously referenced US-based chart review, which collected data over an overlapping timeframe. The chi-square test was used to compare treatment frequencies across cohorts.

Results: Of 4,379 pts assessed, 3,990 pts (91\%) had metastatic disease and $21 \%, 38 \%$ and $21 \%$ of pts had good, intermediate and poor risk disease, respective- 


\begin{tabular}{|c|c|c|c|}
\hline & $\mathbf{N}(\%)$ & Hazard Ratio(CI) & P-value \\
\hline \multicolumn{3}{|l|}{ Gender } & 0.014 \\
\hline Male & $196(70 \%)$ & $4.4(1.34-14.41)$ & \multirow[t]{2}{*}{0.014} \\
\hline Female & $85(30 \%)$ & 1 & \\
\hline \multicolumn{3}{|l|}{ Mode of Presentation } & \multirow[t]{5}{*}{0.058} \\
\hline Incidental & $229(81.5)$ & 1 & \\
\hline Local & $48(17.1)$ & $1.93(0.89-4.18)$ & \\
\hline Systemic & $2(0.7)$ & $6.75(0.91-50.22)$ & \\
\hline Not available & $2(0.7)$ & NA & \\
\hline \multicolumn{3}{|l|}{ Lymphadenopathy } & \multirow[t]{3}{*}{0.062} \\
\hline No & $255(91)$ & 1 & \\
\hline Yes & $26(9)$ & $2.32(0.96-5.63)$ & \\
\hline Necrosis & & & 0.01 \\
\hline No & $147(52)$ & 1 & \\
\hline Yes & $134(48)$ & $2.67(1.27-5.6)$ & \\
\hline Tumor size (range) & $3.9 \mathrm{~cm}(1-18.7)$ & $1.21(1.12-1.3)$ & $<0.001$ \\
\hline \multicolumn{4}{|c|}{ Genomic Analysis $n=149$} \\
\hline $\mathrm{VHL}$ & $71(25.3)$ & $1.26(0.56-2.86)$ & 0.578 \\
\hline PBRM1 & $45(16)$ & $1.22(0.52-2.89)$ & 0.646 \\
\hline SETD2 & $15(5.3)$ & $2.57(0.95-6.93)$ & 0.062 \\
\hline BAP1 & $5(1.8)$ & NA & NA \\
\hline KDM5C & $7(2.5)$ & $3.53(1.04-12.02)$ & 0.044 \\
\hline Not available & $132(47)$ & NA & NA \\
\hline
\end{tabular}

Figure 1. Metastasis free survival

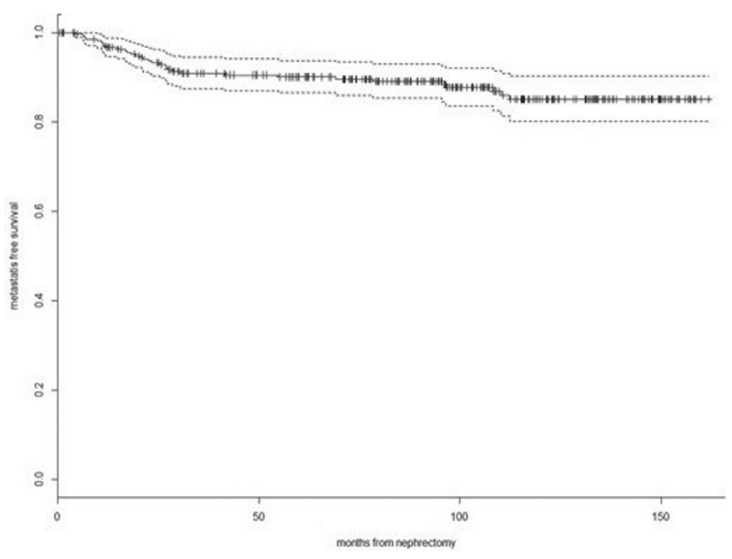

Figure 2. Metastasis free survival stratified by the quartiles of predicted 12-year metastasis free probability

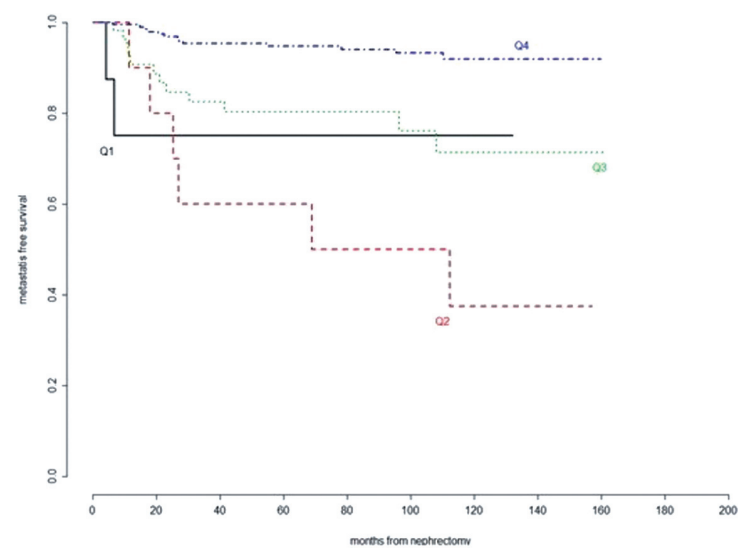

ly. While 3,149 pts $(78 \%)$ received $1 \mathrm{~L}$ therapy, only $641 \mathrm{pts}(20 \%)$ and $152 \mathrm{pts}(5 \%)$ received 2L and 3L therapy, respectively. In comparison to the US chart review $(\mathrm{n}=1,123)$, there was lower use of sunitinib in Brazil (44.5\% vs $74.1 \%, \mathrm{P}=.05)$ and higher use of pazopanib $(21.7 \%$ vs $13.2 \%, \mathrm{P}=.08)$. In the $2 \mathrm{~L}$ setting, patients in the current cohort had a higher use of everolimus versus patients in the US $(37.3 \%$ vs $27.8 \%, \mathrm{P}=.05$ ). Pazopanib, sunitinib and sorafenib were also frequently used in this setting. In the $3 \mathrm{~L}$ setting, tx was variable with sorafenib and pazopanib representing the most commonly utilized regimens. Substantial tx heterogeneity was noted, with a total of 56 distinct regimens recorded including cytotoxic therapy (e.g., bleomycin, vinflunine and adriamycin gemcitabine).

Conclusions: Relative to a high resource setting, marked attrition is noted between $1 \mathrm{~L}$ and $2 \mathrm{~L}$ therapy in a representative developing country. Patterns of care vary as well for $1 \mathrm{~L}$ and $2 \mathrm{~L}$ therapy, and tx heterogeneity with many antiquated regimens may reflect lack of access to targeted tx or potentially addressable gaps in physician education. 


\section{2}

\section{Active Surveillance for von Hippel- Lindau-Related Renal Tumors using Size-Based Risk Stratification: Long- term Results}

Mark W. Ball (National Cancer Institute, Baltimore, MD, United States); Julie An; James Peterson; Adam R. Metwalli; Maria J. Merino; Ramaprasad Srinivasan; W. Marston Linehan (National Cancer Institute, Baltimore, MD, United States)

Introduction: Renal cell carcinoma (RCC) develops in $25-60 \%$ of patients von Hippel-Lindau (VHL), which is characterized by germline mutations in the $V H L$ gene. Our institution practice has been to perform active surveillance for renal lesions less than $3 \mathrm{~cm}$ and surgical resection for lesions greater than $3 \mathrm{~cm}$, based on early observations of low metastatic potential of small lesions. However, patients who are referred with larger tumors or who are lost to follow-up may not be managed exclusively by this guideline. We sought to evaluate the oncologic efficacy of the $3 \mathrm{~cm}$ size threshold with in a large cohort with long-term follow-up.

Methods: From a prospective registry of 764 patients with VHL, a subset of patients with solid renal masses was identified. The diameter of the largest solid tumor, length of follow-up, and development of metastatic disease was abstracted from imaging reports and the medical record. Patients were further subdivided into those who were managed exclusively by the $3 \mathrm{~cm}$ threshold and those who were not. The proportion of patients who developed metastatic disease at size thresholds beyond $3 \mathrm{~cm}$ was assessed in
$1 \mathrm{~cm}$ increments. Follow-up was defined as the interval from initial screening at institution to last clinic visit or progression. Metastasis-free survival (MFS) was defined as the interval from initial screening to development of distant metastatic disease.

Results: A total of 440 patients (57.5\%) developed solid kidney tumors. Of these 417 (94.7\%) had prior imaging reports available. Median follow-up was 103 months. Metastatic disease developed in 42 patients $(10.1 \%)$. No patients developed metastatic disease when the size of their largest tumor was $<3$ $\mathrm{cm}$. Table 1 lists the proportion of patients who developed metastases by size of their largest tumor. MFS for patients managed with the $3 \mathrm{~cm}$ threshold was significantly longer compared to those who were not $(\mathrm{p}=0.007)$. The 5, 10 and 20-year MFS for patients who were not managed by the $3 \mathrm{~cm}$ threshold was $95.7 \%, 91.1 \%$, and $69.5 \%$, respectively.

Conclusion: In a large cohort of patients with VHL, adherence to the $3 \mathrm{~cm}$ guideline was associated with superior MFS compared to those who were not. We advocate the use of this guideline in conjunction with other patient characteristics and surgical judgement.

Table 1

\begin{tabular}{cc}
\hline Tumor Size & n Mets/n pts (\%) \\
\hline$<3 \mathrm{~cm}$ & $0 / 148(0 \%)$ \\
$3-4 \mathrm{~cm}$ & $4 / 139(2.9 \%)$ \\
$4-5 \mathrm{~cm}$ & $8 / 62(12.9 \%)$ \\
$5-6 \mathrm{~cm}$ & $7 / 27(25.9 \%)$ \\
$6-7 \mathrm{~cm}$ & $6 / 12(50 \%)$ \\
$7-8 \mathrm{~cm}$ & $7 / 13(53.8 \%)$ \\
$8-9 \mathrm{~cm}$ & $2 / 5(40 \%)$ \\
$9-10 \mathrm{~cm}$ & $3 / 4(75 \%)$ \\
$>10$ & $5 / 6(83.3 \%)$ \\
\hline
\end{tabular}

